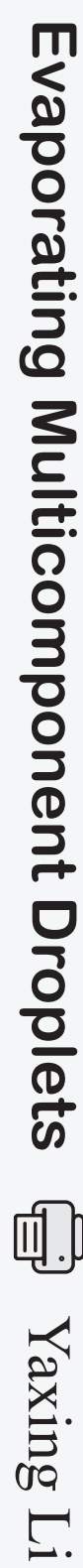

Evaporating Multicomponent Droplets

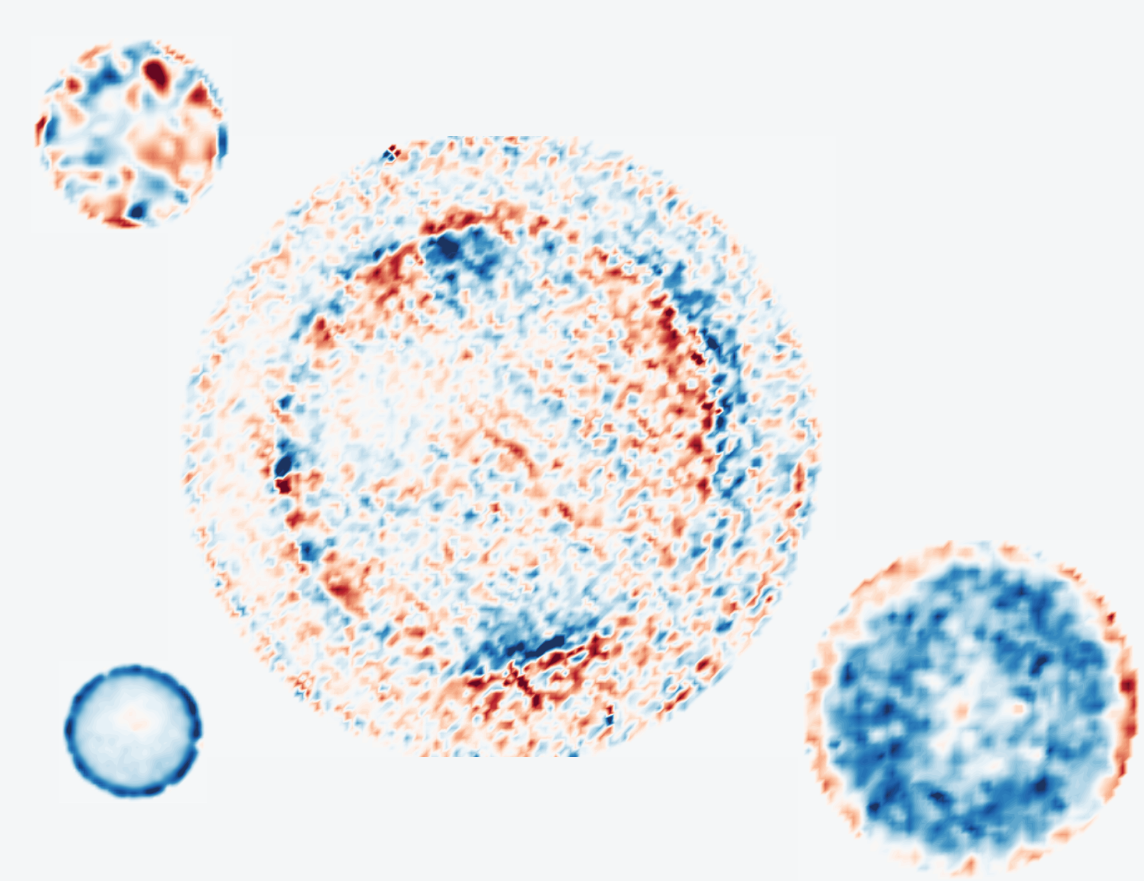

Yaxing Li 
Evaporating multicomponent droplets

Yaxing Li 


\section{Graduation committee:}

Prof. dr. J. L. Herek (chairman)

Prof. dr. ret. nat. D. Lohse (promotor)

Prof. dr. M. Versluis (promotor)
University of Twente University of Twente University of Twente
Prof. dr. U. Thiele

Prof. dr. ir. H. Wijshoff

Prof. dr. G. Rijnders

Dr. S. Kooij

Dr. M. van der Linden
Westfälische Wilhelms-Universität Münster Eindhoven University of Technology University of Twente University of Twente Canon Production Printing

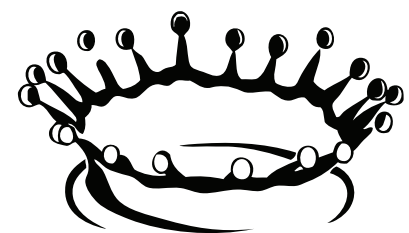

Physics of Fluids

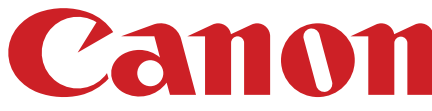

MAX-PLANCK-GESELLSCHAFT

The work in this thesis was carried out at the Physics of Fluids group of the Faculty of Science and Technology of the University of Twente.

Dutch title:

Verdampende druppels bestaande uit meerdere componenten

Publisher:

Yaxing Li, Physics of Fluids, University of Twente, P.O. Box 217, 7500 AE Enschede, The Netherlands

Copyright (0) Yaxing Li, Enschede, The Netherlands 2020

All rights reserved. No part of this work may be reproduced or transmitted for commercial purposes, in any form or by any means, electronic or mechanical, including photocopying and recording, or by any information storage or retrieval system, except as expressly permitted by the publisher.

ISBN: 978-90-365-5004-8

DOI: $10.3990 / 1.9789036550048$ 


\section{EVAPORATING MULTICOMPONENT DROPLETS}

\section{DISSERTATION}

to obtain

the degree of doctor at the University of Twente, on the authority of the rector magnificus,

Prof. Dr. T. T. M. Palstra,

on account of the decision of the graduation committee,

to be publicly defended

on Friday, June $26^{\text {th }}, 2020$ at $16: 45$

by

Yaxing Li

Born on December $22^{\text {th }}, 1991$

in Anhui, China 
This dissertation has been approved by the promotors:

Prof. dr. ret. nat. D. Lohse

and

Prof. dr. M. Versluis 


\section{Contents}

$\begin{array}{ll}\text { Introduction } & 1\end{array}$

1 Evaporation-triggered segregation of sessile binary droplets 11

1.1 Introduction . . . . . . . . . . . . . . . . . 13

1.2 Experimental results . . . . . . . . . . . . . . . . . . 14

1.3 Numerical simulations . . . . . . . . . . . . . . . . . . . 18

1.4 Conclusions and discussions . . . . . . . . . . . . . . . 21

1.5 Supplemental Materials . . . . . . . . . . . . . . 22

2 Gravitational effect in evaporating binary microdroplets $\quad 27$

2.1 Introduction . . . . . . . . . . . . . . . . . 29

2.2 MicroParticle Image Velocimetry . . . . . . . . . . . . . . 30

2.3 Theoretical analysis . . . . . . . . . . . . . . 33

2.4 Numerical simulations . . . . . . . . . . . . . . . . . 36

2.5 Conclusions . . . . . . . . . . . . . . . . . . . 39

2.6 Supplemental Materials . . . . . . . . . . . . . . . . 39

3 Rayleigh-Taylor instability by segregation in an evaporating multicomponent microdroplet 43

3.1 Introduction . . . . . . . . . . . . . . . . 46

3.2 Experimental methods . . . . . . . . . . . . . . . 47

3.3 Rayleigh-Taylor instability arising from segregation . . . . . . . 49

3.4 Evaporation process and its quantitative understanding . . . 57

3.5 Summary and outlook . . . . . . . . . . . . . . 66

3.6 Appendix . . . . . . . . . . . . . . . 67

4 Evaporation-induced crystallization of surfactants in sessile $\begin{array}{lr}\text { multicomponent droplets } & 69\end{array}$

4.1 Introduction . . . . . . . . . . . . . . . . . . 71 
4.2 Experimental Methods . . . . . . . . . . . . . . . . . . . 72

4.3 Experimental results . . . . . . . . . . . . . . . . . . 74

4.4 Theoretical analysis . . . . . . . . . . . . . . 76

4.5 Conclusions and outlook . . . . . . . . . . . . . . . 82

4.6 Supplemental Materials . . . . . . . . . . . . . . . 82

5 Evaporating droplets on lubricated surfaces: Suppression of $\begin{array}{ll}\text { the coffee-stain effect } & 87\end{array}$

5.1 Introduction . . . . . . . . . . . . . . . . . . 90

5.2 Experimental results . . . . . . . . . . . . . . . . . . . 92

5.3 Hydrodynamic model . . . . . . . . . . . . . . . . . . . 98

5.4 Numerical investigation . . . . . . . . . . . . . . . . . 99

5.5 Conclusions . . . . . . . . . . . . . . . . . . 100

5.6 Experimental Methods . . . . . . . . . . . . . . . . . . . . 101

5.7 Numerical Model . . . . . . . . . . . . . . . . . . . . . . . . . . 101

5.8 Supplemental Materials . . . . . . . . . . . . . . . . 102

$\begin{array}{lc}\text { Conclusions and Outlook } & 113\end{array}$

$\begin{array}{lr}\text { References } & 119\end{array}$

$\begin{array}{ll}\text { Summary } & 135\end{array}$

$\begin{array}{lr}\text { Samenvatting } & 139\end{array}$

$\begin{array}{ll}\text { Scientific output } & 143\end{array}$

$\begin{array}{ll}\text { Acknowledgements } & 147\end{array}$

$\begin{array}{ll}\text { About the author } & 151\end{array}$ 


\section{Introduction}

Evaporation is a ubiquitous phenomenon in our planet - it is a type of vaporization that occurs on the surface of a liquid as it transitions to the gas phase. As one of the most common forms of liquids, droplets and their evaporation are omnipresent in nature and technology, be it a rain droplet on a lotus leaf; an inkjet droplet on a paper; a suspension droplet on an uncoated substrate; or a cooling droplet on a hot surface (see examples in Fig. 1). Over a century ago, it was already discovered that the diffusion of vapor in the surrounding gas is the mechanism that limits the evaporation rate of a droplet. Yet, it still remains one of the most challenging topics for scientists and engineerings.

\section{Diffusion-limited process}

\section{Single-component droplets}

In 1910, Harry W. Morse studied the evaporation of a small sphere of iodine in quiescent air [1]. He placed the spheres of about one millimeter diameter on the flat surface of a microbalance and weighed them at intervals until they disappeared. The experiments surprisingly indicated that the rate of the mass loss was proportional to the radius of the sphere instead of to its surface. A few years later, the linear dependency on the radius was explained theoretically by Irving Langmuir who showed that the evaporation of the small sphere of iodine is determined by the rate of diffusion through the surrounding air [2]. In 1950, Epstein \& Plesset [3] gave the mathematical solution of the diffusion problem for a spherical gas bubble dissolving in a liquid-gas solution. They calculated the gas concentration field by solving the diffusion equation:

$$
\partial c / \partial t=\kappa \Delta c
$$

where $c$ is the dissolved gas concentration in the solution at a distance $r_{g}$ 

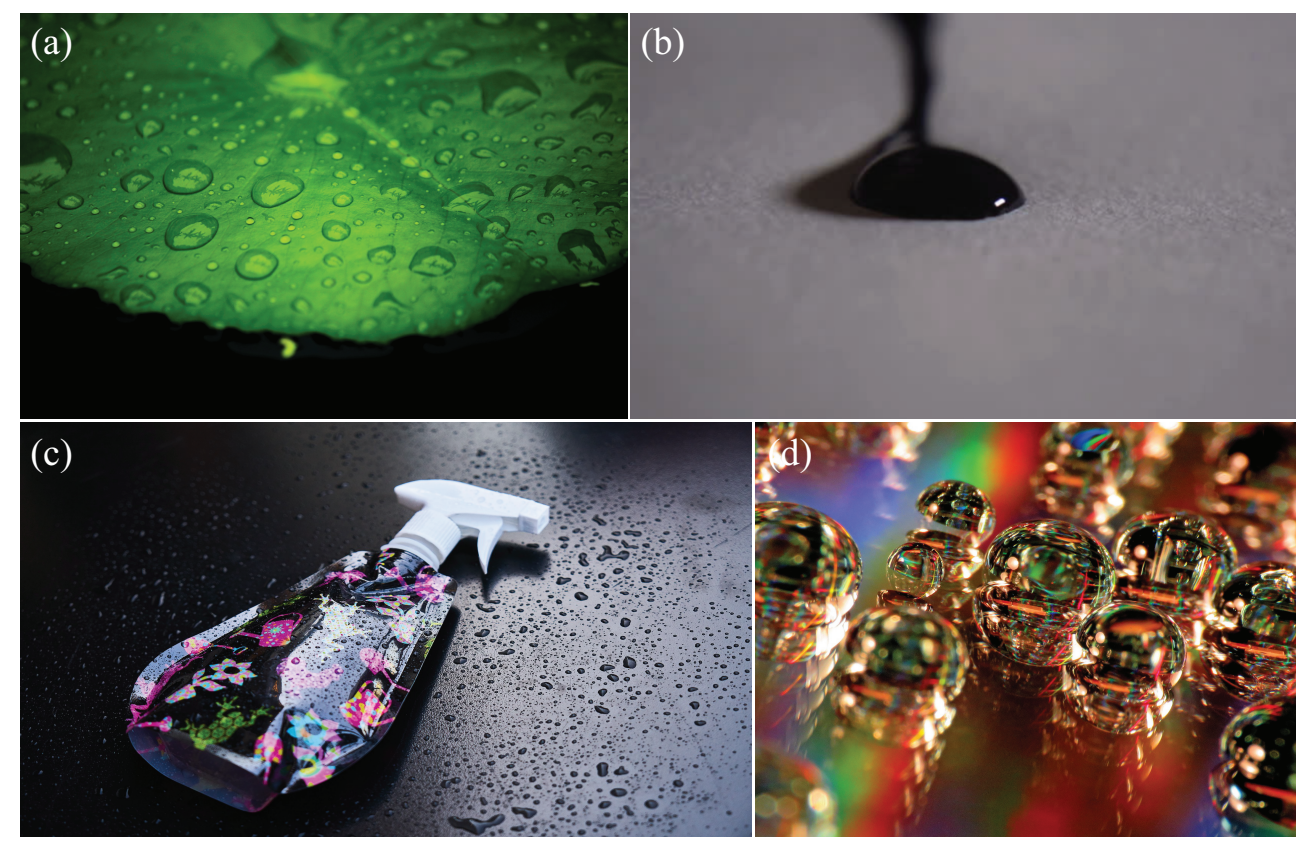

Figure 1: Examples of sessile droplets on different substrates: (a) Rain droplets on a lotus leaf; (b) A ink drop on paper; (c) Droplets sprayed on a solid surface; and (d) Droplets on a hydrophobic surface. (Source for images: a. c. d. Pixabay website; b. Shutterstock website.)

from the origin, $\kappa$ is the coefficient of diffusivity of the gas in the liquid. In a spherically symmetric solution, the following conditions are satisfied:

$$
\begin{gathered}
c(r, 0)=c_{i}, r_{g}>R_{g} ; \lim _{r_{g} \rightarrow \infty} c\left(r_{g}, t\right)=c_{i}, t>0 ; \\
c\left(R_{g}, t\right)=c_{s g}, t>0 ;
\end{gathered}
$$

where $R_{g}$ is the bubble radius, $c_{i}$ is the initial gas concentration in the surrounding liquid and $c_{s g}$ is the saturated gas concentration on the gas-liquid interface. By solving the diffusion equation together with these boundary conditions, the concentration gradient at $r_{g}=R_{g}$ can be obtained:

$$
\left(\frac{\partial c}{\partial r_{g}}\right)_{R_{g}}=\left(c_{s g}-c_{i}\right)\left[\frac{1}{R_{g}}+\frac{1}{(\pi \kappa t)^{\frac{1}{2}}}\right] .
$$


Thus, the mass flux of the gas that dissolves into the liquid has the value

$$
\dot{m}=-4 \pi R_{g}^{2} \kappa\left(\frac{\partial c}{\partial r_{g}}\right)_{R_{g}}=4 \pi R_{g}^{2} \kappa\left(c_{s g}-c_{i}\right)\left[\frac{1}{R_{g}}+\frac{1}{(\pi \kappa t)^{\frac{1}{2}}}\right] .
$$

However, different from the gas dissolution, the evaporation of droplets in air is treated to be a quasi-steady process [4] by arguing that the characteristic diffusion time $t_{d}=R^{2} / D$ is much smaller than the typical droplet evaporation time $t_{f}=\rho_{l} /\left(c_{s}-c_{\infty}\right) t_{d}$, with $D$ the diffusion coefficient, $c_{s}$ is the saturated vapor concentration, $c_{\infty}=H c_{s}$ ( $H$ relative humidity) is the vapor concentration in the surrounding air and $\rho_{l}$ is the liquid density. In this quasi-steady case, the concentration field $c$ around the droplet becomes

$$
\nabla^{2} c=0 .
$$

Therefore, the diffusive flux on the droplet surface is given by

$$
\dot{m}=4 \pi R \kappa\left(c_{s}-c_{\infty}\right),
$$

which is proportional to the radius of the droplet instead of to its surface area. For the evaporation of a sessile droplet on the substrate, another boundary condition is added: because of the impermeability of the substrate, $\partial c / \partial z=0$ along the surface, with $z$ the normal distance from the substrate. Popov [5] obtained the analytical solution for this problem by using the solution to an equivalent problem of the electric potential around a charged lens-shaped conductor:

$$
\dot{m}=-\pi D R\left(c_{\mathrm{s}}-c_{\infty}\right) f(\theta)
$$

with

$$
f(\theta)=\frac{\sin (\theta)}{1+\cos (\theta)}+4 \int_{0}^{\infty} \frac{1+\cosh (2 \theta \varepsilon)}{\sinh (2 \pi \varepsilon)} \tanh [(\pi-\theta) \varepsilon] \mathrm{d} \varepsilon
$$

where $R, \theta$ and $D$ are the footprint radius, contact angle of the droplet and diffusion coefficient of vapor in the surrounding air, respectively.

The prediction of the evaporation rate of a sessile droplet obtained by the diffusion model (Eq. 4.1) relies on the knowledge of the evaporation mode. Picknett \& Bexon [6] identified three modes in which a sessile drop can evaporate on a flat surface (see Fig. 2); one in which the drop's footprint radius remains constant (CR mode), another one in which the contact angle is constant (CA mode) and a mixed mode, in which the drop first evaporates in the 
CR mode and switches to CA mode after it reaches the receding contact angle ("Stick-Slide" mode). Recently, a fourth mode was observed [7,8], namely "Stick-Jump" mode, in which the contact line remains pinned, but suddenly jumps to the next pinning position for each time the contact angle reaches a critical value, where it then repeats the same process. So far, a complete description of the evaporation rate of a single-component droplet drying on a flat substrate is obtained [9].

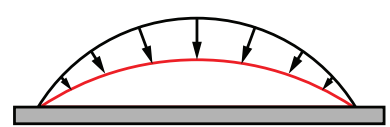

(a) CR mode

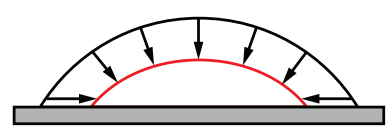

(b) CA mode

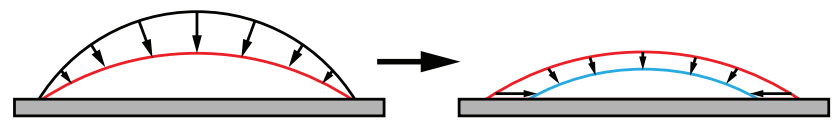

(c) Stick-slide mode

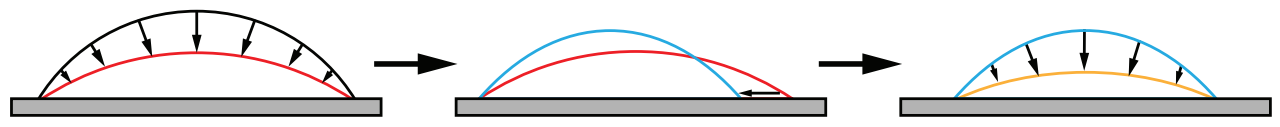

(d) Stick-jump mode

Figure 2: Schematics of four evaporation modes of a sessile droplet. (a) CR mode. The droplet evaporates with a pinned contact line. (b) CA mode. The droplet evaporates with a constant contact angle. (c) Stick-slide mode. The droplet evaporates following the CR mode then changes to the CA mode when reaching a critical contact angle. (d) Stick-jump mode. The droplet first evaporates in a $\mathrm{CR}$ mode, when it reaches the critical angle, the contact radius jumps to a smaller value due to a sudden depinning of contact line on one side. The mass conservation at the short time interval of jumping yields an increasing of the droplet height and contact angle. After the jump, the droplet again evaporates in a CR mode until the next jump.

\section{Multi-component droplets}

The essential difference between a pure liquid and a mixture is the vaporliquid equilibrium. The existence of different species in the mixture lower the saturated vapor concentration at the air-liquid interface of each component $i$ as compared to that in a pure system. Raoult's law [10] is used to calculate the air-liquid equilibrium: $c_{i, \mathrm{~s}}=X_{i} c_{i, \mathrm{~s}}^{0}$, where $X_{i}$ is the mole fraction of component 
$i$ on the interface and $c_{i, \mathrm{~s}}^{0}$ is the saturated vapor concentration of the pure liquid $i$. However, Raoult's law only relies on an ideal solution and ignores any interaction between the components. To overcome this limitation, a so-called activity coefficient $\Gamma_{\mathrm{i}}$ for each component is introduced [11]: $c_{\mathrm{i}, \mathrm{s}}=\Gamma_{\mathrm{i}} X_{\mathrm{i}} c_{\mathrm{i}, \mathrm{s}}^{0}$. Therefore, a theoretical model can be obtained to express the evaporation rate for each component $i$ in a multi-component droplet:

$$
\dot{m}_{\mathrm{i}}=-\frac{\pi}{2} D_{\mathrm{i}} R\left(\Gamma_{\mathrm{i}} X_{\mathrm{i}} c_{\mathrm{i}, \mathrm{s}}^{0}-c_{\mathrm{i}, \infty}\right) f(\theta),
$$

and the total evaporation rate is given by:

$$
\dot{m}=\Sigma \dot{m}_{\mathrm{i}}
$$

where the subindex $i$ stands for the component $i$ of each variable.

\section{Evaporation-driven flows and particle depositions}

\section{Coffee-stain effect}

While the evaporation is controlled by a diffusion process which appears to be quiet and slow, the evaporation-driven flow inside a droplet can be strong and complicated. In 1997, Deegan and his coworkers studied the flow structure in an evaporating particle-laden water droplet [12]. The observation revealed that the capillary flow caused by the pinning of the contact line, carries particles towards the edge of the droplet. It is the first work explaining the underlying mechanism describing the so-called coffee-stain effect [see Fig. 3(a)], and more importantly, it brought this daily-life phenomenon into the laboratory and it keeps inspiring the scientific community. Since then, the coffee-stain effect has became paradigmatic for a whole class of problems associated with any type of colloidal suspension droplets, such as ink, blood or salty water. The main outcome of the effect directly affects particle deposition on the surface. However, in most industrial applications ranging from surface coating, particle patterning and inkjet printing, the challenge is to prevent the formation of such ring-shaped distributions in order to obtain a uniform coating. Hence, the suppression of coffee-stain effect becomes one of the key topics for the study on flow behavior in evaporating droplets. 

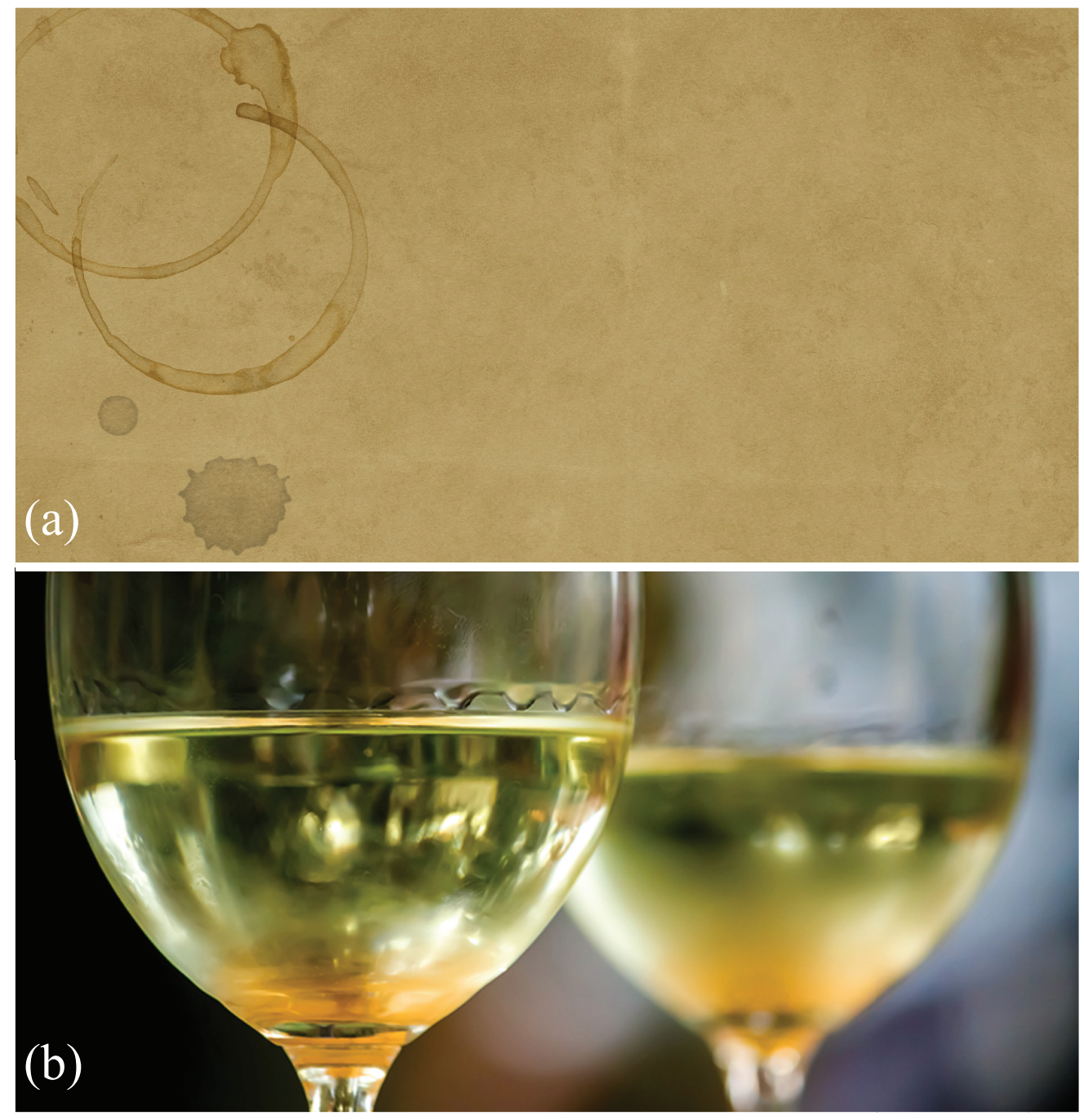

Figure 3: (a) Coffee-stains on paper (Pixabay website). (b) Tears of wines (Winecooler website).

\section{Marangoni effect}

In 1855, physicist James Thomson observed the wine dripping down the goblet's wall on his dinning table. He identified this phenomenon with a beautiful name "tears of wine" [13] [see Fig. 3(b)]. Ten years later, Italian physicist 
Carlo Marangoni published his study on this phenomenon for his doctoral dissertation and extended for more general cases [14]. The effect is thereby named after him as the "Marangoni effect". It works as follows: A liquid with a high surface tension pulls more strongly on the surrounding liquid than one with a low surface tension, the presence of surface tension gradients drives a liquid flow towards regions of high surface tension. In most droplet systems, natural convection caused by the effect of gravity becomes negligible as compared to the flow driven by surface tension gradient.

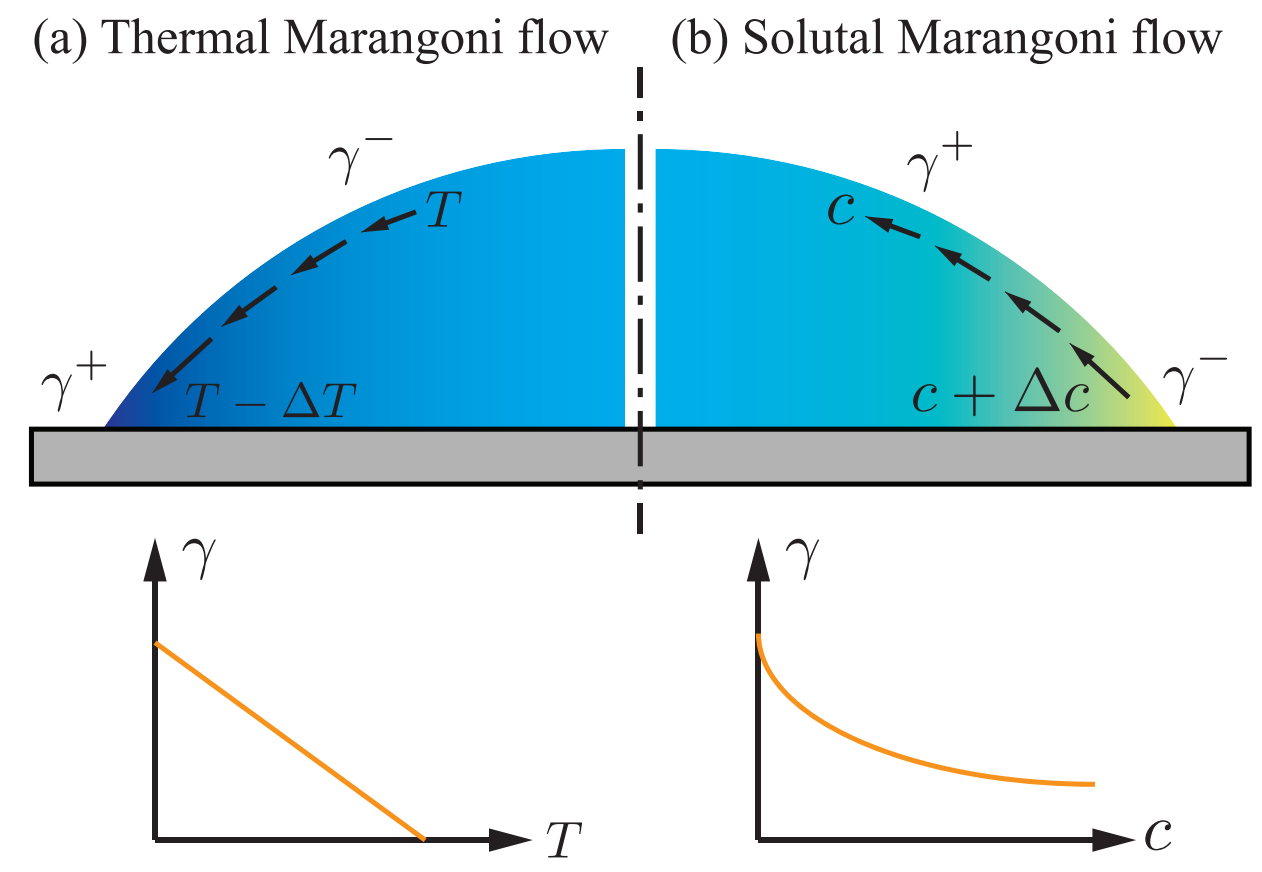

Figure 4: Schematics of two Marangoni flows. (a) Thermal Marangoni flow. (b) Solutal Marangoni flow.

The inhomogeneous evaporative flux along the droplet surface may lead to unbalanced mass and heat transfer, which causes either a concentration gradient or a temperature gradient. Hence, the resulting surface tension gradient drives a tangential flow along the surface, termed the Marangoni flow. Based on the driving source, they can be categorized into two kinds: thermal Marangoni flow and solutal Marangoni flow, see Fig. 4. The thermal Marangoni effect normally drives the flow towards the low-temperature side of the liquid as heat 
reduces the surface tension. The solutal Marangoni effect is more complicated. Here we introduce the case where the surface tension decreases with increasing solute concentration. In this case, the solutal Marangoni effect drives the flow towards the side with a lower solute concentration.

In general, the flow structure inside an evaporating droplet, either singlecomponent or multi-component, is a result of the interplay between all kinds of effects, happening at different length and time scales, which makes it complicated and difficult to predict. It is crucial to obtain insight into the flow structure because it affects the evaporation behavior in return and also determines the final depositions of particles and other suspensions.

\section{A guide through this thesis}

In this thesis, we address how selective evaporation of each component affects the evaporation behavior, flow structure and particle depositions by studying several multi-component droplet systems.

In Chapter 1, we studied the evaporation of a binary liquid system consisting of a miscible mixture of water and 1,2-hexanediol, in which only water evaporates in the laboratory conditions. We find that the preferential evaporation of water and the insufficient mixing effect by weak convection lead to a segregation of 1,2-hexanediol.

In Chapter 2, we investigated another binary liquid system by replacing 1,2hexanediol with glycerol. We find that in spite of the small droplet size, the concentration gradient caused by selective evaporation leads to a density gradient, which can result in gravity-controlled flow.

In Chapter 3, we gradually increased the degree of complexity by adding a small amount of silicone oil into the binary system studied in Chapter 1 . We observed a Rayleigh-Taylor instability by segregation in such an evaporating microdroplet.

In Chapter 4, we studied a surfactant-laden glycerol-water evaporating system. The surfactant can mix with glycerol with the help of water. During the evaporation, the depletion of water leads to a segregation of surfactants through the formation of crystals.

In Chapter 5, we propose a robust method to suppress the "coffee-stain" effect by letting droplets evaporate on lubricated surfaces. It is demonstrated that by adding a certain amount of surfactants, thereby tuning the surface energy of droplets, we can manipulate the wetting state, flow behavior and final particle deposition. 
Finally, we conclude and summarize the work presented in this thesis. 



\section{Chapter 1}

\section{Evaporation-triggered segregation of sessile binary droplets*}

Droplet evaporation of multicomponent droplets is essential for various physiochemical applications, e.g. in inkjet printing, spray cooling and microfabrication. In this work, we observe and study phase segregation of an evaporating sessile binary droplet, consisting of a miscible mixture of water and a surfactant-like liquid (1,2-hexanediol). The phase segregation (i.e., demixing) leads to a reduced water evaporation rate of the droplet and eventually the evaporation process ceases due to shielding of the water by the non-volatile 1,2-hexanediol. Visualizations of the flow field by particle image velocimetry and numerical simulations reveal that the timescale of water evaporation at the droplet rim is faster than that of the Marangoni flow, which originates from the surface tension difference between water and 1,2-hexanediol, eventually leading to segregation.

*Published as: Yaxing Li, Pengyu Lv, Christian Diddens, Huanshu Tan, Herman Wijshoff, Michel Versluis, Detlef Lohse, Evaporation-triggered segregation of sessile binary droplets, Phys. Rev. Lett. 120, 224501 (2018). Experiments are done by Li and Lv. Numerical simulation is done by Diddens. 



\subsection{Introduction}

The evaporation of a sessile droplet has attracted a lot of attention over the past years $[4-6,9,12,15-24]$, not only from a fundamental scientific perspective, but also because of many technological and biological applications, such as inkjet printing [25], nanopatterning depositions [26], and DNA stretching [27]. Within the whole class of problems, the so-called "coffee-stain effect" which was presented to the scientific community 20 y ago [12], has become paradigmatic. The problem and its variations keep inspiring the community. This holds not only for the evaporation of liquids with dispersed particles $[21,28]$, but also for that of liquid mixtures, including binary and ternary mixtures [24,29-32]. In recent work on an evaporating Ouzo drop (a ternary mixture of water, ethanol and anise oil), Tan et al. [24] showed that a phase transition and the nucleation of oil microdroplets can be triggered by evaporation. The reason for the nucleation lies in the varying solubility of oil in the ethanol-water mixtures: the high evaporation rate at the rim of the droplet together with the higher volatility of ethanol as compared to water causes an oil oversaturation at the rim, leading to localized oil microdroplet nucleation. The oil microdroplets are advected over the whole drop by Marangoni flow and further droplets later nucleate in the bulk. Finally, the microdroplets are jammed and coalesce during the further evaporation process, eventually leading to the formation of a separated oil phase in the remaining binary water/oil droplet. Liquid-liquid phase separation during evaporation not only occurs for Ouzo drops, but is omnipresent in nature and technology [33-36].

In this work, we experimentally and numerically study segregation within an evaporating 1,2-hexanediol/water miscible binary droplet, which will turn out to follow a completely different route - even qualitatively - than that in an evaporating Ouzo drop, in which immiscible oil microdroplets nucleate. 1,2-hexanediol is used in a variety of applications, such as co-surfactant for modifying the sodium dodecyl sulfate (SDS) micelles [37] and oil solubilization in ternary systems [38]. The features of its aqueous solution are widely studied in many previous papers [39-41], which show that 1,2-hexanediol molecules form micelle-like aggregates characterized by a critical micelle concentration (CMC) in aqueous solutions, leading to an almost constant surface tension above the CMC [42]. Compared with water, 1,2-hexanediol is non-volatile under room conditions, implying a preferred evaporation of the more volatile water during the drying process. However, to the best of our knowledge, the segregation of the miscible 1,2-hexanediol and water during the evaporation 
process has never been observed, nor studied.

\subsection{Experimental results}

We begin with the visualization of the distribution of the mixture components during evaporation by labelling water and 1,2-hexanediol with the fluorescent dyes dextran and nile red, respectively. A dyed $0.5 \mu \mathrm{L}$ binary droplet with initial $10 \%$ mass concentration of 1,2-hexanediol (around the CMC [42]) is deposited on a transparent hydrophobic octadecyltrichlorosilane (OTS)-glass surface, while monitoring its evaporation under ambient conditions with confocal microscopy from side and bottom (see supporting information). The contact angle of the droplet varies between $43^{\circ}$ and $23^{\circ}$ during the whole evaporation process, measured by bright-field imaging from side view. Fig. 1.1 presents the segregation process of the evaporating binary droplet. In the beginning the droplet is homogeneously mixed, as revealed by the uniform green colour over the surface and on the bottom (Fig. 1.1A,1.1 $A^{\prime}$ ). About $34 \mathrm{~s}$ after deposition, 1,2-hexanediol microdroplets nucleate at the rim of the droplet, revealed by the yellow colour (Fig. $1.1 B, 1.1 B^{\prime}$ ). During further evaporation, the nucleated microdroplets of 1,2-hexanediol grow and coalesce, which forms starshape binary mixture area revealed in blue colour (Fig. $1.1 C, 1.1 C^{\prime}$ ). Eventually, 1,2-hexanediol covers the whole surface of the droplet and the evaporation process stops with some water being entrapped by the 1,2-hexanediol (Fig. $1.1 D, 1.1 D^{\prime}$ ). From comparing the initial and the final size, we calculate that approximately $96 \%$ of the water has evaporated while $4 \%$ got trapped.

To obtain insight into the segregation process, we record the evolution of the flow field within the evaporating binary 1,2-hexanediol/water droplet by particle image velocimetry (PIV) combined with confocal microscopy. For a first qualitative understanding, we added $1 \mu \mathrm{m}$ diameter fluorescent particles at a concentration of $5 \times 10^{-5} \mathrm{vol} \%$, which is much less than the particle concentration required for a quantitative PIV measurement [31,32]. The whole droplet and all particles were illuminated: particles near the substrate (pink colour) were in focus of the camera; the grey or transparent objects were out-of-focus particles and reside in the upper part of the droplet.

Initially, the flow is directed radially outwards near the substrate (see Fig 1.2A). In this phase, only water evaporates from the binary droplet and the droplet is thin, $H / L \ll 1$, where the droplet height $H$ is approximately $60 \mu \mathrm{m}$ and droplet footprint diameter $L$ is about $600 \mu \mathrm{m}$. Therefore, due to the relative high concentration of 1,2-hexanediol caused by the singularity of the water 

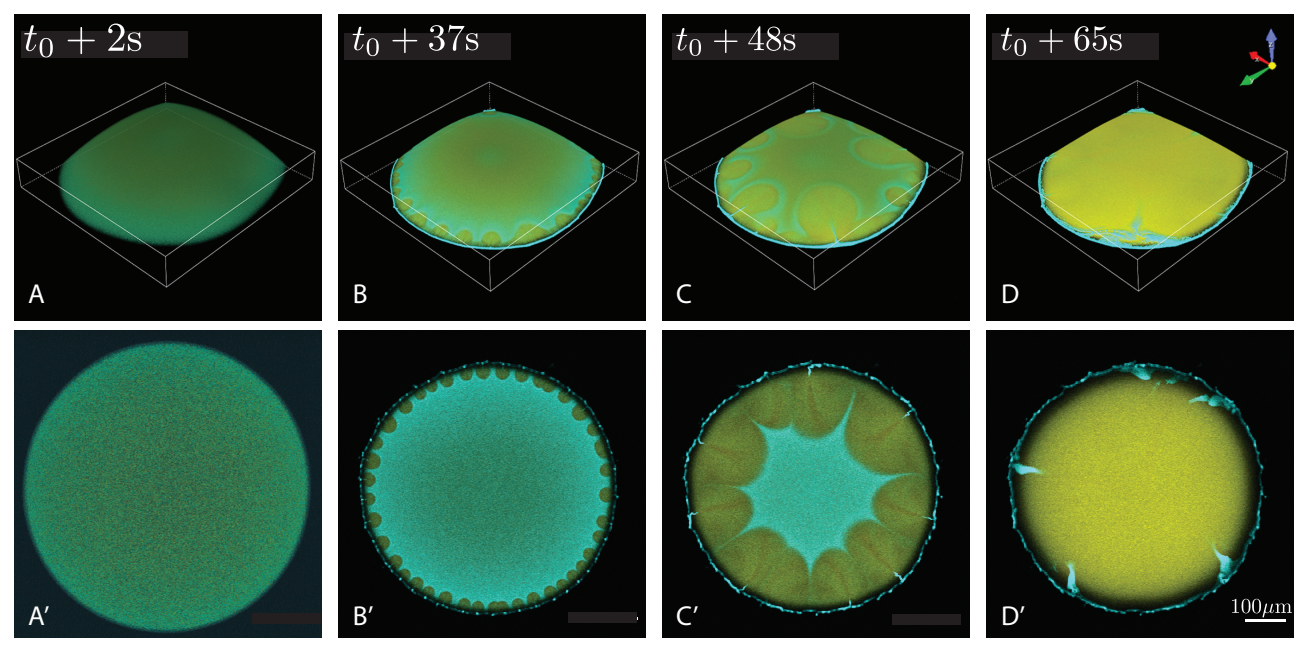

Figure 1.1: Confocal images of the segregation process during droplet evaporation in a semi-sideview (A-D) and bottom view (A'-D') taken at the same times. (A-D) The confocal microscope scans the rectangular box with the volume $590 \mu \mathrm{m} \times 590 \mu \mathrm{m} \times 90 \mu \mathrm{m}$. Water (blue) and 1,2-hexanediol (yellow) are labeled with different dyes for the observation. (A and A') In the beginning, the droplet is homogeneously mixed. (B and B') At about $34 \mathrm{~s}$ after recording started, 1,2-hexanediol nucleates at the contact line of the droplet, which is revealed as yellow round shapes. (C and C') The nucleated microdroplets of 1,2-hexanediol gradually grow and coalesce. (D and D') The evaporation ends when 1,2-hexanediol fully covers the surface of the droplet.

evaporation rate at the rim of the sessile droplet [16], a Marangoni flow is driven from the contact line to the apex of the droplet by the surface tension gradient, which originates from the concentration variation along the surface. Note that the surface tension of 1,2-hexanediol aqueous solution is monotonously decreasing with 1,2-hexandiol concentration when it is lower than the CMC [42]. The Marangoni flow, together with the evaporative water loss at the initially pinned rim, imply a replenishing convective flow inside the drop, radially outwards towards the rim. From fig. 1.3 we observe that the typical outwards flow velocity is $U \sim 10 \mathrm{\mu m} \mathrm{s}^{-1}$, implying a Reynolds number $R e=\rho H U / \mu \sim 10^{-4}$, where $\rho \approx 1 \times 10^{3} \mathrm{~kg} / \mathrm{m}^{3}$ is the liquid density and $\mu \approx 10 \mathrm{mPa}$ s is the viscosity.

We compare the corresponding time scale of the convective flow from the cen- 
ter towards the edge of the drop $t_{\mathrm{co}} \sim L / U$ with the time scale of evaporation $t_{\mathrm{ev}} \sim \rho L H /\left(D_{\mathrm{w}, \text { air }} \Delta c_{w}\right)$ [43], where $D_{\mathrm{w}, \text { air }}=2.4 \times 10^{-5} \mathrm{~m}^{2} / \mathrm{s}$ is the diffusion coefficient of water vapor at room temperature and $\Delta c_{\mathrm{w}} \approx 1 \times 10^{-2} \mathrm{~kg} / \mathrm{m}^{3}$ is the vapor concentration difference from the air-liquid interface to the surrounding air. We find that these two time scale are comparable, $t_{\mathrm{ev}} / t_{\mathrm{co}} \sim$ $\rho H U /\left(D_{\mathrm{w}, \text { air }} \Delta c_{\mathrm{w}}\right) \approx 1$. This indicates that the evaporative water loss is the main origin of the replenishing convective flux, and not the Marangoni flow from the rim of the drop towards its apex which otherwise would have led to better mixing. Consequently, the concentration of 1,2-hexanediol near the contact line keeps increasing.

In the second phase, after about $18 \mathrm{~s}$ (Fig. 1.2B), all particles, which had accumulated at the contact line, released and simultaneously moved upward along with the Marangoni flow $[31,44]$. We call this phenomenon de-staining, as it the opposite to what leads to the so-called coffee stain effect [12]. The particles move along the liquid-air interface due to their hydrophilicity and the diol accumulation at the rim. In the third regime (Fig. 1.2C), the particle-filled upper (water-rich) region is squeezed by growing nucleated microdroplets. A star-shaped pattern emerges. At the fingers of the star, the particles flow down to the bottom of the droplet. Compared with the observations in Fig.1.1, the star-shaped pattern corresponds to the blue part in Fig.1.1C and C', which represents the water-rich area. The fingers are formed by the liquid on the upper layer flowing downward through the streams between each two neighbouring growing nucleated microdroplets. During the segregation process, the surface tension force is dominant compared to gravity forces, as the Bond number $\mathrm{Bo}=\rho g L^{2} / \sigma \approx 10^{-1}$, where $g=9.8 \mathrm{~m} / \mathrm{s}^{2}$ gravity and $\sigma \approx 24 \mathrm{mN} / \mathrm{m}$ is the surface tension of the 1,2-hexanediol aqueous solution above the CMC [42]. In the final phase, when 1,2-hexanediol almost entirely covers the surface and the evaporation ceases, particles flow irregularly and eventually are deposited uniformly with no particles accumulating at the edge when evaporation fully stops (Fig. 1.2D).

To obtain a quantitative analysis of the flow field during evaporation, we add $520 \mathrm{~nm}$ diameter fluorescent particles at a concentration of $2 \times 10^{-3} \mathrm{vol} \%$ into the droplet. The flow speed $U$ and the wall-normal vorticity $\omega=\partial_{x} u_{y}-\partial_{y} u_{x}$ for the in-plane velocity $\left(u_{x}, u_{y}\right)$ are measured during the whole evaporation process. Also from the evolution of the mean vorticity $\bar{\omega}$, the different life phases of the evaporation can be identified, now even quantitatively, see Fig. 1.3. In the early phase, there is almost only outward radial flow, resulting in constant low vorticity. After de-staining of the particles, there are 


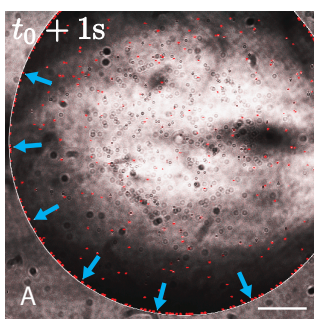

E. Before segregation: (A-B)

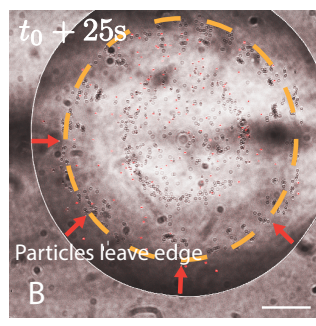

Higher surface

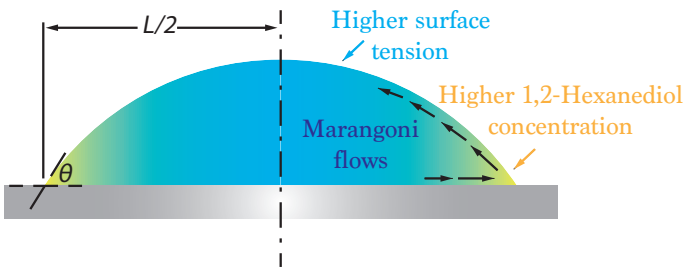

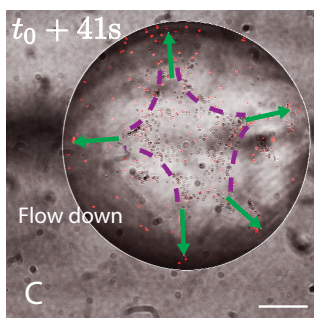

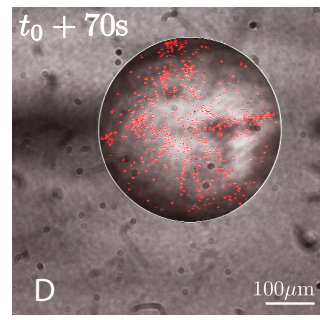

F. After segregation: (C-D)

Figure 1.2: (A-D) Bottom-view snapshots of the droplet seeded with fluorescent particles in different life phases. (A) The flow is directed radially outwards near the substrate, as shown by particles transported to the contact line (blue arrows). (B) All the particles are released from the contact line and flow to the upper center (orange circle) of the droplet (red arrows). (C) The particle-filled upper (water-rich) region is squeezed by growing nucleated microdroplets. A star-shaped pattern (purple dashed line) emerges. At the fingers of the star (green arrows), the particles flow down to the bottom of the droplet. (D) When the droplet stops evaporating, the particles are deposited homogeneously on the substrate, without leaving a coffee-stain. (E-F) Schematics of the flow inside the binary droplet at different phases. (E) Before segregation, the surface tension gradient drives a Marangoni flow from the edge to the apex of the droplet. (F) After segregation, the nucleated microdroplets of 1,2-hexanediol grow and coalesce. At the same time, water-rich liquid from the upper layer of the droplet flows down through the streams between neighbouring nucleated microdroplets.

some small vortices appearing near the droplet rim due to the receding contact line. When segregation starts, the vorticity sharply increases due to a series of vortices forming in the nucleated microdroplets of 1,2-hexanediol, see also in Fig. 1.1C. During coalescence of the growing nucleated microdroplets, small vortices merge and form larger vortices. When the growing microdroplets reach the area where floating particle reside, the particles flow down to the 
bottom. Finally the flow becomes irregular and then vanishes at the end of the evaporation process.

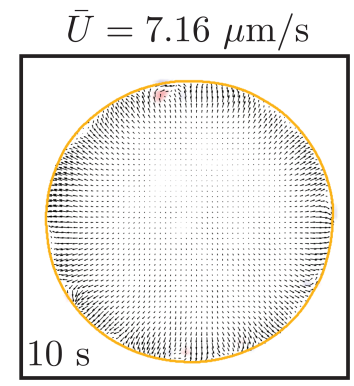

$\bar{U}=14.71 \mu \mathrm{m} / \mathrm{s}$

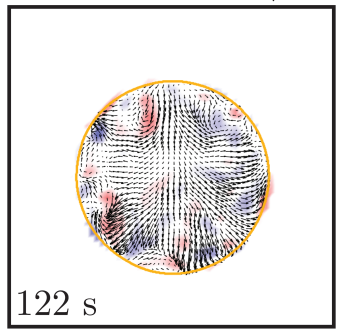

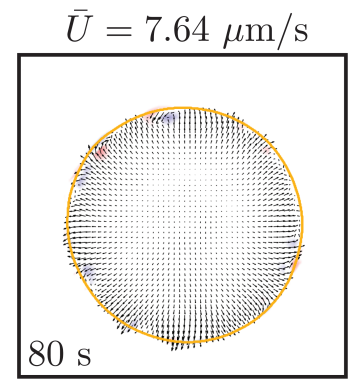

$\bar{U}=4.10 \mu \mathrm{m} / \mathrm{s}$

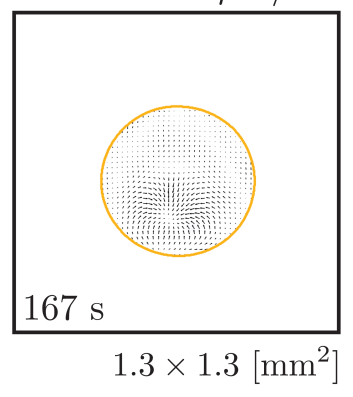

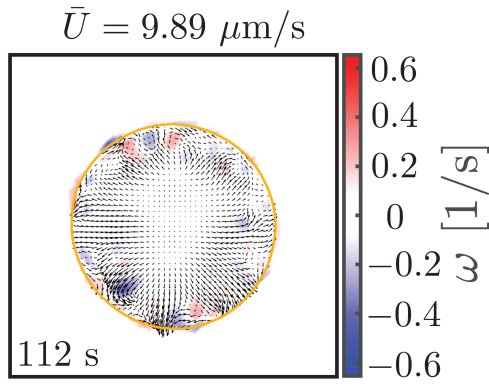

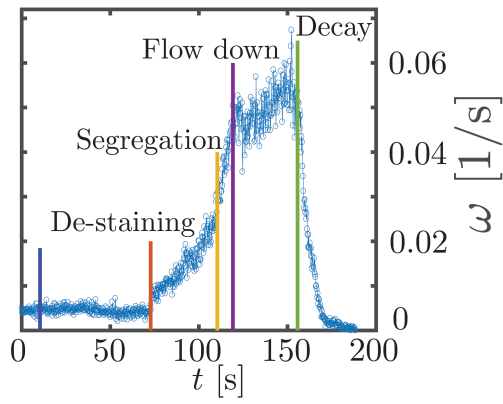

Figure 1.3: Particle image velocimetry results showing the flow field near the substrate in terms of velocity vectors and vorticity, allowing to identify evaporation stages. The five vertical lines show the moments of the five snapshots. (Blue line: 10s; red line: 80s; yellow line: 112s; purple line: 122s; green line: 167s.)

Sessile droplet evaporation is a diffusion-dominated process driven by the concentration gradient of the droplet's constituent(s) from the droplet interface towards the surroundings. The case of a pure evaporating sessile droplet has analytically been solved by Popov [5], see the supporting information.

\subsection{Numerical simulations}

For a droplet consisting of more than one component, the situation gets more complicated and can only be treated numerically. Several generalizations are necessary to adopt Popov's model to a multi-component droplet. Since these generalizations are described in detail in several recent publications $[24,32,45-$ 
47], only a brief overview of the model is given in the following, focusing on the case of the present binary mixture.

As 1,2-hexanediol is non-volatile, only the evaporation rate of water has to be determined. However, in contrast to the case of a pure water droplet, where the water vapor concentration $c_{\mathrm{w}}$ is saturated directly above the liquid-gas interface, i.e. $c_{\mathrm{w}}=c_{\mathrm{w}, \mathrm{s}}$, in the case of a droplet, consisting of two miscible liquids, the vapor concentration is given by the vapor-liquid equilibrium. This equilibrium can be expressed by Raoult's law, i.e. $c_{\mathrm{w}}=\gamma_{\mathrm{w}} X_{\mathrm{w}} c_{\mathrm{w}, \mathrm{s}}$, where $X_{\mathrm{w}}$ is the mole fraction of water in the liquid and $\gamma_{\mathrm{w}}$ is the activity coefficient of water for the 1,2-hexanediol/water mixture. The water vapor concentration is in general non-uniform along the interface and changes over time. The evaporation process is modeled by the quasi-steady vapor diffusion equation $\nabla^{2} c_{\mathrm{w}}=0$. We use Raoult's law at the liquid-gas interface and the ambient vapor concentration $c_{\mathrm{w}}=c_{\mathrm{w}, \infty}$ far away from the droplet as Dirichlet boundary conditions. The evaporation rate of water $J_{\mathrm{w}}$ is then given by the diffusive flux at the interface, i.e. $J_{\mathrm{w}}=-D_{\mathrm{w}, \text { air }} \partial_{n} c_{\mathrm{w}}$.

In case of a pure droplet, or for a multicomponent droplet in the presence of a very intense Marangoni flow, it is sufficient to keep track of the total mass of each species over time to predict the volume evolution [45,47]. Here, however, the Marangoni flow is weak and segregation occurs, so that an explicit spatiotemporal dependence of the local liquid composition emerges. Hence, the convection-diffusion equation for the water mass fraction $Y_{\mathrm{w}}$ has to be solved inside the droplet:

$$
\rho\left(\partial_{t} Y_{\mathrm{w}}+\vec{u} \cdot \nabla Y_{\mathrm{w}}\right)=\nabla \cdot\left(\rho D \nabla Y_{\mathrm{w}}\right)-J_{\mathrm{w}} \delta_{\text {interf. }}
$$

The mass density of the liquid $\rho$ and the diffusivity $D$ are compositiondependent quantities, i.e. $\rho\left(Y_{\mathrm{w}}\right)$ and $D\left(Y_{\mathrm{w}}\right)$. The evaporation rate of water enters Eq. (1.1) as interfacial sink term $\delta_{\text {interf. }}$.

The advection velocity $\vec{u}$ is obtained from the Stokes equation, subject to a no-slip boundary condition at the substrate, the kinematic boundary condition considering evaporation, the Laplace pressure in normal direction at the liquid-gas interface, and the Marangoni shear stress that arises due to the composition-dependent surface tension $\sigma\left(Y_{\mathrm{w}}\right)$ in tangential direction at the liquid-gas interface. Furthermore, the composition-dependence of the dynamic viscosity $\mu\left(Y_{\mathrm{w}}\right)$ has to be considered. For the composition-dependence of the liquid's material properties, we have fitted experimental data and/or used models. More details and plots of these relations can be found in the supplementary information. 
The resulting set of coupled equations can be solved numerically with a finite element method $[32,46,47]$. We restrict ourselves to axial symmetry. Since the evolution of the contact angle is determined by microscopic interactions at the contact line, it cannot be predicted by the model. Instead, the experimentally measured evolution of the contact angle was imposed throughout the simulation, see Fig. 1.4A.
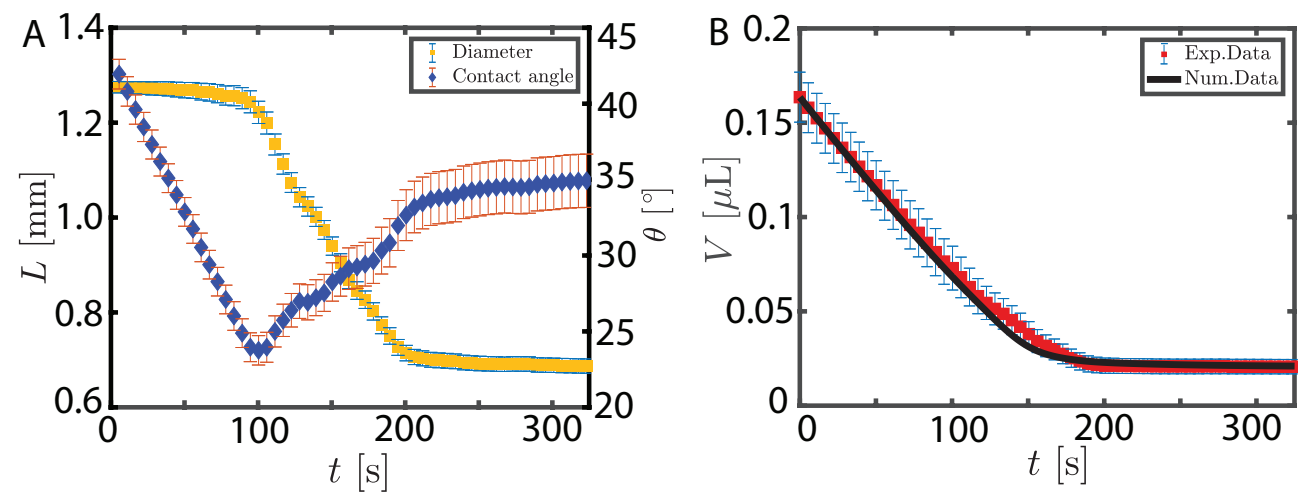

Figure 1.4: Experimental (data points) and numerical results (solid line) for the temporal evolution of the geometrical parameters (A): footprint dimater $L$, contact angle $\theta$ and (B): volume $V$ from experiment and numerical simulation. The error bars are deduced from the experimental accuracy.

In Fig. 1.5, these snapshots of the simulation for the droplet consisting of an initial 10\% 1,2-hexanediol are depicted. While initially a considerable Marangoni flow is present and the profile of the evaporation rate resembles the case of a pure water droplet, the situation drastically changes at later times: The Marangoni flow ceases due to the nearly constant surface tension at lower water concentrations. Towards the end of the evaporation process, the evaporation rate suddenly decreases once the water concentration $Y_{\mathrm{w}}$ falls below a threshold of about $10 \%$. Since this transition sets in near the contact line, the profile of the evaporation rate shows a remarkable deviation from the case of a pure droplet with a pronounced evaporation at the apex in this stage. The evaporation-triggered segregation effect in radial direction is well captured by the model. Finally, a remaining water residue is entrapped in the bulk of the droplet ( $4 \%$ of the initial water content) which can only reach the interface by diffusion. The comparison between simulation and experimental data shows an excellent quantitative agreement as shown in Fig. 1.4B. 


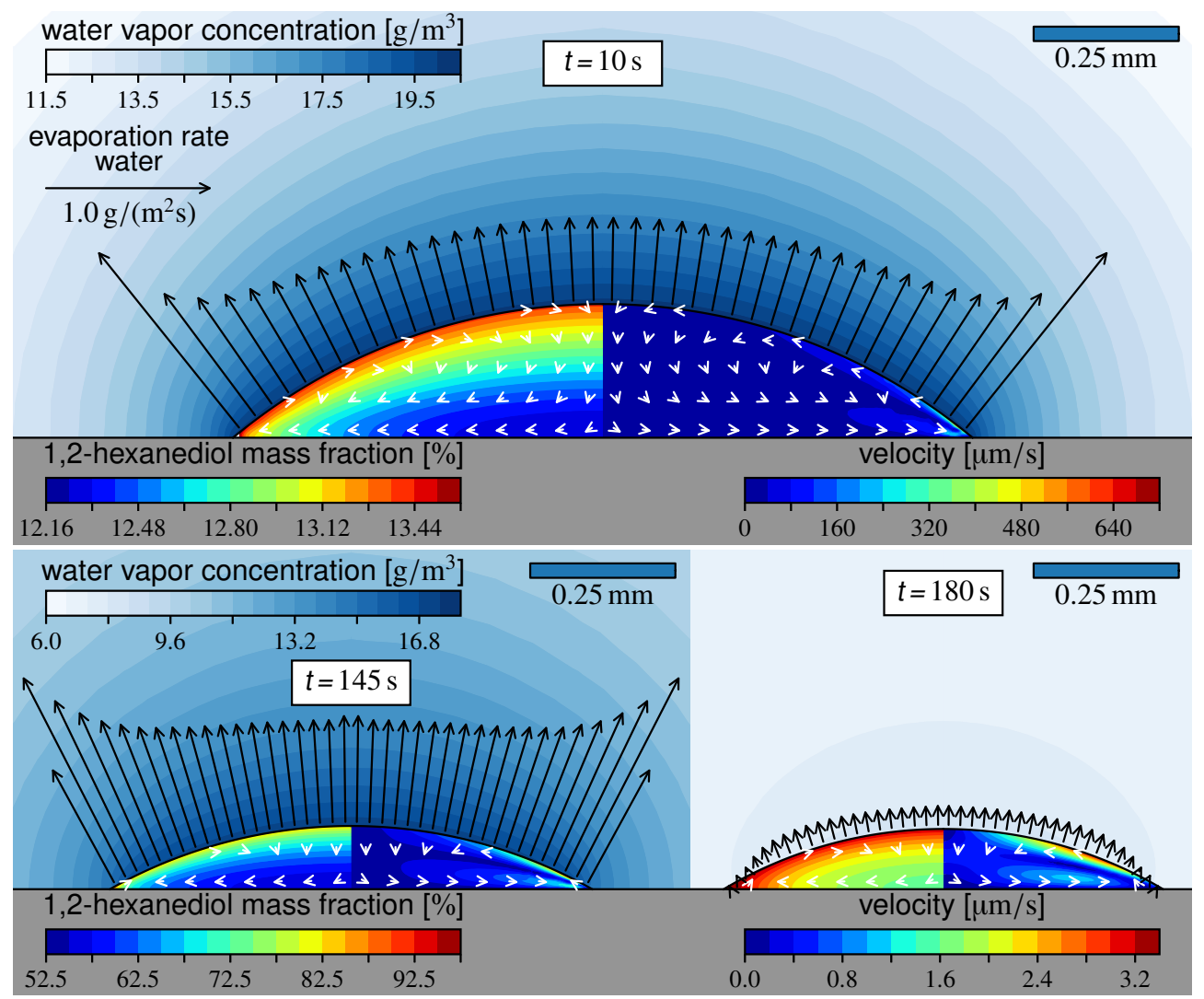

Figure 1.5: These snapshots from the simulation of an evaporating droplet with initially $10 \%$ of 1,2-hexanediol with the axisymmetric finite element model at different times $t$. In the gas phase, the water vapor concentration $c_{\mathrm{w}}$ is shown and the corresponding evaporation rate $J_{\mathrm{w}}$ is indicated by the arrows at the interface. Inside the droplet, the mass fraction of 1,2-hexanediol (left) and the velocity (right) is depicted. Note the very different phenomena at $t=10 \mathrm{~s}$ (upper) and at the two later times $t=145 \mathrm{~s}$, and $t=180 \mathrm{~s}$ (lower).

\subsection{Conclusions and discussions}

To summarize, segregation within a binary droplet in spite of the simplifying asymmetry, triggered by selective evaporation is observed during the drying process of a 1,2-hexanediol/water mixture droplet. The small surface tension differences cannot drive a strong enough Marangoni flow on the surface to 
induce a high enough convection within the droplet to obtain perfect mixing. Therefore a locally high concentration of 1,2-hexanediol accumulates near the contact line of the droplet, leading to segregation. This is the first time to reveal this mechanism of segregation, which is very surprising and counterintuitive, given that 1,2-hexanediol and water are fully miscible. The evolution of the vorticity field indicates different life stages of the evaporating droplet. We quantitatively compare the experimental data with numerical simulations of the convective-diffusion equations, showing excellent agreement. While the model perfectly predicts the water and diol concentrations in the inner center and outer layer of the droplet, respectively, note that it cannot predict the phase separation of the two liquids due to the complexity of the diol's solubility in water. Indeed, 1,2-hexanediol can mix with water at any concentration without phase separation in equilibrium due to the formation of micelles-like aggregates. However, in the dynamic system of an evaporating droplet, the continuous loss of water leads to large fluctuations through mutual attractions of micelles within the new 1,2-hexanediol phase, which eventually forms the nucleation of 1,2-hexanediol [48]. From an energetic point of view, it is likely that the separated 1,2-hexanediol phase has the same, or at least a very similar, chemical potential as the mixed phase in the droplet [36]. Stochastic fluctuations then lead to the phase separation. Our findings offer new perspectives to understand how surfactants act in an evaporating system, and may inspire further studies of complex dynamical aspects associated with microdroplet nucleation.

Finally, we note that very recently, in a parallel but independent study on drying of a binary droplet, Kim \& Stone [49] also find that the evaporation of one component in the binary system can be so fast that it can lead to segregation, overcoming convective and diffusive mixing effects.

\subsection{Supplemental Materials}

\subsubsection{Experimental details}

The droplet mixture is obtained by mixing 1,2-hexanediol with purity of $\geqslant 98 \%$ (Sigma-Aldrich) and Milli-Q water. The transparent glass substrate the droplet was deposited on is hydrophobic, being coated with an OTS monolayer. The advancing and receding contact angle of water on this substrate is $110^{\circ}$ and $95^{\circ}$ respectively. The contact angle of 1,2 -hexanediol used in the experiments on this substrate is $40^{\circ} \pm 3^{\circ}$ measured with a video-based optical 
contact angle measurement system (DataPhysics OCA15 Pro), see Fig. 2.6b. Before being used, the substrates were cleaned by 15 -min sonication in $99.8 \%$ ethanol and 5 min in Milli-Q water sequentially and subsequently dried with compressed $\mathrm{N}_{2}$ flow for 30 sec. In the experiments, droplets evaporate under stable laboratory conditions, and the relative humidity $R H$ and the ambient temperature $T$ are monitored and maintained constant in each measurement, which are around $30 \%$ and $22{ }^{\circ} \mathrm{C}$, respectively.

Confocal microscopy was employed to visualize the concentration distribution of water and 1,2-hexanediol within the binary droplet, see Fig. 2.6(a). Realtime observations were carried out by using an inverted Nikon A1 confocal laser scanning microscope system (Nikon Corporation, Tokyo, Japan) with a $20 \times$ dry objective (CFI Plan Apochromat VC $20 \times / 0.75$ DIC, numerical aperture $=0.75$, working distance $=1.0 \mathrm{~mm}$ ). The binary droplet was labeled with two different dyes, i.e., Nile Red and Dextran. Nile Red is a lipophilic dye which dissolves only in 1,2-hexanediol and was excited by a laser at a wave length of $561 \mathrm{~nm}$, while Dextran preferentially dissolves in water and was excited by a laser at a wave length of $488 \mathrm{~nm}$ simultaneously. Two types of images were acquired to reveal the dynamical process of the segregation during evaporation. First, two-dimensional (2D) images were captured by focusing at the bottom of the droplet, i.e., near the liquid-solid interface. Secondly, three-dimensional (3D) images were obtained by reconstruction from a series of consecutive Z-stack images scanned from the substrate to the top of the droplet. The scan started as soon as the droplet was deposited on the glass substrate. Operating in resonant mode, the scan rate for 2D images was 30 fps, while each Z-stack scan loop for 3D images took approximately $4 \mathrm{~s}$ to complete. This timescale is much smaller than that of evaporation so that the variations of the droplet during the scans can be neglected. Both $2 \mathrm{D}$ and $3 \mathrm{D}$ time-series images were integrated and compiled as animations to show the details of the evaporating process.

To analyse the evaporation behaviour quantitatively, the droplet was imaged during the entire evaporation lifetime and photographed at constant time intervals. The set-up (see Fig. 2.6(b)) was utilised to take the side-view images which allows us to measure volume, contact angle, footprint diameter and volume loss at each time step.

For flow visualization, we performed Particle Image Velocimetry (PIV) by adding fluorescent particles [microParticles GmbH; PS-FluoRed-5.0: Ex/Em $530 \mathrm{~nm} / 607 \mathrm{~nm}$ ] into the working fluids. The PIV measurement is also implemented on confocal microscopy setup, see Fig. 2.6(c). The particles were 
excited by a laser at wave length of $561 \mathrm{~nm}$ and the fluorescent signals were captured at a frame rate of 25 fps. We begin with a visualisation of flow field within the whole droplet to get a qualitative 3D information of the flow field within the whole droplet. We added $1 \mu \mathrm{m}$ diameter fluorescent particles at concentration of $5 \times 10^{-5} \mathrm{vol} \%$. The whole droplet and the particles are illuminated: particles near the substrate which reveal pink colour are focused by camera; ones which reveal grey or transparent colour are de-focused due to locating on upper layers of the droplet. By this method, we observe the flow field within the whole droplet. The limitation is that except the particle in the bottom layer near substrate, we cannot distinguish the precise vertical positions of the particles in de-focused planes. Then we measure the flow field near the substrate quantitatively by adding $520 \mathrm{~nm}$ diameter fluorescent particles at a concentration of $2 \times 10^{-3}$ vol\% into the droplet. The in-plane flow speed $U$ and the wall-normal vorticity $\omega=\left[\left(\partial u_{y} / \partial x\right)-\left(\partial u_{x} / \partial y\right)\right]$ for in-plane velocity $\left(u_{x}, u_{y}\right)$ are measured through the whole evaporation process [31,32].

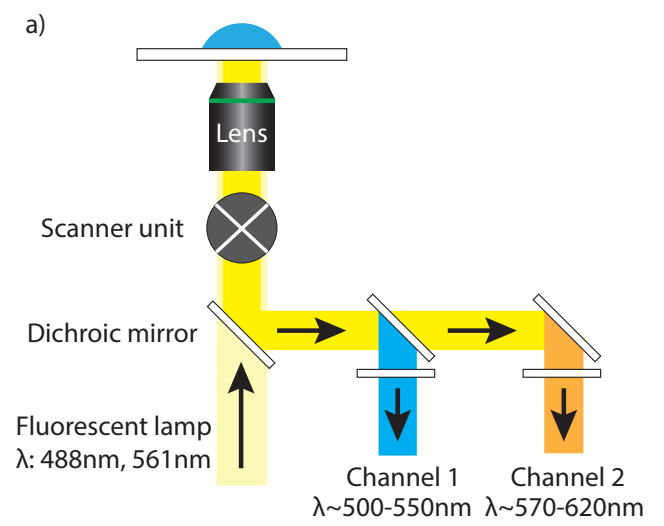

b)

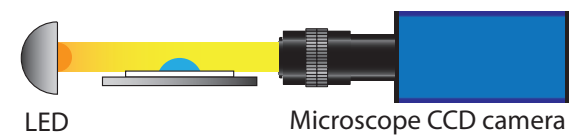

c)

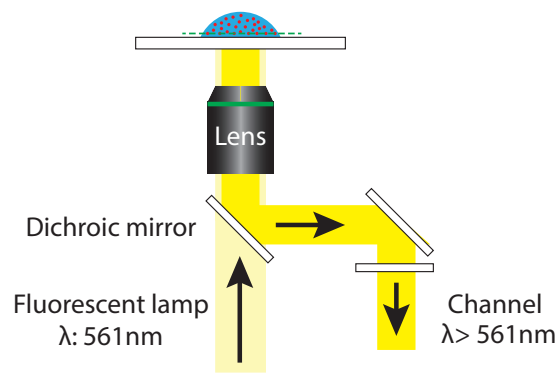

Figure 1.6: Experimental apparatus. (a) Schematic of the confocal microscopy for the components distribution visualization. (b) The setup to image the evaporation of droplets by the side view. (c) Particle Image Velocimetry setup.

\subsubsection{Image analysis and post processing}

All the image analyses and post processing were done with Matlab R2016b. For the volumetric measurement, the image analysis was implemented using a custom-made Matlab code to detect the droplet profile with sub-pixel accuracy [50]. Then we use a spherical cap to fit the profile and obtain the contact 
angle, footprint radius and the volume of the droplet. The error bar is deduced from the inaccuracy of determining the exact profile of the droplet, which is three blur pixels. For the PIV image, we first remove the background outside the droplets and export a new series of images for the image processing. Then we use a Matlab application "PIVlab" $[51,52]$ to analyze the images and obtain the wall-normal vorticity $\omega=\partial u_{\mathrm{y}} / \partial x-\partial u_{\mathrm{x}} / \partial y$ for the in-plane velocity $\left(u_{\mathrm{x}}, u_{\mathrm{y}}\right)$.

\subsubsection{Diffusion model for evaporation of sessile droplets}

From a theoretical perspective, the evaporation of a droplet is a diffusiondominated process driven by the concentration gradient of the droplet's constituent(s) from the droplet interface towards the surrounding medium. The case of a pure sessile droplet evaporating in air has been solved by Popov [5] in 2005:

$$
\frac{\mathrm{d} M}{\mathrm{~d} t}=-\frac{\pi}{2} D_{w} L\left(c_{\mathrm{s}}-c_{\infty}\right) f(\theta)
$$

with

$$
f(\theta)=\frac{\sin (\theta)}{1+\cos (\theta)}+4 \int_{0}^{\infty} \frac{1+\cosh (2 \theta \varepsilon)}{\sinh (2 \pi \varepsilon)} \tanh [(\pi-\theta) \varepsilon] \mathrm{d} \varepsilon
$$

where $L$ and $\theta$ are the base diameter and contact angle of the droplet, respectively. Three parameters determine the diffusion process: the diffusion constant $D_{\mathrm{w}}$ of the droplet constituent in air; the concentration $c_{\mathrm{s}}$ at the dropletair interface, which always equals to the saturated concentration at given temperature and pressure; and the concentration $c_{\infty}$ far from the droplet, which is determined by the relative humidity $R H, c_{\infty}=R H c_{\mathrm{s}}$.

\subsubsection{Composition-dependent liquid properties}

For an accurate simulation, the composition-dependence of the liquid properties is of fundamental importance. To that end, experimental data for the surface tension $\gamma$ [42] and the viscosity $\mu$ [53] has been fitted. Due to the unavailability of experimental data for the mass density and the diffusivity, assumptions have been made. The mass density $\rho$ is assumed to be given by ideal mixing, i.e. by the harmonic average of the pure densities weighted by their mass fractions. For the diffusivity $D$, first the diffusivity $D_{Y_{\mathrm{w}} \rightarrow 1}=1.38 \times 10^{-9} \mathrm{~m}^{2} / \mathrm{s}$ of infinitely dilute 1,2-hexanediol in water has been estimated by the method 
of Hayduk and Minhas [54]. The composition-dependence is then assumed to follow a Stokes-Einstein law, i.e. $D=(D \mu)_{Y_{\mathrm{w}} \rightarrow 1} / \mu$. The activity coefficient $\gamma_{\mathrm{w}}$ has been calculated by the thermodynamic model AIOMFAC [55,56], which is based on the experimental data of Marcolli et al. [57]. Plots of all relations are depicted in Figure 1.7.
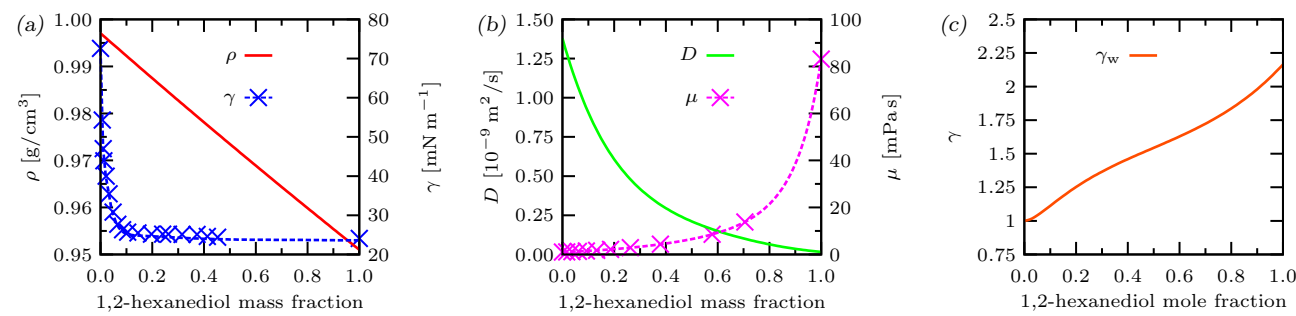

Figure 1.7: Composition-dependence of the liquid properties. Where available, the data was obtained by fitting experimental data $[42,53]$ (indicated by points). Otherwise, models were used. 


\section{Chapter 2}

\section{Gravitational effect in evaporating binary microdroplets*}

The flow in an evaporating glycerol-water binary sub-millimeter droplet with Bond number Bo $\ll 1$ is studied both experimentally and numerically. First, we measure the flow fields near the substrate by micro-PIV for both sessile and pendant droplets during the evaporation process, which surprisingly show opposite radial flow directions - inward and outward, respectively. This observation clearly reveals that in spite of the small droplet size, gravitational effects play a crucial role in controlling the flow fields in the evaporating droplets. We theoretically analyze that this gravity-driven effect is triggered by the lower volatility of glycerol which leads to a preferential evaporation of water then the local concentration difference of the two components leads to a density gradient that drives the convective flow. We show that the Archimedes number Ar is the nondimensional control parameter for the occurrence of the gravitational effects. We confirm our hypothesis by experimentally comparing two evaporating microdroplet systems, namely a glycerol-water droplet and a 1,2-propanediol-water droplet. We obtain different Ar, larger or smaller than a unit by varying a series of droplet heights, which corresponds to cases with or without gravitational effects, respectively. Finally, we simulate the process numerically, finding good agreement with the experimental results and again confirming our interpretation.

*Published as: Yaxing Li, Christian Diddens, Pengyu Lv, Herman Wijshoff, Michel Versluis and Detlef Lohse, Gravitational effect in evaporating binary microdroplets, Phys. Rev. Lett. 122, 114501 (2019). Experiments are done by Li and Lv. Numerical simulation is done by Diddens. 



\subsection{Introduction}

The evaporation of a microdroplet on a flat substrate has attracted a lot of attention because of its beautiful and phenomenologically rich fluid dynamics $[4-6,9,12,15-18,20,21,23,24]$ and its relevance in various technological applications, such as medical diagnostics [22] and the fabrication of electronic devices [19]. For many of these applications, an understanding of the internal flow structure is crucial. One example is the so-called "coffee stain problem" [12], i.e. an evaporating colloidal drop in which an outward capillary flow along the substrate carries the dispersed material from the interior towards the pinned contact line. This seminal study opened up a new line of research for surface coatings and patterning technologies, which is crucial for various applications in inkjet printing [25], 3D printing technology [58] and molecular biology [27].

However, in nearly all of these applications, the droplet liquid is not pure, but a binary or even ternary liquid. As is well known, then Marangoni flow, which is driven by surface tension gradients, is coming into play [16,59-62], strongly affecting the evaporative behavior. The variation of the surface tension originates from two mechanisms or the combination of both, namely a temperature gradient $[59,60]$ or a solute concentration gradient [24,49,61-64], due to the spatially varying local evaporation rates at the droplet surface. The conventional understanding is that the flows within sub-millimeter droplets can only be attributed to capillary and Marangoni convections, while natural convection is considered to be negligible as the surface tension force is dominant compared to gravity forces [60]. The Bond number of such a small-sized droplet system reads $\mathrm{Bo}=\rho g R^{2} / \gamma \ll 1$, which normally implies a gravity-independent system [31]. However, this only holds as a measure of the importance of the gravity force compared to the surface tension while relating to the droplet shape [65]. In recent years, studies of evaporating aqueous $\mathrm{NaCl}$ droplets revealed that natural convection can be driven by evaporation-induced density gradients inside a colloidal droplet [66-70]. However, to our best knowledge, an internal flow controlled by gravitational effects has never been observed, nor experimentally confirmed in a drying microdroplet consisting of two miscible liquid components.

In this work, we investigate the flow field inside an evaporating glycerol-water binary miscible droplet. Glycerol is a very common liquid heavily used in industry [71] and laboratory experiments [72], in particular due to its strong hygroscopic nature [73]. Recently, Shin et al. [74] found a spontaneous Bénard- 
Marangoni (BM) convection within the water-glycerol system: the hygroscopic absorption of water vapor at the interface generates thermal and solutal gradients, leading to a surface tension gradient, thus sustaining a BM instability. They confirmed that buoyancy is not the driving force by observing the same convection cells when orienting the system upside-down. Here, however, we study the reverse process to absorption: the evaporation of a dilute aqueous glycerol droplet. In this system, glycerol can be considered nearly non-volatile under room temperature as compared to water, implying a selective evaporation of the more volatile water, which leads to a concentration gradient of the components in the drying droplet. Such a concentration gradient generates surface tension and density gradients, which drives a convective flow in the droplet. Remarkably, the flow field is mainly controlled by the buoyancy force through the density gradient.

\subsection{MicroParticle Image Velocimetry}

We implemented microparticle image velocimetry $(\mu \mathrm{PIV})$ to measure the flow fields within the binary droplets with opposite configuration: a sessile and a pendant droplet. We deposited $(0.18 \pm 0.03) \mu \mathrm{L}$ binary droplets (glycerol:water, $50: 50 \%$ by weight), seeded with fluorescent microspheres $(520 \mathrm{~nm}$ diameter) at a concentration of $2 \times 10^{-2} \mathrm{vol} \%$ on a OTS glass substrate. Glycerol has a higher density $\rho_{\mathrm{g}}=1.261 \times 10^{3} \mathrm{~kg} / \mathrm{m}^{3}$ and lower surface tension $\gamma_{\mathrm{g}}=64 \times 10^{-3} \mathrm{~N} \mathrm{~m}^{-1}$ than water $\left(\rho_{\mathrm{w}}=0.998 \times 10^{3} \mathrm{~kg} / \mathrm{m}^{3}, \gamma_{\mathrm{w}}=\right.$ $\left.75 \times 10^{-3} \mathrm{~N} / \mathrm{m}\right)$. During evaporation, the ambient temperature and relative humidity were stable, i.e., $T=21 \pm 1{ }^{\circ} \mathrm{C}$ and $\mathrm{RH}=50 \pm 5 \%$. During the drying process the contact angles $\theta$ for both the sessile and the pendant droplet were between $90^{\circ}$ to $104^{\circ}$. A typical droplet initially has a footprint radius $R_{0}=400 \mu \mathrm{m}$ and a height $h_{0}=490 \mu \mathrm{m}$. We investigate the flow fields for both sessile and pendant droplets by adjusting the optical focus plane at different heights within the droplets: at $10 \mu \mathrm{m}$ (i) and at $200 \mu \mathrm{m}$ (ii) away from the substrate, as labeled in Fig 2.1. For sessile droplets, we observe an inward radial flow in the middle of the droplet (a1) and an outward radial flow close to the base of droplet (a2). Fig 2.1.(a3) presents the schematics of the flow velocity within the axis plane of the droplet from side view: the black arrows represent the measured flow velocities and the red arrows indicate the flow pattern reconstructed from the measurement. In contrast, Fig 2.1.(b1,b2) show a completely reversed flow fields for pendant droplets. The flow velocity near the substrate (b2) is radially outward, from center towards the contact 
line, which is opposite to the one of sessile droplet (b2). In the middle of the droplet (b1), it shows relatively weaker inward radial flow only in the outer region and asymmetric annulus flow near the edge of the droplet. Fig 2.1.(b3) illustrates the flow pattern in a pendant droplet.

(a1)

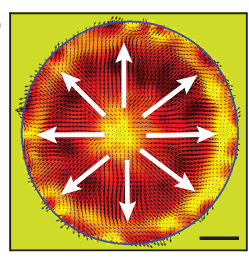

(a3)

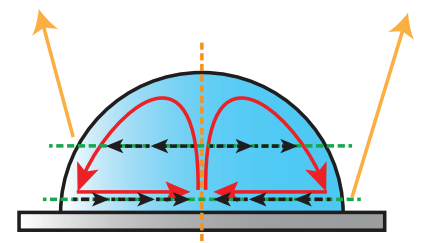

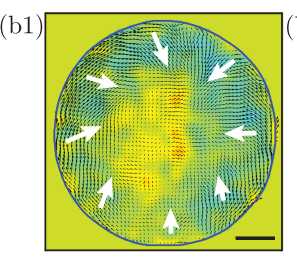

(b3)
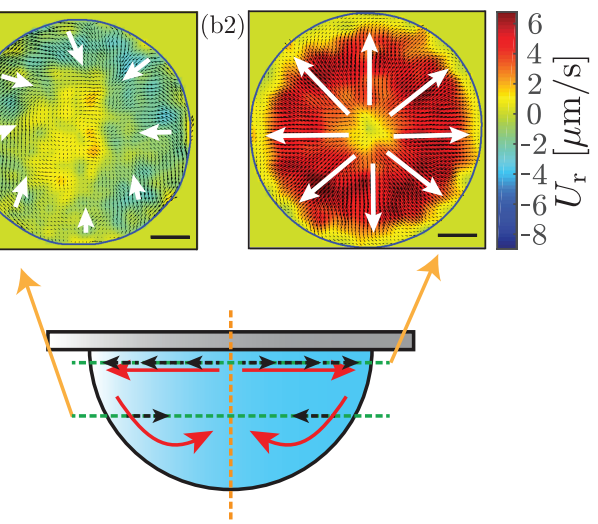

Figure 2.1: The micro-PIV measurement of flow fields in both sessile (a) and pendant (b) droplets. The scale bars represent $200 \mu \mathrm{m}$. (a1 and a2) Flow fields in a sessile binary droplet measured at different heights: $200 \mu \mathrm{m}$ and $10 \mu \mathrm{m}$ away from the substrate, respectively. The white arrows represent the flow direction. (a2) The measurement near the substrate shows an inward radial flow, (a2) the one at larger height reveals an outward radial flow. (b1 and b2) Flow fields of a pendant binary droplet measured by the same method as the sessile droplets. (b2) The flow near the substrate follows outward radial direction, (b1) but the flow at midheight reveals an annular flow with deviations from axisymmetry near the edge and irregular flow within the inner part. The four PIV images were taken with four different droplets. (a3 and b3) The schematics of the flow pattern in side views of both sessile and pendant droplets.

The opposite radial flow directions near the substrate of differently orientated droplets clearly indicates that the gravitational effect is dominating to control the flow structure. In our system, the gravitational effect is driven by the density gradient in the bulk of the droplet, which in turn is generated from concentration gradients induced by the selective evaporation of the more volatile water. As the density of the glycerol-water mixture monotonously increases with increasing glycerol concentration, the local density decreases from the outer layer towards the inner bulk. For the sessile droplet, the denser 
glycerol-rich part is collecting on the top of the droplet, resulting in an unstable situation: denser glycerol-rich part suspends atop lighter water-rich part. As sketched in Fig 2.1.(a3), the lighter liquid part rises up due to the buoyancy, pushing the denser liquid to sink along the outer layer, hence an inward flow is passively driven from the contact line towards the center in the bottom layer. In contrast, for pendant droplets, the glycerol-rich part is at the bottom of the liquid bulk which cannot drive a buoyancy flow in the center. Instead, the denser liquid near the contact line flows down along the outer layer, and is replenished by the outward radial flow close to the substrate, implying a much weaker gravitational effect in the pendant droplet. Moreover, due to the intense coupling between composition and flow, instabilities can arise which lead to axial symmetry breaking near the interface [46,61].

We can estimate the flow velocity in the evaporating binary droplet by scaling arguments. The typical velocity from the $\mu \mathrm{PIV}$ results is $U \approx 10 \mu \mathrm{m} \mathrm{s}^{-1}$ so that the Reynolds number $\operatorname{Re}=\rho U R / \mu_{\mathrm{m}} \approx 10^{-4}$, where $\mu_{\mathrm{m}} \approx 5 \mathrm{mPas}$ is the viscosity of the binary mixture. Furthermore, the Péclet number $\mathrm{Pe}=$ $U R / D \approx 10$, with the mutual diffusion coefficient $D \approx 0.4 \times 10^{-9} \mathrm{~m}^{2} / \mathrm{s}$, implying advection is dominating over diffusion. Assuming a quasi-steady flow, the gravitational force due to the density difference is balanced by the viscous shear stress, which scales as $g \Delta \rho_{\mathrm{m}} \sim \mu \nabla^{2} U$. The density $\rho_{\mathrm{m}}$ of the mixture liquid varies with the relative concentration $\phi$ following the Boussinesq approximation, $\rho_{\mathrm{m}}=\rho_{0}\left[1+\beta\left(\phi-\phi_{0}\right)-\alpha\left(T-T_{0}\right)\right]$ [75], where $\rho_{0}, \phi_{0}$ and $T_{0}$ denote, the density, concentration ratio and temperature of the reference state, respectively, and where $\alpha$ and $\beta$ indicate the thermal and solutal expansion coefficient, respectively. Note that the evaporation of water cools down the liquid temperature near the surface, which enhances the density difference between the surface area and the bulk. For now we neglected the thermal effect and only considered the density changes due to concentration differences. This assumption is validated by numerical simulations (see below) with and without the thermal contribution with only minor changes to the flow field. Thus the density difference is given by $\Delta \rho_{\mathrm{m}}=\rho_{0} \beta \Delta \phi$, with the solutal expansion coefficient is $\beta \approx 0.2$.

The evaporation rate is controlled by diffusion of water vapor molecules to the surrounding air. Therefore, the mass loss $\Delta m$ within a typical timescale $\Delta t \sim$ $R / U$ can be estimated from $\Delta m \sim D_{\mathrm{w}, \text { air }} \Delta C_{\mathrm{w} \text {,air }} R \Delta t$, with $D_{\mathrm{w} \text {,air }} \approx 10^{-5} \mathrm{~m}^{2} / \mathrm{s}$ is the diffusion coefficient of water vapor at room temperature and $\Delta C_{\mathrm{w}, \text { air }} \approx$ $10^{-2} \mathrm{~kg} / \mathrm{m}^{3}$ is the water vapor concentration difference between the air-liquid interface and the surrounding air. Hence, the concentration ratio difference 
$\Delta \phi$ can be calculated from $\Delta \phi \sim \Delta m / m_{0} \sim\left(D_{\mathrm{w}, \text { air }} \Delta C_{\mathrm{w}, \text { air }} R \Delta t\right) /\left(2 \pi R^{3} \rho_{0} / 3\right)$. Therefore, we obtain the characteristic steady-state flow velocity $U_{\mathrm{c}}$ as

$$
U_{\mathrm{c}} \sim\left(\frac{3 D_{\mathrm{w}, \text { air }} \Delta C_{\mathrm{w}, \mathrm{air}} R}{2 \pi \mu_{\mathrm{m}}} g \beta\right)^{1 / 2} \sim 10^{-5} \mathrm{~m} \mathrm{~s}^{-1},
$$

thus in very good agreement with the velocity estimate from experiment. Note that Eq. 2.1 is independent of the ratio of nonvolatile glycerol to water. We test the applicability of the scaling approach on various initial mass concentration of glycerol, ranging from $10 \%$ to $60 \%$. The measured temporal evolutions of the averaged radial velocity near the substrate for sessile droplets are shown in Fig 2.2(a). We then rescale the experimental data by the characteristic velocity $U_{\mathrm{c}}$ and time scale $\tau_{\mathrm{c}}$. The latter is estimated by the diffusion lifetime of a sessile droplet [4],

$$
\tau_{\mathrm{c}}=\frac{\rho_{\mathrm{m}} R^{2}}{D_{\mathrm{w}, \text { air }} \Delta C_{\mathrm{w}, \text { air }}} .
$$

As shown in Fig 2.2(b), with the rescaling all data collapse, except the early stages of the droplets with $10 \%$ and $20 \%$ initial concentration. We conclude that the gravitational effect is enhanced by increasing the concentration of glycerol, before reaching the concentration at which glycerol starts to absorb the humidity from the air.

\subsection{Theoretical analysis}

We now introduce the Archimedes number $\mathrm{Ar}=g h^{3} \rho_{0} \Delta \rho / \mu_{\mathrm{m}}^{2}[76]$ as the control parameter for this problem, where $h$ is the height of droplets. For large Ar $\gg 1$ gravity plays a prominent role, whereas for small $\mathrm{Ar} \ll 1$, the gravity can be neglected. To test the applicability of Ar, we varied the height of a series of sessile glycerol-water droplets, with $h_{\mathrm{g}}=608 \mu \mathrm{m}, 514 \mu \mathrm{m}, 320 \mu \mathrm{m}$ and $154 \mu \mathrm{m}$. For our system, with a density difference between glycerol and water $\Delta \rho_{\mathrm{g}}=263 \mathrm{~kg} / \mathrm{m}^{3}$, we obtain $\mathrm{Ar}=23.1,14.0,3.4$ and 0.37 . A prominent inward radial flow in the center of the droplets is observed for the cases when Ar is greater than 1, which indicates gravity-dominating flow. However, for the case when $\mathrm{Ar}<1$, this flow disappears. For comparison, we investigated the occurrence of gravitational effects in a 1,2-propanediol-water binary droplet in the same experimental setup. The density for 1,2-propanediol is $1.036 \times 10^{3} \mathrm{~kg} / \mathrm{m}^{3}$ at room temperature, which is slightly greater than that of water (density difference $\Delta \rho_{\mathrm{p}}=36 \mathrm{~kg} / \mathrm{m}^{3}$ ) and $\mu_{\mathrm{p}} \approx 7 \mathrm{mPa}$ sor the viscosity [77]. As with glycerol, 1,2-propanediol is also nearly non-volatile at room 

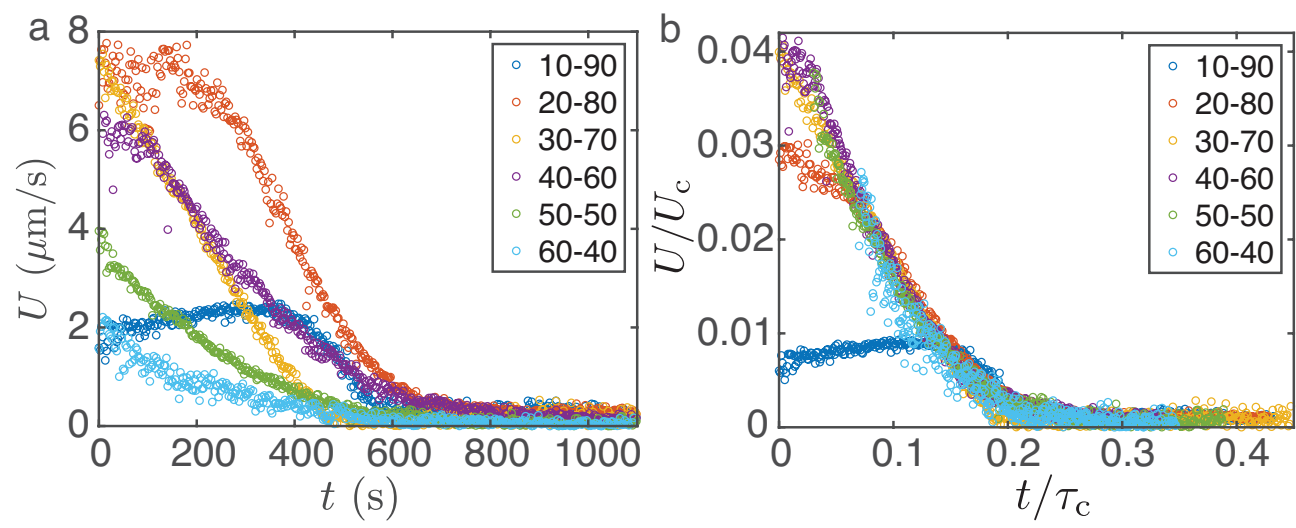

Figure 2.2: (a) The evolutions of the averaged radial velocity near the substrate for sessile droplets with various initial glycerol mass concentrations, $10 \%$ (blue), $20 \%$ (red), 30\% (orange), $40 \%$ (purple), $50 \%$ (green) and $60 \%$ (cyan). (b) The dimensionless averaged radial velocity plotted against the dimensionless time for the same data as in (a), which are scaled by the characteristic velocity and life time according to Eq. 2.1 and Eq. 2.2, respectively. All the scaled data collapse into each other except the early stages of the ones with $10 \%$ and $20 \%$ initial concentration.

temperature conditions, resulting in a preferential evaporation of water. The contact angles $\theta_{\mathrm{p}}$ for sessile and pendant droplets are between $70^{\circ}$ and $75^{\circ}$ due to the low surface tension of 1,2-propanediol [78], indicating a stronger Marangoni effect than that of the glycerol-water droplets. We measured the flow field in an optical focal plane $10 \mu \mathrm{m}$ above the substrate for oppositely configured droplets (sessile and pendant) with two different droplet heights, $h_{\mathrm{p}} \approx 800 \mu \mathrm{m}$ and $410 \mu \mathrm{m}$, with $\mathrm{Ar} \approx 3.7$ and 0.5 , respectively, as shown in Fig. 2.3. We observed the opposite radial outward flows in large droplets with different orientations, similar to glycerol-water droplets. But for small droplets, we find the same radially outwards flow direction for both droplets: there is no detecable flow within the center of the droplet, but only outward radial flow in the outer regime, which clearly indicates that for these droplets with low Ar, only the Marangoni effect dominates the flow near the edge. The two liquid systems clearly show that the Archimedes number is indeed the crucial control parameter for the occurrence of the gravitational effects. 
Without gravitational effect

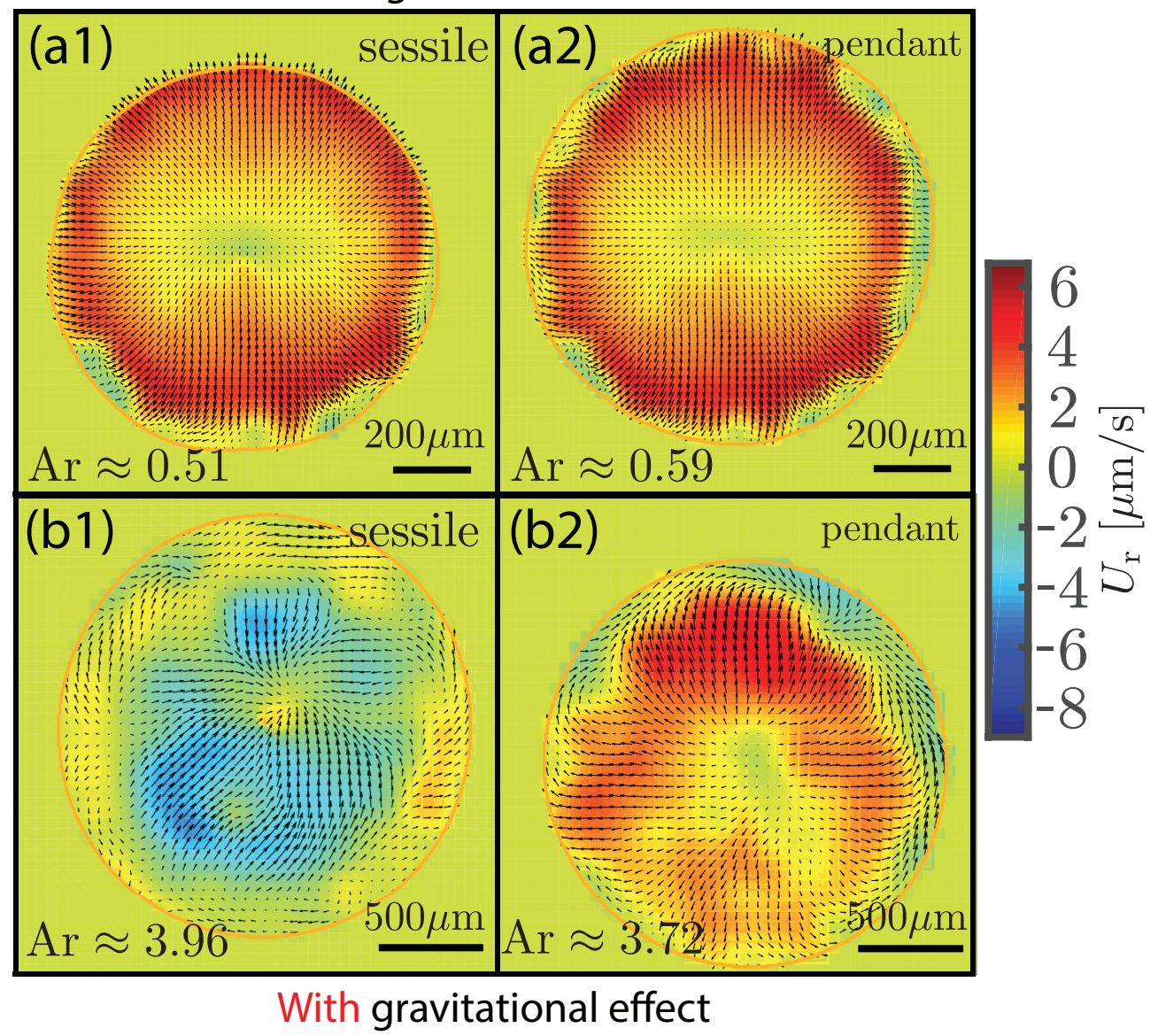

Figure 2.3: Micro-PIV measurement of the flow field for both sessile and pendant 1,2-propanediol-water droplets with different heights: $h_{g}=410 \mu \mathrm{m}$ (a1), $425 \mu \mathrm{m}$ (a2), $820 \mu \mathrm{m}$ (b1) and $800 \mu \mathrm{m}$ (b2). The flow fields were measured at same height: $10 \mu \mathrm{m}$ away from the substrate. (a1) and (a2) The small droplets have low $\mathrm{Ar}<1$. The flows within two different oriented droplets show same radial direction, which indicates no gravitational effect. (b1) and (b2) The large droplets have $\mathrm{Ar}>1$. The different radial flow directions clearly show gravitational effect. 


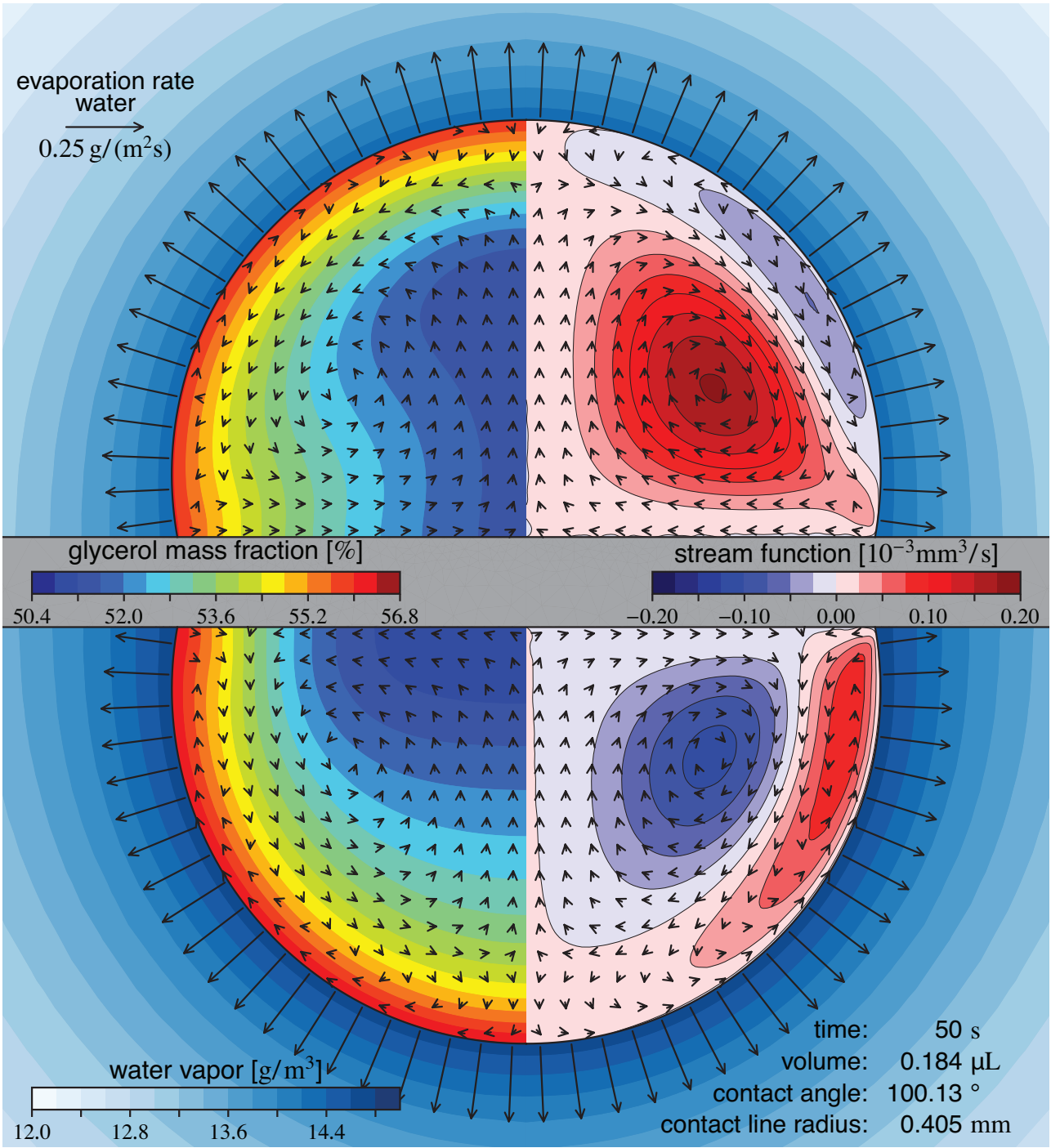

Figure 2.4: Snapshot of the numerical results for sessile (upper) and pendant (lower) droplets, respectively. In the gas phase, the water vapor concentration (color-coded) is shown along with the evaporation rate (arrows). Inside the droplet, the glycerol mass fraction (left) and the Stokes stream function (right) are depicted, where the arrows indicate the local flow direction.

\subsection{Numerical simulations}

To validate the experimental results, corresponding finite element simulations were performed. To that end, an axisymmetric model [46] was employed, which 
had already been successfully validated with binary water-ethanol and ternary ouzo droplets [32]. The model solves the diffusion equation for the water vapor concentration $C_{\mathrm{w} \text {,air }}$ in the gas phase, assuming vapor-liquid equilibrium according to Raoult's law. It includes the activity coefficient [57] of the two components at the liquid-gas interface and considers the ambient vapor concentration far away from the droplet. The diffusive water vapor flux at the interface determines the volume evolution and the normal component of the velocity in the droplet and is furthermore used as interfacial sink term in the convection-diffusion equation for the local composition in the droplet. The liquid properties, including the surface tension $\gamma$, mass density $\rho$, viscosity $\mu$, diffusivity $D$ and thermodynamic activity of water $a_{\mathrm{w}}$, are not constant, but coupled to the composition field. The composition-dependent properties of the glycerol-water mixture have been extracted from experimental data and are plotted in Refs. $[45,46]$. While the gravitational body force has been neglected in the original model [46], the discussed experimental results clearly indicate the relevance of this term. Hence, the generalized model used here solves the Navier-Stokes flow inside the droplet with the composition-dependent body force $\rho \vec{g}$ in the bulk and the Marangoni shear stress at the liquid-gas interface.

Figure 2.4 shows snapshots of the simulations for the sessile and the pendant droplet using the experimental parameters. It is apparent that, as in the microPIV results, close to the substrate the flow is directed inward/outward for the sessile/pendant droplet, whereas it is reversed in the bulk layer approximately at the center of the droplet. Along the liquid-gas interface the simulations reveal a counter-rotating vortex as is apparent from the Stokes stream function depicted in the right half of Fig. 2.4. For the pendant droplet, the flow at the interface is in the same direction as predicted by pure Marangoni flow in the absence of natural convection, i.e. from the apex towards the contact line. For the sessile droplet, however, the strong natural convection in the bulk transports water from the bulk to the apex. Thereby, despite of the enhanced evaporation rate at the top, there is a higher water concentration at the apex as compared to the contact line. This results in a Marangoni flow from the contact line towards the apex, i.e. in the opposite direction as predicted when considering the Marangoni effect without natural convection. Hence, the impact of the gravity is not only able to decisively control the flow direction in the bulk, but can also reverse the interfacial composition gradient and the corresponding Marangoni flow.

A quantitative comparison of the experimental and numerical results is shown in Fig 2.5. Here, the temporal evolution of the angular-averaged radial velocity 

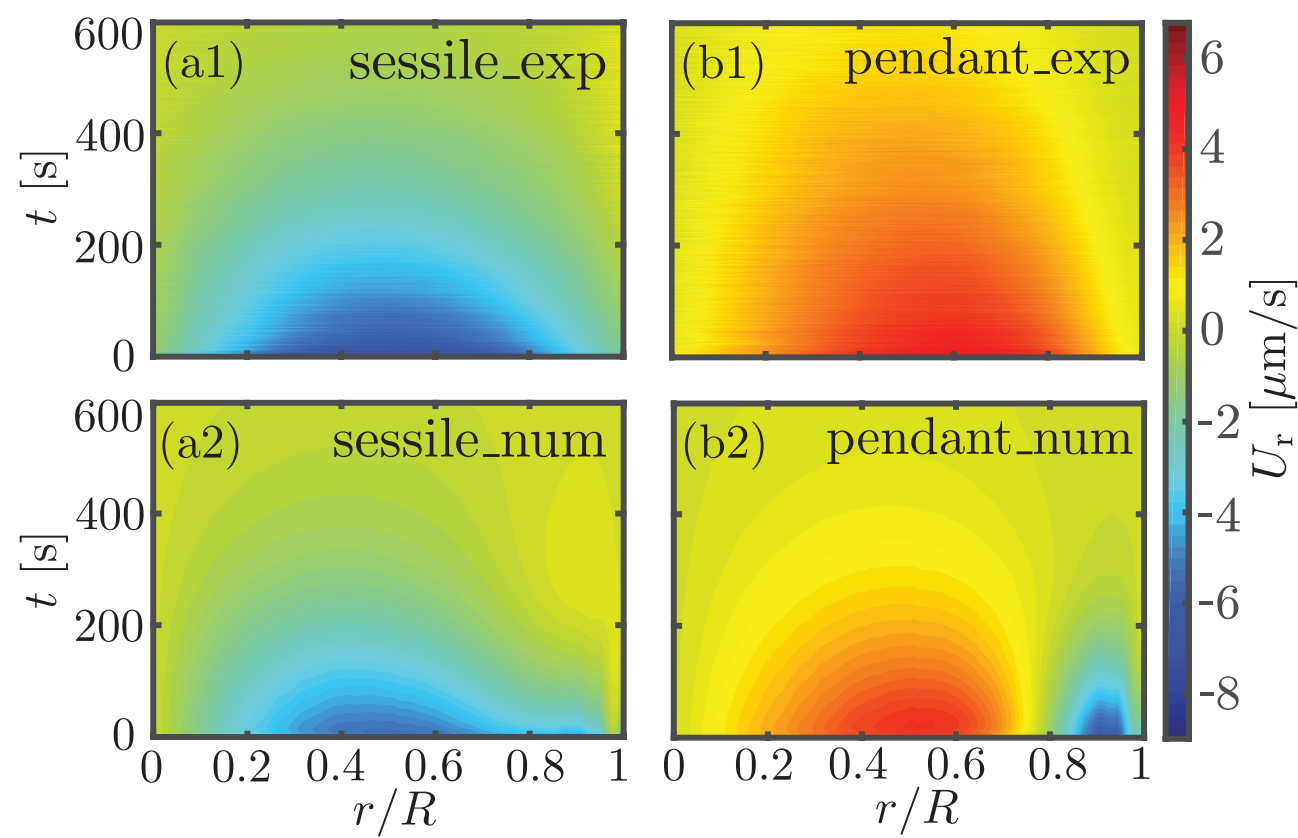

Figure 2.5: Experimental (a1, b1) and numerical (a2, b2) results for the evolution of the radial velocity near the substrate for both sessile (a1, a2) and pendant (b1, b2) droplets. Positive values indicates outward flow. For sessile droplets (a1, a2), the numerical simulation shows a great agreement with experiment. However, for pendant droplet (b1, b2), the numerical simulation shows a stronger flow near the contact line than in the experiment, which is presumably due to the axial symmetry breaking of the flow in experiment.

of the micro-PIV measurement in the plane close to the substrate is compared with the numerically obtained radial velocity evaluated at the same height. It is apparent that the radial velocity profile is in good agreement for the entire drying time. For the case of the pendant droplet, however, the simulation predicts an inward flow region close to the contact line, which was not found in the corresponding micro-PIV measurement. This difference can presumably be attributed to the assumption of axisymmetry in the model, a condition that may easily be broken for the pendant droplet system (cf. Fig 2.1(b1)). 


\subsection{Conclusions}

Gravitational effects triggered by density gradients due to selective evaporation can play a dominating role in controlling the flow in evaporating multicomponent droplets, even at the sub-millimeter scale and small Bond numbers. Misled by the small Bo, hitherto, most studies on this subject until now have disregarded the influence of natural convection. Our results show conclusively that natural convection can readily dominate the flow for $\mu \mathrm{L}$ droplets. Thus, our findings stimulate a careful treatment of the interplay of natural convection and Marangoni flow in multicomponent droplets in future studies. Furthermore, the possibility to reverse the bulk flow by overturning the system opens new application perspectives for surface coating and particle patterning. - On resubmission of our paper, we got aware of a reference which came to similar conclusion, however, employing different methods and different liquids [79].

\subsection{Supplemental Materials}

\subsubsection{MicroParticle Image Velocimetry}

For flow visualization, we performed MicroParticle Image Velocimetry ( $\mu \mathrm{PIV})$ by adding fluorescent particles [Fluoro-Max; Red Flourescent Polymer Microspheres: Ex/Em $530 \mathrm{~nm} / 607 \mathrm{~nm}$; Diameter: $0.52 \mu \mathrm{m}$ ] into the working fluids at concentration of $2 \times 10^{-2}$ vol\%. The $\mu \mathrm{PIV}$ measurement is implemented on a confocal microscope (Nikon Corporation, Tokyo, Japan) with a $10 \times$ dry objective (Nikon, Plan Fluor $10 \times / 0.30$, OFN25, DIC, L/N1), see Fig. 2.6. The particles were excited by a laser at a wave length of $561 \mathrm{~nm}$ and the fluorescent signals were captured at a frame rate of $25 \mathrm{fps}$. For the sessile droplet, the droplet is illuminated from the bottom and the fluorescent signal is also captured by the lens from the bottom. For the pendant droplet, by adding an objective inverter (InverterScope, LSM TECH), the droplet is illuminated and imaged from the top.

\subsubsection{Material preparations}

The droplet mixture is obtained by mixing glycerol (>99\%, Sigma-Aldrich) and Milli-Q water with 0.5:0.5 mass concentration. The transparent glass substrate we used is hydrophobic, being coated with an OTS monolayer [80]. The advancing and receding contact angles of water on this substrate are 


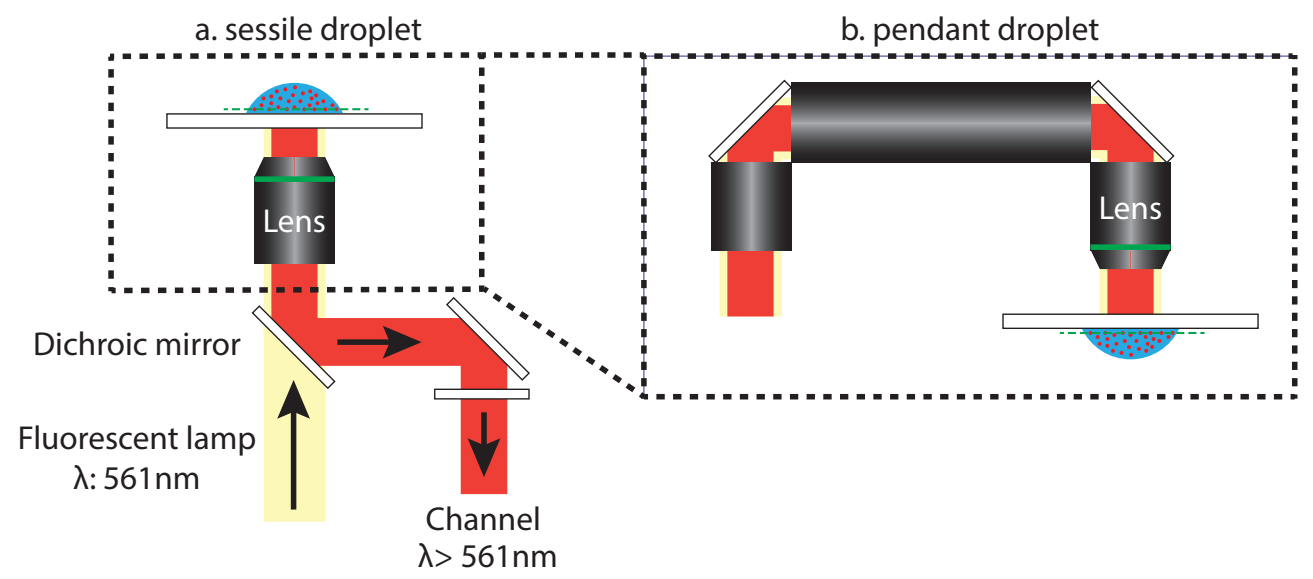

Figure 2.6: Schematic of the confocal microscope for MicroParticle Image Velocimetry. (a) The sessile droplet is illuminated from the bottom and the fluorescent signal is captured from the bottom lens. (b) The pendant droplet is illuminated and imaged from the top by adding an objective inverter.

measured with a video-based optical contact angle measurement system (DataPhysics OCA15 Pro). The droplets were deposited on the substrate by a Hamilton $2 \mu \mathrm{l}$ syringe, which was vertically mounted in a computer motorized pump, adding or withdrawing liquid into droplet with constant rate of $0.05 \mathrm{\mu l} / \mathrm{s}$. When the contact line was moving with constant contact angle, we measured the advancing or receding angle, which are $110^{\circ} \pm 3^{\circ}$ and $95^{\circ} \pm 3^{\circ}$, respectively.

The contact angle of the glycerol-water mixture droplet is $101^{\circ} \pm 3^{\circ}$ measured with the same image system. Before being used, the substrates were cleaned by 15 -min sonication in $99.8 \%$ ethanol and 5 min in Milli-Q water sequentially and subsequently dried with compressed N2 for $30 \mathrm{sec}$. In the experiments, the droplets evaporate under stable laboratory conditions, and the relative humidity $\mathrm{RH}$ and the ambient temperature $\mathrm{T}$ are monitored and maintained constant in each measurement, which are $50 \pm 5 \%$ and $21 \pm 1^{\circ} \mathrm{C}$, respectively.

\subsubsection{Experimental measurements for glycerol-water binary sys- tems with different droplet sizes}

We tested the applicability of Archimedes number with various droplet sizes in glycerol-water liquid system. As shown in Fig. 2.7, we obtained different 
Ar from 23.1 down to 0.37 in glycerol-water systems, which shows the gravitydriven flow weakens with decreasing Ar. When $\mathrm{Ar}<1$, the inward radial flow becomes almost invisible: the weak gravitational effect is almost completely damped by the viscosity in this scenario. The observations of the liquid system clearly show that Ar is the control parameter to predict the gravity effect in such systems.

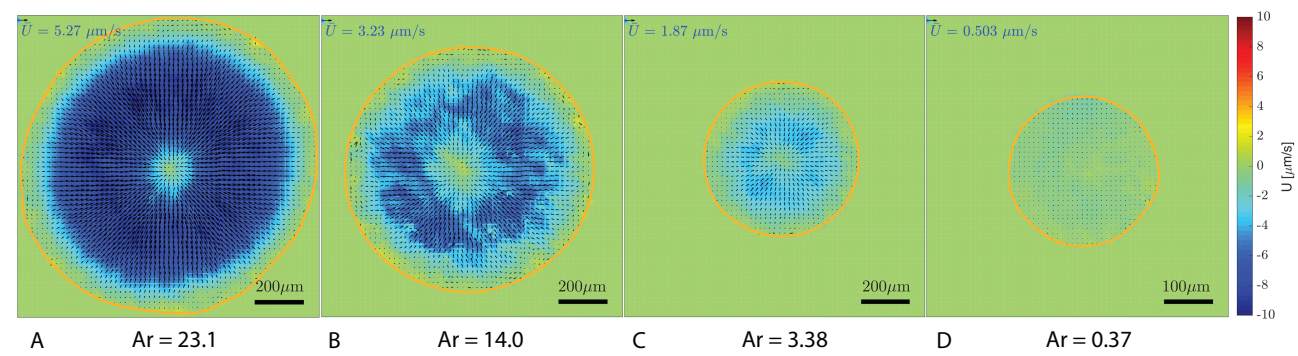

Figure 2.7: Micro-PIV measurements for glycerol-water sessile droplets with various heights: $h_{\mathrm{g}}=608 \mu \mathrm{m}, 514 \mu \mathrm{m}, 320 \mu \mathrm{m}$ and $154 \mu \mathrm{m}$.

\subsubsection{Numerical Simulations}

The finite element method that is used for the simulations is described in detail in Ref. [46]. The only difference is the consideration of the buoyancy term $\pm \rho g \vec{e}_{z}$, which has not been taken into account in the previous studies $[32,45,46]$. The incorporation of this additional body force term in the numerics is trivial. While our findings clearly reveal that the buoyancy force is fundamental for reproducing the experimentally observed flow in waterglycerol droplets, the flow in binary water-ethanol and ternary ouzo droplets is already well reproduced without the consideration of gravitational forces [32]. Although there is a large density difference between water and ethanol, the very intense and chaotic Marangoni flow prevents the emergence of sufficiently large density gradients in the bulk of these droplets, so that natural convection does not have a noticeable influence for the water-ethanol droplets. To substantiate this, we have repeated the simulation of the ethanol-water of Ref. [32] with consideration of the buoyancy term $\pm \rho g \vec{e}_{z}$. The statistics of the chaotic flow in the droplet remained nearly the same as compared to the simulations without consideration of gravity.

Due to the limitations of experiments, we performed the numerical simulations for both sessile and pendant glycerol-water droplets, with initial contact angle 
$\theta=60^{\circ}$, see them in supplementary movie 11 . From the movie we clearly see that with enhanced Marangoni effect, the gravitational effect still plays a dominating role in controlling the bulk flow close to the center. For the sessile droplet, the Marangoni flow near the surface has an opposite direction to the gravity-driven flow, but it is not sufficient to reverse the gravity-driven flow inside the droplet. 


\section{Chapter 3}

\section{Rayleigh-Taylor instability by segregation in an evaporating multicomponent microdroplet*}

The evaporation of multi-component droplets is relevant to various applications but challenging to study due to the complex physicochemical dynamics. Recently, [64] reported evaporation-triggered segregation in 1,2-hexanediol-water binary droplets. In this present work, we added $0.5 \mathrm{wt} \%$ silicone oil into the 1,2-hexanediol-water binary solution. This minute silicone oil concentration dramatically modifies the evaporation process as it triggers an early extraction of the 1,2-hexanediol from the mixture. Surprisingly, we observe that the segregation of 1,2-hexanediol forms plumes, rising up from the rim of the sessile droplet towards the apex during the droplet evaporation. By orientating the droplet upside down, i.e., by studying a pendant droplet, the absence of the plumes indicates that the flow structure is induced by buoyancy, which drives a Rayleigh-Taylor instability (i.e., driven by density differences $\&$ gravitational acceleration). From $\mu$ PIV measurement, we further prove that the segregation of the non-volatile component (1,2-hexanediol) hinders the evaporation near the contact line, which leads to a suppression of the Marangoni flow in this region. Hence, on long time scales, gravitational effects play the dominant role in the flow structure, rather than

*Based on: Yaxing Li, Christian Diddens, Tim Segers, Herman Wijshoff, Michel Verluis and Detlef Lohse, Rayleigh-Taylor instability by segregation in an evaporating multicomponent microdroplet, J. Fluid Mech. (2020) (In press). 
Marangoni flows. We compare the measurement of the evaporation rate with the diffusion model of [5], coupled with Raoult's law and the activity coefficient. This comparison indeed confirms that the silicone-oil-triggered segregation of the non-volatile 1,2-hexanediol significantly delays the evaporation. With an extended diffusion model, in which the influence of the segregation has been implemented, the evaporation can be well described. 



\subsection{Introduction}

Evaporation of sessile droplets has attracted a lot of attention over the past decades due to its ubiquitousness and huge relevance for various applications, such as inkjet printing [81], surface patterning [82], microfabrication [58], among others. In particular, the pioneering work of [12], unveiling the mystery of the so-called coffee-stain effect, has inspired many scientific studies on evaporating droplets over the past twenty years.

While the evaporation of single-component droplets is relatively well understood $[4,5,9,15,16,18,21,83]$, multi-component droplets show far more complex dynamics during the drying process. This is due to the complicated coupling of the mutual interactions between species $[11,32,84]$ and the resulting flow structures $[31,85,86]$. Essentially, the selective evaporation of each component is the reason underlying the complexity: the preferred evaporation of one component as compared to the other(s) can result in inhomogeneous liquid distributions. [61] first reported the sequential flow transitions in an evaporating ethanol-water binary droplet, which showed an evaporation-induced Marangoni instability in the early life stage. Many following studies [32,62,87] show that the solutal Marangoni stress driven by the surface tension gradient dominates the flow structure in evaporating multicomponent microdroplets. Very recently, [79] and [88] also found gravity-driven flows in different binary microdroplet systems, which are triggered by the density gradients from the selective evaporation. This is the first evidence that buoyancy-driven Rayleigh convection can overcome Marangoni flow in controlling the flow structure in such evaporating liquid-mixture droplets with Bond number Bo $\ll 1$.

For a specific category of multicomponent systems with a metastable phase regime, the phenomena are even more intriguing and complex. Tan et al [24, $47,89]$ systematically studied a ternary "Ouzo" system, which involves not only complex flow behaviours, but remarkably, multiple phase transitions, i.e., oil microdroplet nucleation and phase separation. Additionally, in a dissolution system, the interaction between host liquid and droplet liquids can also lead to segregation of the components inside the droplet [90,91]. Recently, Li et al [64] reported an unexpected segregation triggered by selective evaporation within a miscible 1,2-hexanediol-water binary droplet, in which 1,2-hexanediol is almost non-volatile compared to water. The insufficient replenishment of water from the droplet interior towards the contact line by the weak convection inside the droplet causes the local accumulation of 1,2-hexanediol in the contact line region, which eventually leads to segregation $[49,92]$. 
In the current work, we added a small amount (0.5 wt\%) of silicone oil into the 1,2-hexanediol -water binary solution, which forms oil-water microemulsions in the mixture system [93], aiming to utilize silicone oil to trigger the extraction of 1,2-hexanediol. Surprisingly, we observed the plumes of separated 1,2-hexanediol arising along the droplet surface originating from the rim, which resemble those shapes immersing in a Rayleigh-Taylor instability $[94,95]$. To understand the mechanism of the plume formation and the evaporation behaviour of this multicomponent droplet, we studied the drying system experimentally and theoretically.

The paper is organized as follows: In $\S 2$, we introduce the employed experimental methods. In $\S 3$, the experimental results and our interpretations thereof are presented. We then apply multicomponent-diffusion models to the experimentally analyzed cases (section 4 ). The paper ends with a summary and an outlook to the future work (section 5).

\subsection{Experimental methods}

\subsubsection{Solution and substrate}

The droplet system we used consisted of Milli-Q water (Reference A+, Merck Millipore, $25^{\circ} \mathrm{C}$ ), 1,2-hexanediol (Sigma-Aldrich; $\geqslant 98 \%$ ) and silicone oil (SigmaAldrich, viscosity $1000 \mathrm{cSt}$ ). First, $10 \mathrm{wt} \%$ of 1,2-hexanediol aqueous solution was prepared and then $0.5 \mathrm{wt} \%$ of silicone oil was added. For this we mix $30 \mathrm{mg}$ silicone oil with $5970 \mathrm{mg}$ 1,2-hexanediol aqueous solution in a glass container and sonicate it for 10 minutes. We performed evaporation experiments on a hydrophobized glass slide coated by Octadecyltrichlorosilane (OTS, $>90 \%$, Sigma-Aldrich) [80]. Before usage, the substrates were cleaned by 15min sonication in $99.8 \%$ ethanol and 5 min in Milli-Q water sequentially and subsequently dried with compressed $\mathrm{N}_{2}$ flow for $30 \mathrm{sec}$. The droplet in each experiment was deposited by a glass syringe with a full metal needle (Hamilton, $10 \mu \mathrm{L}$, Model 701 NWG SYR, Cemented NDL).

\subsubsection{Confocal microscopy}

Confocal microscopy was employed to visualize the distribution of water and 1,2-hexanediol within the mixture droplet. The observations were carried out by using an inverted Nikon A1 confocal laser scanning microscope system (Nikon Corporation, Tokyo, Japan) with a $10 \times$ dry objective (Nikon, Plan Fluor $10 / 0.30$, OFN25, DIC, L/N1). The droplet was labeled with two 
different dyes, i.e., Nile Red and Dextran. Nile Red is a lipophilic dye which dissolves only in 1,2-hexanediol and was excited by a laser at a wave length of $561 \mathrm{~nm}$, while Dextran preferentially dissolves in water and it was excited by a laser at a wave length of $488 \mathrm{~nm}$ simultaneously. Three-dimensional (3D) images were obtained by reconstruction from a series of consecutive Z-stack images scanned in the direction from the substrate to the top of the droplet. The scan started as soon as the droplet was deposited on the glass substrate. Operating in Galvano mode, the scan rate for the 2D images was $1 \mathrm{fps}$, while each Z-stack scan loop for 3D images took approximately $30 \mathrm{~s}$ to complete. This timescale is much smaller than that of evaporation such that the variation of the flow pattern within the droplet during the scans was negligible.

\subsubsection{Micro particle image velocimetry}

For flow visualization, we performed micro Particle Image Velocimetry ( $\mu \mathrm{PIV})$ by adding fluorescent particles [Fluoro-Max; Red Fluorescent Polymer Microspheres: Ex/Em $530 \mathrm{~nm} / 607 \mathrm{~nm}$; Diameter: $0.52 \mu \mathrm{m}$ ] into the working fluids at a concentration of $2 \times 10^{-2} \mathrm{vol} \%$. The $\mu \mathrm{PIV}$ measurements were implemented on the same confocal microscope with a $10 \times$ dry objective (Nikon, Plan Fluor $10 \times / 0.30$, OFN25, DIC, L/N1). The particles were excited by a laser at a wave length of $561 \mathrm{~nm}$ and the fluorescent signals were captured at a frame rate of $25 \mathrm{fps}$. The droplet was illuminated from the bottom and the fluorescent signal was also captured by the objective from the bottom.

\subsubsection{Geometrical measurement}

The evaporation process was recorded by a CCD camera [MQ013MG-E2, XiQ] coupled to a microscope [12X Ultrazoom, NAVITAR], which was illuminated by LED light [MWWHL4 Warm White Mounted LED, THORLABS] from the opposite side of the droplet. In the experiments, droplets evaporated into air under stable laboratory conditions. The relative humidity $R H$ and the ambient temperature $T$ were monitored in each measurement, which were 45 $\pm 3 \%$ and $22 \pm 1^{\circ} \mathrm{C}$, respectively. 


\subsection{Rayleigh-Taylor instability arising from segre- gation}

\subsubsection{Experimental observations and interpretations}

Evaporation processes of silicone-oil-seeded 1,2-hexanediol-water droplets with opposite orientations are displayed in Fig. 3.1. The upper (a1-a5) and lower (b1-b4) row show the evolution of a sessile droplet and a pendant droplet, respectively. In the beginning of the recording (approximately $30 \mathrm{sec}$ after droplet deposition), the segregation of 1,2-hexanediol already appeared for both droplets [Fig. 3.1(a1,b1)], as revealed by the yellow colour.

For the sessile droplet in Fig. 3.1(a2), in the segregation process, plumes formed, rising from the contact line towards the apex of the droplet. In Fig. 3.1(a3), the shape of the plumes resembles those shapes immersing in a Rayleigh-Taylor instability [96]. The plumes later coalesce with each other, eventually fully covering the whole surface of the droplet and thereby ceasing the evaporation process. However, for the pendant droplet, the separated ring only monotonically grows without any plume formation. This observation clearly demonstrates that the flow structure on the droplet interface is controlled by gravity. The mechanism of the formation of the buoyancy-driven flow structure is interpreted along the schematics in Fig. 3.2. In equilibrium, the silicone oil forms oil-in-water microemulsions owing to the existence of 1,2-hexanediol as a surfactant [93]. After the droplet being deposited on the substrate, some oil droplets nucleate on the solid surface, as was also observed experimentally in Fig. 3.2(c). The reason is that the silicone oil has a much lower interfacial energy with OTS glass than the 1,2-hexanediol-water mixture. This can be seen by comparing the equilibrium contact angle of a pure silicone oil droplet on the OTS glass with that of the 1,2-hexanediol-water mixture droplet. The former is only $10^{\circ}$, the latter is $40^{\circ}$. Early on in the evaporation process, the oil droplets in the oil-water emulsions in the bulk of the droplet aggregate due to the depletion of water. These oil droplets together with those which already nucleated on the substrate trigger the extraction of 1,2-hexanediol from the aqueous solution such that the 1,2-hexanediol phase separates from the water phase.

For a sessile droplet drying on a flat substrate with a contact angle smaller than $90^{\circ}$, the evaporative flux is maximal at the contact line [12]. Hence, the extraction starts from the edge of the droplet due to the fast evaporation of water in that region, leading to an 1,2-hexanediol ring that hinders the further 


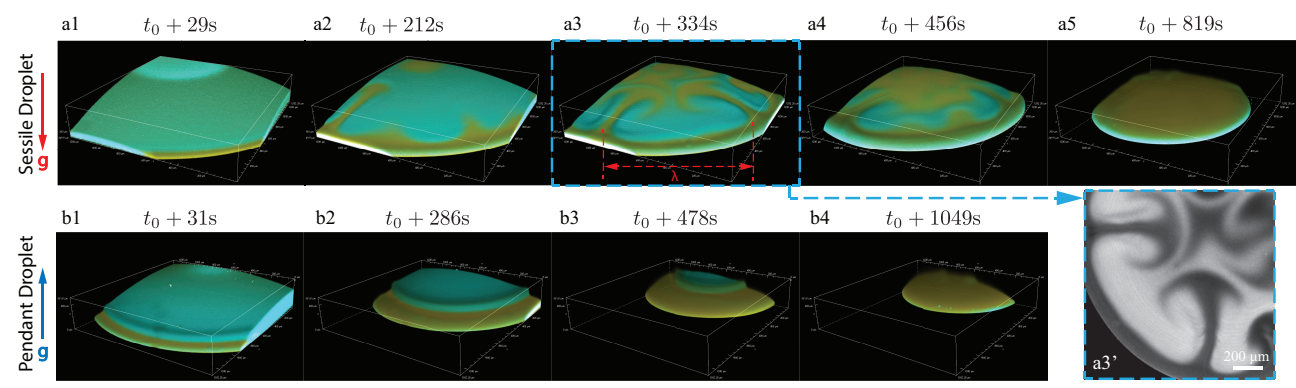

Figure 3.1: Confocal images of evaporation behaviours for both sessile (a1-a5) and pendant (b1-b4) droplets in a semi-side view taken at different time instants. The confocal microscope scanned the rectangular box with the volume $1225 \mu \mathrm{m} \times 1280 \mu \mathrm{m} \times 250 \mu \mathrm{m}$. (a1),(b1) For both droplets, when the evaporation began, the 1,2-hexanediol separated at the contact line and formed a ring-like pattern. (a2-a3) In the sessile droplet, through the growth of the segregation, the separated 1,2-hexanediol rose up with plumes. (a3') The top view of the droplet at $t_{0}+334 \mathrm{~s}$. The figure is transformed into binary image to increase the contrast of colours. (a4) The plumes merged with each other at the apex of the droplet. (a5) Eventually, 1,2-hexanediol fully covered the surface and stopped the evaporation. (b2 - b3) In the pendant droplet, the segregation of 1,2-hexanediol expanded with the ring-like shape. (b4) Once the separated 1,2-hexanediol occupied the entire surface area, the evaporation stopped.

evaporation from the contact line region. The non-volatile ring impedes the building up of the concentration gradient, which results in a suppression of the surface tension gradient, which therefore cannot play any dominant role in controlling the flow. Instead, because of the lower density of 1,2-hexanediol as compared to the mixture, the separated phase of 1,2-hexanediol at the bottom of the droplet rises up to the apex, driven by buoyancy.

\subsubsection{Evidence of suppression of Marangoni flow from $\mu \mathrm{PIV}$ measurements}

To prove our interpretation, we performed $\mu \mathrm{PIV}$ measurements to characterize the flow field within the evaporating silicone-oil-seeded ("SOS") 1,2hexanediol-water droplet. Fig. 3.3(a1) schematically illustrates that the segregation of 1,2-hexanediol suppresses the Marangoni flow in the contact line 


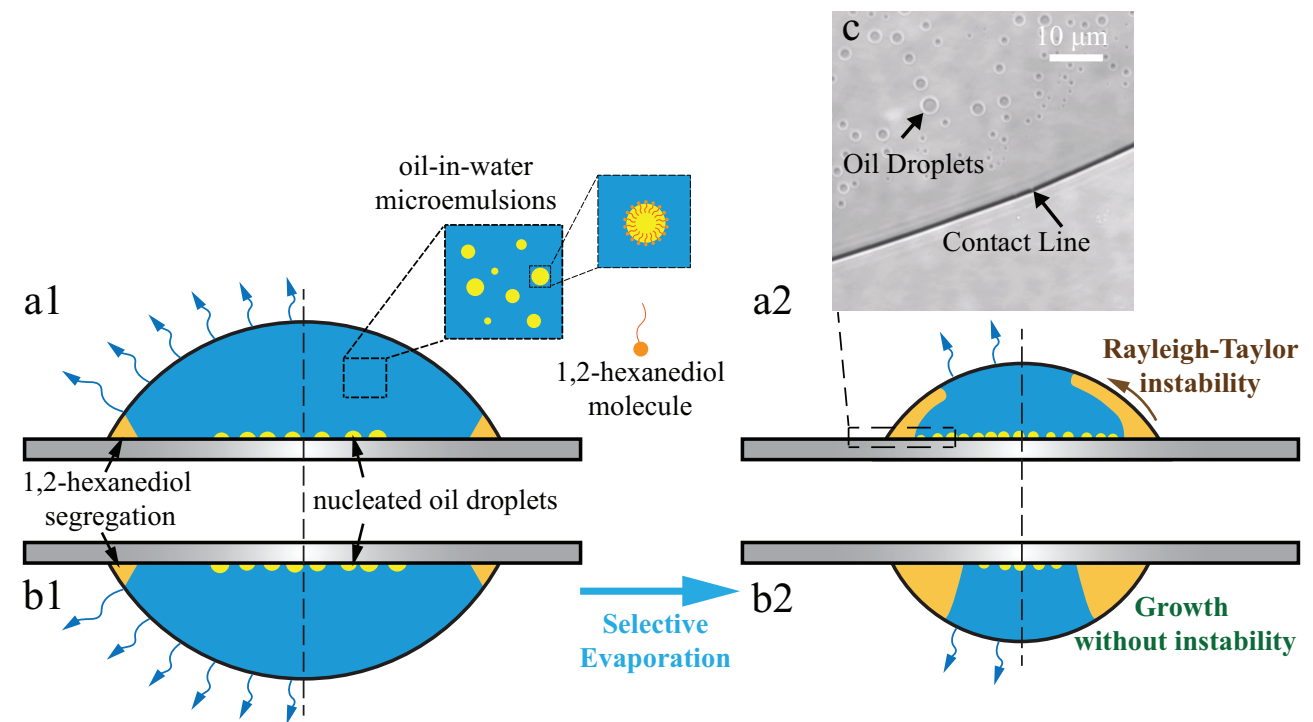

Figure 3.2: Schematics of the silicone-oil-seeded binary droplets with opposite orientations. (a1) Within the bulk of the droplet, there are oil-water microemulsions. Because of the preferential evaporation of water near the contact line, 1,2-hexanediol is extracted by silicone oil and starts separating in this region. The non-volatile 1,2-hexanediol segregation shields the evaporation of water at the rim. (a2) The weak surface tension gradient cannot lead to a strong Marangoni flow on the surface. Instead, in the long term, buoyancy drives the arising plumes (Rayleigh-Taylor instability). (b1) When we orientate the droplet upside down, a similar segregation of 1,2-hexanediol occurs near the contact line. (b2) However, the segregation rim continuously grows due to the selective evaporation of water but no plumes appear due to the inverted direction of gravity. (c) The nucleated oil droplets on the substrate (bottom optical view). 
region. Fig. 3.3(b1) displays a snapshot of the velocity field in the focal plane near the substrate at an early stage of the evaporation process, $t=T_{0} / 30\left(T_{0}\right.$ is the droplet's lifetime). The velocity map shows chaotic and very weak flow motions. Fig. 3.3(c1) shows the evolution of the mean radial velocity and the absolute mean velocity (inlet plot). The mean radial velocity $\bar{U}_{\text {r,sos }}$ is less than 1 $\mu \mathrm{m} / \mathrm{s}$, and the absolute mean velocity $\bar{U}_{\text {sos }}$ in the early stage is around $1 \mu \mathrm{m} / \mathrm{s}$. For comparison, we also measured the flow field for a 1,2-hexanediol-water binary droplet (without the silicone-oil seeding), which reveals the absence of density-driven flow [64]. In that case the velocity map in Fig. 3.3(b2) shows much more intense outward radial flows close to the contact line than that in the case with silicone-oil seeding. During the early lifetime, the mean radial flow velocity $\bar{U}_{\mathrm{r}, \mathrm{no}-\mathrm{sos}}$ [Fig. $3.3(\mathrm{c} 2)$ ] is more than $5 \mu \mathrm{m} / \mathrm{s}$, which is one order of magnitude higher than that in the former case. The measured velocities for both cases imply Reynolds numbers $\operatorname{Re}_{\text {sos }}=\rho R_{d} \bar{U}_{\text {sos }} / \mu \sim 10^{-4}$ and $\operatorname{Re}_{\text {no-sos }}$ $=\rho R_{d} \bar{U}_{\text {no-sos }} / \mu \sim 10^{-3}$, where $\rho \approx 10^{3} \mathrm{~kg} / \mathrm{m}^{3}, R_{d} \approx 10^{-3} \mathrm{~m}$, and $\mu \approx 10$ $\mathrm{mPa} \cdot \mathrm{s}$ are the liquid density, droplet radius, and liquid viscosity, respectively. We estimate the Marangoni time scale in the two cases by using the mean velocity of the radial flow: $t_{\mathrm{Ma} \text {,sos }} \sim R_{d} / \bar{U}_{\mathrm{r}, \mathrm{sos}} \approx 10^{-3} \mathrm{~m} / 10^{-6} \mathrm{~m} / \mathrm{s}=10^{3} \mathrm{~s}$ and $t_{\mathrm{Ma}, \mathrm{no}-\mathrm{sos}} \sim R_{d} / \bar{U}_{\mathrm{r}, \mathrm{no}-\mathrm{sos}} \approx 10^{-3} \mathrm{~m} / 10^{-5} \mathrm{~m} / \mathrm{s}=10^{2} \mathrm{~s}$. By looking at the rising time of the plumes from Fig. 3.1, we obtain the Rayleigh time scale of the RT instability $t_{\mathrm{Ra}} \sim 10^{2} \mathrm{~s}$ (estimated time of the plumes rising up from the rim to the apex of the droplet). In the silicone-oil-seeding case, the Rayleigh time scale is much smaller than the Marangoni time scale: $t_{\mathrm{Ra}} / t_{\mathrm{Ma} \text {,sos }} \ll 1$, which indicates that the buoyancy flow is dominant. In the non-silicone-oil-seeding case, the two time scales are comparable: $t_{\mathrm{Ra}} / t_{\mathrm{Ma}, \mathrm{no}-\mathrm{sos}} \approx 1$, which substantiates that gravity-driven flow is balanced by Marangoni flow, thereby playing no controlling role. We argue that in the silicone-oil-seeded 1,2-hexanediolwater droplet, the instantaneous segregation hinders the evaporation near the contact line, which suppresses the most intensive Marangoni flow in that region, leading to a weak flow motion in the whole droplet. Therefore, on a relatively long time scale, the buoyancy force due to the density difference dominates the flow, causing Rayleigh-Taylor instability.

We estimate the most unstable wavelength of the RT instability in our system $\lambda_{m} \approx 4 \pi\left[\left(\nu^{2} /\left(g_{s} \mathrm{At}\right)\right]^{1 / 3} \approx 10^{3} \mu \mathrm{m}\right.$ [97]. In this expression, $g_{s}=g \sin (\theta)$ is the net acceleration imposed on the interface of the droplet, where $g \approx 9.8 \mathrm{~m} / \mathrm{s}^{2}$ is the gravitational acceleration and $\theta \approx 35^{\circ}$ is the droplet contact angle [see Fig. 3.7(d1,d2)]; At is the Atwood number given by At $=\left(\rho_{m}-\rho_{H}\right) /\left(\rho_{m}+\right.$ $\left.\rho_{H}\right) \approx 2.3 \times 10^{-2}$, where $\rho_{m}=997 \mathrm{~kg} / \mathrm{m}^{3}$ and $\rho_{H}=952 \mathrm{~kg} / \mathrm{m}^{3}$ are the 
density of the mixture and of pure 1,2-hexanediol, respectively [42] and $\nu=$ $\left(\mu_{m}+\mu_{H}\right) /\left(\rho_{m}+\rho_{H}\right)$ is the averaged kinematic viscosity, where $\mu_{m} \approx 2$ $\mathrm{mPas}$ and $\mu_{H} \approx 80 \mathrm{mPas}$ are the dynamic viscosities of the mixture and of pure 1,2-hexanediol, respectively [53]. Here At $\ll 1$, the low density liquid which is 1,2-hexanediol, moves into the heavy fluid in the upper layer [96]. The estimated wavelength $\lambda_{m}$ is comparable to the spatial distance between two plumes $\lambda \approx 10^{3} \mu \mathrm{m}$ in Fig. 3.1(a3), supporting our interpretation of the plumes as RT instability.

\subsubsection{Evaporation-triggered extraction of 1,2-hexanediol by seed- ing oils}

To evaluate the applicability of different oils for the extraction effect, we seeded the 1,2-hexanediol-water binary solution with several kinds of oils at the concentration of $0.5 \mathrm{wt} \%$, namely silicone oils with viscosities of $12500 \mathrm{cSt}$ and 100 cSt. As shown in Fig. 3.4(a,b), the 1,2-hexanediol-water droplets seeded with the three different oils all show similar plumes rising up from the rim of the sessile droplet towards the apex during the evaporation process. The consistency clearly demonstrates that the evaporation can trigger the early extraction of 1,2-hexanediol by the oil-water emulsions in these solutions, leading to the segregation of 1,2-hexanediol and the resulting flow structures.

We also tested a series of concentration ratios of the seeding oil: $0.5 \mathrm{wt} \%$, $0.1 \mathrm{wt} \%, 0.05 \mathrm{wt} \%$, and $0.025 \mathrm{wt} \%$. Our observation shows that the effect holds for the concentration of silicone oil down to $0.025 \mathrm{wt} \%$. The robustness of the extraction effect even at tiny silicone oil concentrations obviously rises the question on the oil-contamination control in such liquid systems. This issue had been addressed before by [98] in a study on nanobubble nucleation. They found that the polydimethylsiloxane (PDMS) coating in a plastic syringe and a disposable needle can contaminate the solution to form nanobubble-like objects on the substrate, which in fact were silicone oil nanodroplets. In their study, the concentration ratio between PDMS (Sylgard 184, Dow Corning) and water was $0.1 \mathrm{~mL} / 0.4 \mathrm{~L}$, which is $\approx 0.025 \mathrm{wt} \%$.

To see whether we can trigger similar contamination effects also here, in a test experiment, we used the same plastic syringe $(5 \mathrm{~mL}$, Discardit, BD) and disposable needle (Microlance, BD) to deposit a 1,2-hexanediol-water binary droplet on the substrate. Indeed, similar RT-like patterns as in our other experiments with silicone oil also occur in such a droplet during the evaporation process [as shown in Fig. 3.4(c)], which confirms that even minute PDMS contamination can cause the early extraction of 1,2-hexanediol during 

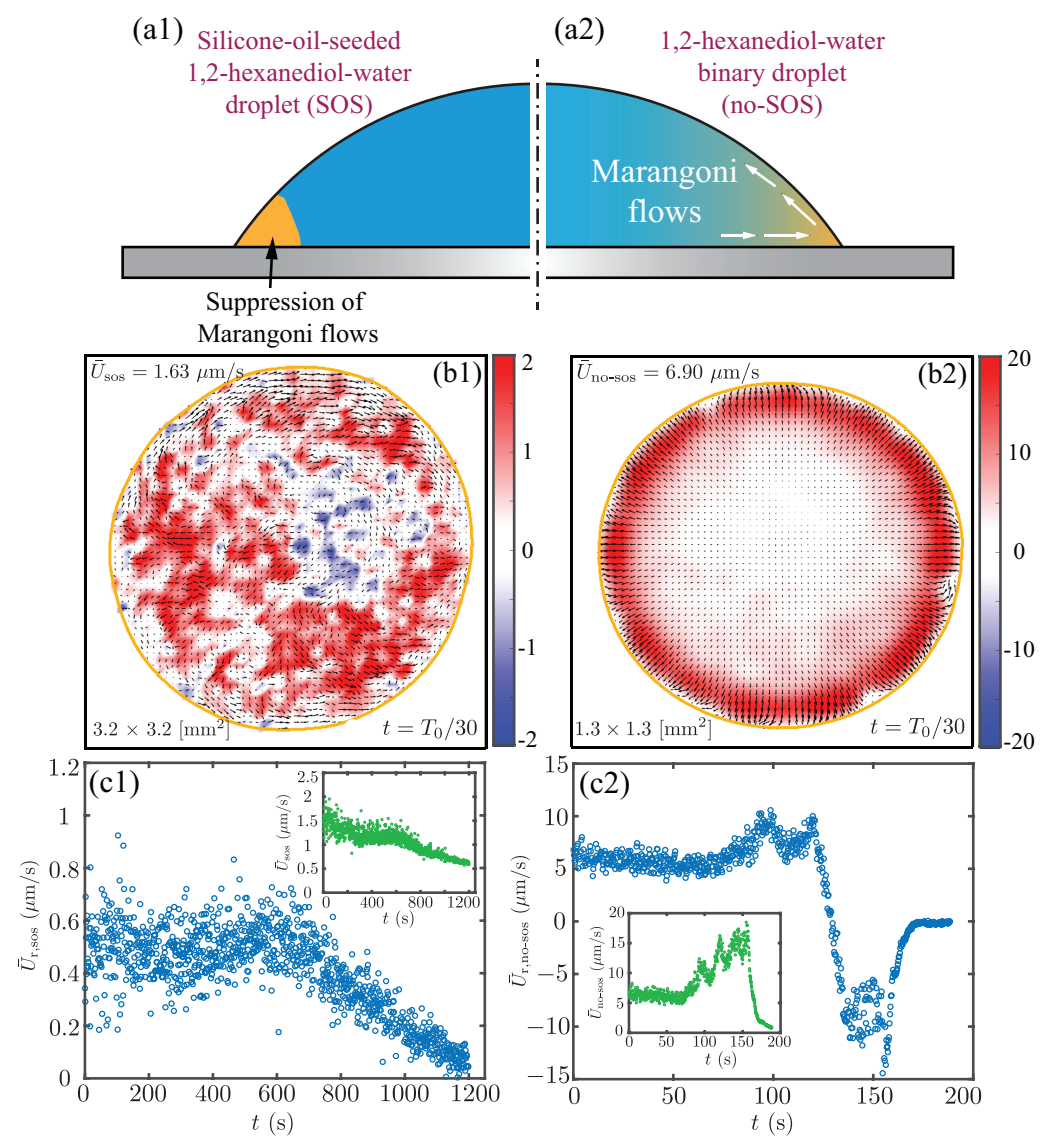

Figure 3.3: $\mu \mathrm{PIV}$ measurements of the velocity fields of a silicone-oil-seeded 1,2-hexanediol-water (SOS) droplet and a 1,2-hexanediol-water (no-SOS) binary droplet [64]. (a1),(a2) Schematics of both droplets at the early life stage. (b1),(b2) $\mu$ PIV snapshot of the velocity field in the focal plane near the substrate at the beginning of the evaporation process. The arrows display the local velocity and the radial velocity is colour coded. (b1) The map shows that there is no visible coherent radial flow. Note that the color scale bar for $U_{\mathrm{r} \text {,sos }}$ ranges from -2 to $+2 \mu \mathrm{m} / \mathrm{s}$, indicating a weak Marangoni flow in a SOS droplet. (b2) The liquid flows radially towards the edge of the no-SOS droplet from the interior. The radial flow is most intense $(\sim 20 \mu \mathrm{m} / \mathrm{s})$ near the contact line, implying a strong Marangoni flow there. The color scale bar for $U_{\mathrm{r}, \mathrm{no}-\mathrm{sos}}$ covers a 10 times larger range as that for $U_{\mathrm{r}, \mathrm{sos}}$. (c1),(c2) The evolution of the radial velocity $U_{\mathrm{r}}$ in the focal plane near the substrate for both droplets. The inlets show the evolution of absolute mean flow velocity $U$. 

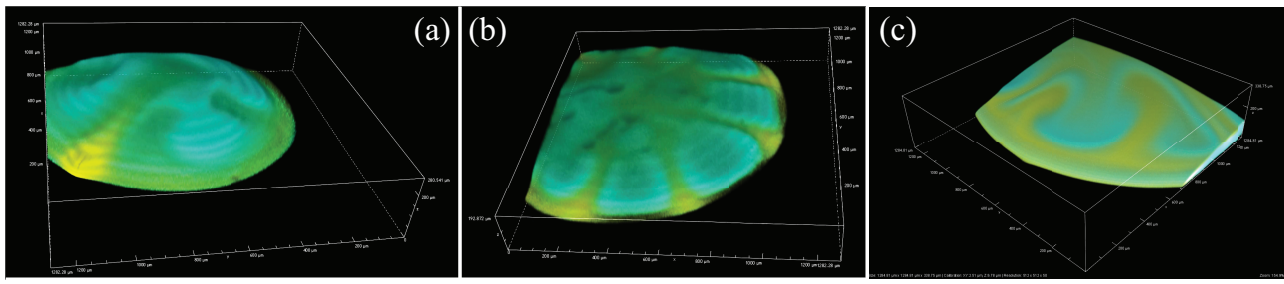

Figure 3.4: Confocal images of segregation patterns for droplets seeded with different oils, namely (a) silicone oil with 12500 cSt viscosity, (b) silicone oil with 100 cSt viscosity, and (c) 1,2-hexanediol-water binary droplet deposited by a plastic syringe and a disposable needle. The confocal microscope scan covered a rectangular box with the section area of $1225 \mu \mathrm{m} \times 1280 \mu \mathrm{m}$.

the evaporation process.

\subsubsection{Reversed segregation by evaporation on a lubricated film}

In the previous sections, we experimentally demonstrated that gravitational effects dominate the flow structure in the droplet system, due to the suppression of Marangoni flow by the instantaneous segregation of 1,2-hexanediol close to the contact line. We argued that the early phase separation is caused by the coupling of the extraction effect by the seeding oil and the maximal evaporation rate at the contact line. In order to validate this argument, we suppress the local evaporative flux at the contact line by introducing a nonvolatile wetting ridge, which can be achieved by letting the droplet evaporate on a lubricated surface $[99,100]$.

We performed the experiment in which the silicone-oil-seeded droplet evaporates on a lubricated surface of silicone oil (Sigma-Aldrich, viscosity $1000 \mathrm{cSt}$ ). The lubricated surface was made by spin coating of silicone oil on a solid glass substrate (Gerhard Menzel GmbH, $76 \times 26 \mathrm{~mm}$ ), with a typical thickness of $18 \pm 1 \mu \mathrm{m}$ [101]. The equilibrium contact angle $\theta$ of the droplet on this lubricated surface is $38^{\circ}$ [see Fig. 3.7 (d1,d2)], which is close to the contact angles in the first two cases. In the experiment, we still only dyed the 1,2-hexanediol and water with the same method mentioned in $\S 2$. Thus the silicone oil film is not visible in the confocal movie.

One wonders whether there is an intercalated film in between the droplet and the substrate. To find out, we performed interferometry [102]. The measurements do not show any interference patterns, which suggests that there is no stable intercalated film. Within a second, the droplet rewets the substrate 

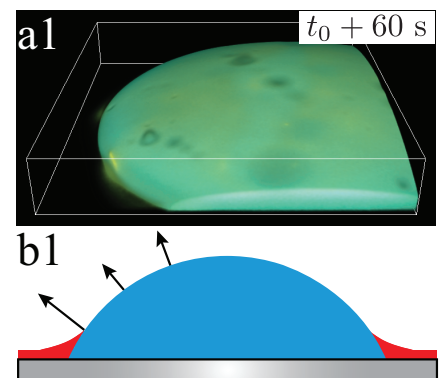

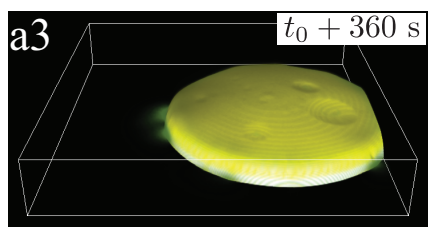

b3

Figure 3.5: The dynamic behaviour of a silicone-oil-seeded 1,2hexanediol/water droplet evaporating on a silicone oil thin film. (a1-a3) Confocal microscopy images for a scanned box with volume $1225 \mu \mathrm{m} \times 1280 \mu \mathrm{m}$ $\times 250 \mu \mathrm{m}$. (a1) In the beginning, the droplet is homogeneously mixed. (a2) The segregation of 1,2-hexanediol started appearing on the upper surface of the droplet instead of contact line area. (a3) The evaporation ceased when the droplet surface was shielded by 1,2-hexanediol. (b1-b3) Schematic of the evaporation process. The red colour represents the silicone oil thin film. It forms a meniscus at the contact line.

by rupturing the thin film after the deposition. We estimate the spreading coefficient $S=\gamma_{\mathrm{da}}-\gamma_{\mathrm{do}}-\gamma_{\mathrm{oa}} \approx 24 \mathrm{mN} / \mathrm{m}-20 \mathrm{mN} / \mathrm{m}-21 \mathrm{mN} / \mathrm{m}<0$, where $\gamma$ is the interfacial tension, and the subscripts a, o, and $\mathrm{d}$ indicate the air and oil phases, and the droplet, respectively. The result $S<0$ is consistent with no oil-engulfment covering on the surface of the droplet. In the beginning of the evaporation [Fig. 3.5(a1)], the droplet is homogeneously mixed, which reveals the green colour. Later on, as shown in Fig. 3.5(a2), the segregation of 1,2-hexanediol (yellow colour) starts appearing at the upper part of the droplet rather than from the edge. Also note that in this third case the segregation behavior is different from the first two cases, as it appears more homogeneously and slowly. The reason is that the segregation in this third case is triggered by the nucleated oil droplets in the bulk instead of those on the substrate, which have a much higher number density in the early phase of the nucleation process and which play the essential role to trigger the early segregation. In Fig. 3.5(a3), the evaporation ceases with the shielding of separated 1,2-hexanediol. This observation shows a different route of segregation, which indicates a faster evaporation rate from the upper surface than from the contact line.

The explanation is that the thin film forms a wetting ridge covering on the 
contact line region, as illustrated in Fig. 3.5(b1), which hinders the evaporative flux from there [100]. Consequently, only the water molecules on the upper surface of the droplet evaporate to the surrounding air, which leads to a high concentration of 1,2-hexanediol at the top of the droplet. Then the highly concentrated 1,2-hexanediol on the upper surface nucleates and segregates from the mixture [Fig. 3.5(b2)]. In the end of the evaporation, there is still water entrapped by the shielding thanks to the segregated 1,2-hexanediol and the silicone oil meniscus, as illustrated in Fig. 3.5(b3).

To further suggest the argument that the wetting ridge suppresses the local evaporative flux at the contact line, we also employed $\mu \mathrm{PIV}$ measurement for the silicone-oil-seeded 1,2-hexnaediol/water droplet evaporating on a silicone oil thin film, as shown in Fig. 3.6. The flow field was measured at the focal plane $\approx 10 \mu \mathrm{m}$ above the substrate. Fig. 3.6(a) displays the bottom-view image at the beginning of the evaporation process. Two circular rings can be observed. As indicated by the black and the yellow arrows, the outer ring and inner ring represent the drop-oil-solid contact line and the drop-oil-air contact line, respectively. Fig. 3.6(b) shows the typical velocity field of the flow structure. The radial velocity $U_{\mathrm{r}}$ is colour-coded. One can see that the radial flow is comparable to the radial velocity in the first case [Fig. 3.3(b1)], which is much weaker as compared to the Marangoni flow in the binary droplet [Fig 3.3(b2)]. Moreover, the outward radial flow from the interior ceases at the horizontal position of drop-oil-air contact line. Near the contact line of the droplet, the radial flow even reverses inwardly [revealed by blue colour in Fig. 3.6(b)]. The flow reversal reflects that the liquid in the contact line region can only flow inwardly to maintain the decreasing contact angle and the pinning contact line at this early stage of the droplet's lifetime [see Fig. 3.7(d1, e1)]. This is a direct evidence that the evaporative flux near the contact line is suppressed by the oil wetting ridge. As shown in Fig. 3.6(c), the mean radial flow decreases and then changes the direction due to the shrinking of the contact area [Fig. 3.7(d1, e1)].

\subsection{Evaporation process and its quantitative under- standing}

A thorough insight in the evaporation process requires a quantitative understanding. Although the evaporation of multicomponent droplet is far more complicated than single-component droplet, the essence of the system is still limited by the diffusion of vapor molecules of each component to the surround- 


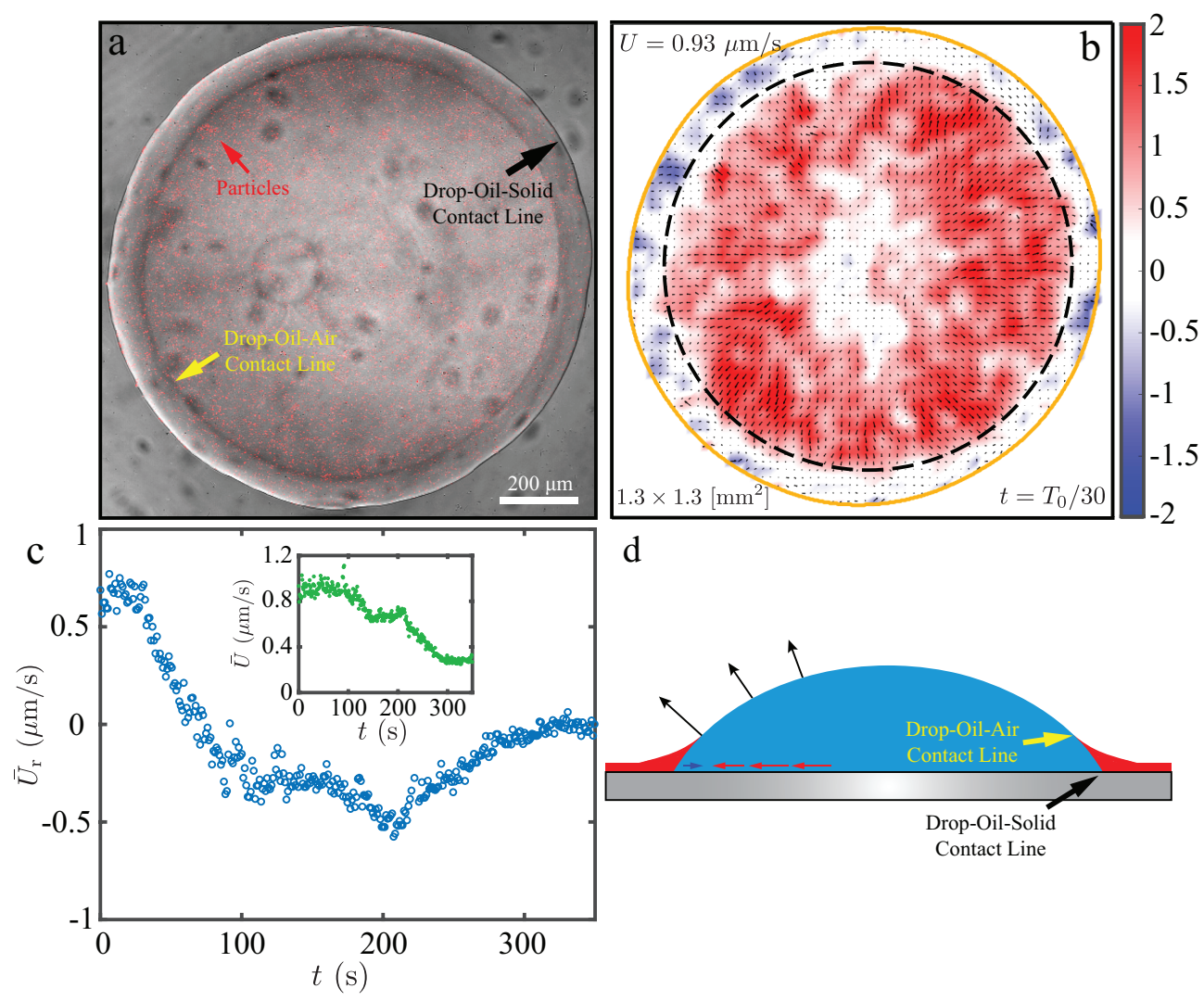

Figure 3.6: $\mu \mathrm{PIV}$ measurement of a silicone-oil-seeded 1,2-hexanediol/water droplet evaporating on a silicone oil thin film. (a) $\mu \mathrm{PIV}$ image focusing on the bottom of the droplet $(\approx 10 \mu \mathrm{m}$ above the substrate). Note that the two circular rings indicate the drop-oil-solid contact line (marked by the black arrow) and the drop-oil-air contact line (marked by the yellow arrow). (b) Flow field of $\mu \mathrm{PIV}$ at $t=T_{0} / 30$, where $T_{0}$ is the droplet's lifetime. The black dashed line indicates the horizontal position of the drop-oil-air contact line. (c) The evolution of the mean radial velocity $\bar{U}_{\mathrm{r}}$ and the mean absolute velocity $\bar{U}$. (d) Schematics of a silicone-oil-seeded 1,2-hexanediol/water droplet evaporating on a silicone oil thin film. 


\subsection{EVAPORATION PROCESS AND ITS QUANTITATIVE UNDERSTANDING59}

ing air $[2,32]$. In this session, we first introduce a diffusion model to describe the evaporative rate for multicomponent droplets. Then we show the geometrical measurement for the three different cases: a. a silicone-oil-seeded 1,2hexanediol-water sessile droplet; b. a silicone-oil-seeded 1,2-hexanediol-water pendant droplet; and c. a silicone-oil-seeded 1,2-hexanediol-water droplet on a lubricated surface. Finally, we compare a modified multicomponent-diffusion model with the influence of segregation considered with our measurement and discuss the results.

\subsubsection{Multicomponent-diffusion model}

[5] proposed an analytical description for the diffusion-controlled evaporation of a sessile droplet with one single component, which was later experimentally confirmed by $[4,103]$. For the evaporation of multicomponent droplets, we use the method put forward by [84], considering the total evaporation rate of the mixture droplet as the sum of the evaporation rates of each individual component. In our droplet mixture system, only the diffusive flux of water contributes to the total evaporation rate. However, non-volatile components in the system also affect the vapor-liquid equilibrium: the existence of 1,2hexanediol and silicone oil change the saturated concentration of water vapor at the liquid-air interface. Raoult's law is used to calculate the difference: $c_{w, s}=X_{w} c_{w, s}^{0}$, where $X_{w}$ is the liquid mole fraction of the water component at the interface and $c_{w, s}^{0}=2.08 \times 10^{-2} \mathrm{~kg} / \mathrm{m}^{3}$ is the saturated vapor concentration of the pure water at room temperature. However, Raoult's law only relies on an ideal solution and thus ignores any interaction between the compositions. To overcome this limitation, a so-called activity $a_{i}$ for each component is introduced to describe this interaction [11], $a_{i}=\psi_{i} X_{i}$, where $\psi_{i}$ is the activity coefficient. In our case, the mole fraction of silicone oil is negligible $(<0.1 \%)$, we only consider the interaction between water and 1,2-hexanediol. Therefore the saturated concentration of water vapor at the interface becomes $c_{w, s}=$ $a_{w} c_{w, s}^{0}$. By using Raoult's law together with the water activity $a_{w}$ [10] to modify the one-liquid model [5], we obtain a theoretical model to express the evaporation rate for the water in our system:

$$
\frac{\mathrm{d} M}{\mathrm{~d} t}=-\pi D R\left(a_{w} c_{w, s}^{0}-c_{w, \infty}\right) f(\theta),
$$

with

$$
f(\theta)=\frac{\sin (\theta)}{1+\cos (\theta)}+4 \int_{0}^{\infty} \frac{1+\cosh (2 \theta \varepsilon)}{\sinh (2 \pi \varepsilon)} \tanh [(\pi-\theta) \varepsilon] \mathrm{d} \varepsilon,
$$


where $D=24.6 \times 10^{-6} \mathrm{~m}^{2} / \mathrm{s}$ is the diffusion coefficient of water vapour in air at room temperature, $R$ and $\theta$ are the footprint radius and contact angle of the droplet, respectively. Besides controlling the evaporation rate, the model also yields the terminal state of the evaporation, which is when the saturated water vapor concentration equals the environmental concentration, $c_{w, s}=c_{w, \infty}$. Theoretically, the evaporation ceases when the active mole fraction of water equals to the relative humidity of the surrounding air, $a_{w}=\psi_{w} X_{w}=R H$. From the relative humidity $R H$ measured in each experiment, we can analytically calculate the "theoretical final volume" $V_{f}$ (as shown in appendix 3.6.1) of each measured droplets as:

$$
V_{f}=\left(\frac{M_{w}}{M_{H}} \frac{R H}{\psi_{w}-R H}+\frac{\rho_{w}}{\rho_{H}}\right)\left(\frac{1-C}{C}+\frac{\rho_{w}}{\rho_{H}}\right)^{-1} V_{0},
$$

in which $M_{H}$ and $M_{w}$ are the molecular mass of 1,2-hexanediol and that of water, $\rho_{H}$ and $\rho_{w}$ are their liquid densities at room temperature, and $C$ is the initial mass concentration of 1,2-hexanediol in each measurement. We rescale the measured droplet volume and time, by introducing non-dimensional volume $\hat{V}=\left(V-V_{f}\right) /\left(V_{0}-V_{f}\right)$ and time $\hat{t}=t / \tau_{c}$ [4], in order to compare the different sets of experimental data. $V$ is the droplet volume measured in every time interval, $V_{0}$ is the initial volume of each measurement and $V_{f}$ is the estimated final volume by Eq. (4.6). $\tau_{c}$ is the characteristic timescale of droplet lifetime, $\tau_{c}=\rho_{l} R^{2} /(D \Delta c)$ [4], where $\rho_{l}$ is the density of the liquid and $\Delta c$ the water vapor concentration difference between the air-liquid interface and the surrounding air.

\subsubsection{Evaporation modes and volumetric measurement}

In Fig. 3.7 we show that the measured contact angle $\theta$ and the footprint radius $R$ of (a) a sessile droplet, (b) a pendant droplet and (c) a droplet on a lubricated surface. Figure. 3.7(a-c) show a snapshot of each droplet. Note that due to the existence of the oil meniscus in the third case [Fig. 3.7(c)], we define the contact angle and footprint radius by fitting the droplet profile with a spherical cap, shown as the yellow dashed line. Figs. 3.7(d-e) show the evolution of the two parameters as a function of the scaled time $\hat{t}=t / \tau_{c}$ and volume $\hat{V}=\left(V-V_{f}\right) /\left(V_{0}-V_{f}\right)$. We observe a sliding contact line during the evaporation for both sessile and pendant droplets. For the sessile droplet, the contact angle remains almost constant during most of the lifetime, but for the pendant droplet, the contact angle always decreases. For the droplet on the thin film, the evaporation follows the stick-slide mode [104-106], i.e., 


\subsection{EVAPORATION PROCESS AND ITS QUANTITATIVE UNDERSTANDING61}

it first evaporates with a pinning contact line, and then shrinks with decreasing contact angle. In Fig. 3.8(a), we show the volumetric evolutions for the three cases. The evaporation lifetime is normally affected by both the evaporation modes and environmental conditions. In Fig. 3.8(b) we compare the volumetric evolution for the three cases by rescaling them with $\hat{t}=t / \tau_{c}$ and $\hat{V}=\left(V-V_{f}\right) /\left(V_{0}-V_{f}\right)$. The deviation between the curves clearly implies that the evaporation kinetics is affected not only by the evaporation modes and environmental conditions, but also by the segregation patterns. Note that the first case with the Rayleigh-Taylor instability [blue squared dots in Fig. 3.8(b)] shows the fastest volumetric decrease among the three cases (compare the slopes of the curves). The percentages of entrapped water compared to the initial amount of water in the final residual of the three droplets were around $16 \%, 30 \%$, and $27 \%$, respectively, estimated from the final volume of droplets. We argue that the fastest evaporation rate coinciding with the lowest water entrapment percentage in the first case is because of the Rayleigh-Taylor instability, causing a better mixing of the components than in the other two cases.

\subsubsection{Evaporative flux profile and modified diffusion model}

To further quantify the mass transfer process, we first use the Sherwood number $S h$ derived by [43] in a diffusion-limited case, and extend it to mixture system by including Raoult's law and activity coefficient :

$$
S h=\frac{\langle\dot{M}\rangle_{A} R_{e q}}{D\left(a_{w} c_{w, s}^{0}-c_{w, \infty}\right)},
$$

where $\langle\dot{M}\rangle_{A}$ is the actual mass loss rate (measured in experiments), averaged over the droplet surface area $A$, and $R_{e q}=(3 V /(2 \pi))^{1 / 3}$ is the equivalent radius. For sessile droplets, if the mass transfer occurs purely via diffusion, the Sherwood number is [43]

$$
S h_{d}=\frac{f(\theta)}{\sin \theta\left(1+\tan ^{2} \frac{\theta}{2}\right)\left(\frac{2}{2-3 \cos \theta+\cos ^{3} \theta}\right)^{1 / 3}},
$$

which only depends on the droplet contact angle $\theta$. Note that the Sherwood number here scales mass transfer with respect to a diffusive spherical droplet. In Fig. 3.9, we plot the experimental data for the three cases by rescaling them based on Eq. (3.4) with an assumption that the mixture liquid components 

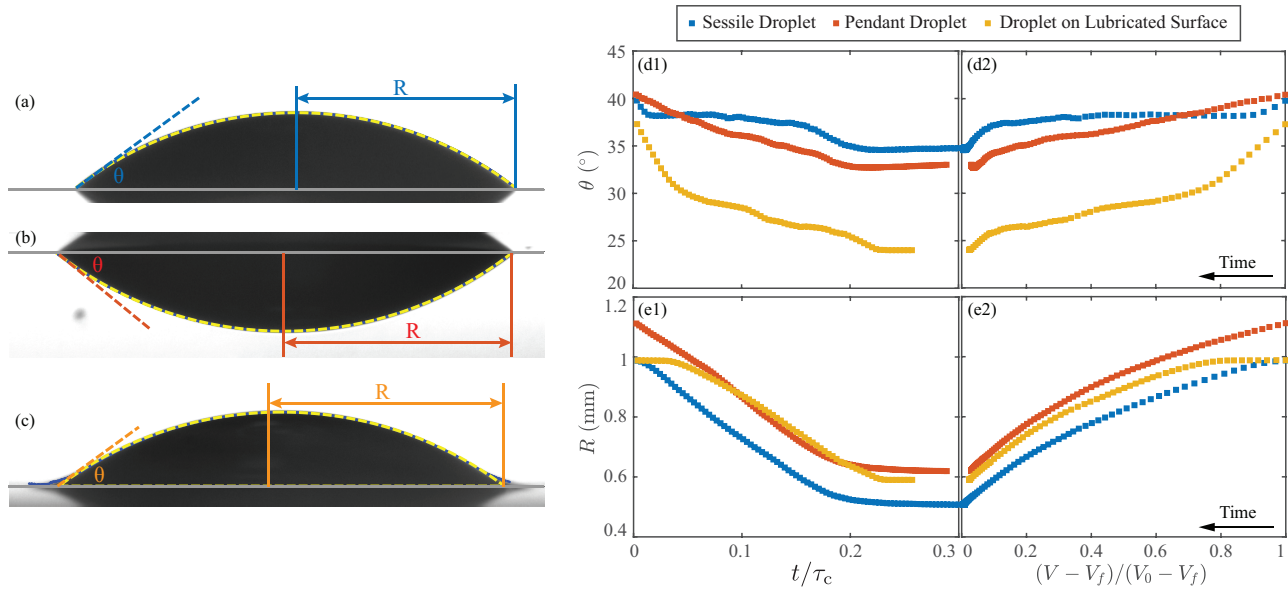

Figure 3.7: Morphology evolution of the evaporating droplets in three different scenarios: sessile, pendant and on the lubricated film. (a-c) Recorded images of the droplets in the three cases, with annotations of the geometrical parameters, i.e., contact angle $\theta$ and footprint radius $R$. Note that for the droplet on lubricated surface (c), we fit the large part of the surface with a spherical curve (see the yellow dashed line), and define the contact angle and footprint radius of the fitting shape as $\theta$ and $R$. (d-e) The contact angle $\theta$ and footprint radius $R$ as a function of scaled time $\hat{t}=t / \tau_{c}$ and scaled droplet volume $\hat{V}=\left(V-V_{f}\right) /\left(V_{0}-V_{f}\right)$.
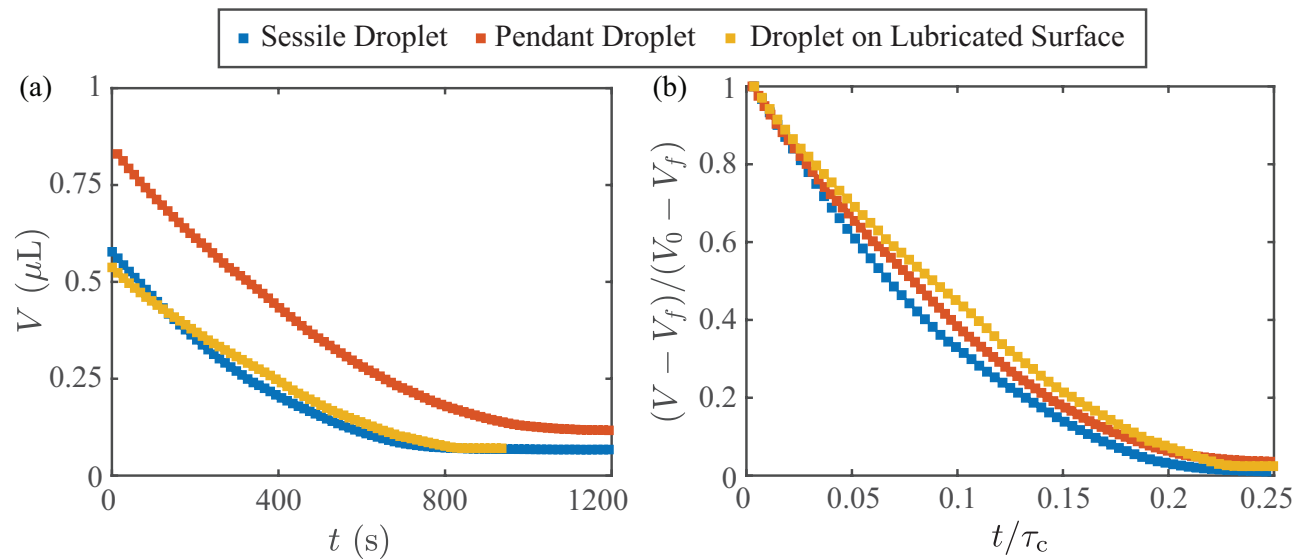

Figure 3.8: (a) Volumetric evolutions of a sessile droplet, a pendant droplet and a droplet on a lubricated surface. (b) Scaled droplet volume $\hat{V}=(V-$ $\left.V_{f}\right) /\left(V_{0}-V_{f}\right)$ as a function of scaled time $\hat{t}=t / \tau_{c}$. 


\subsection{EVAPORATION PROCESS AND ITS QUANTITATIVE UNDERSTANDING63}

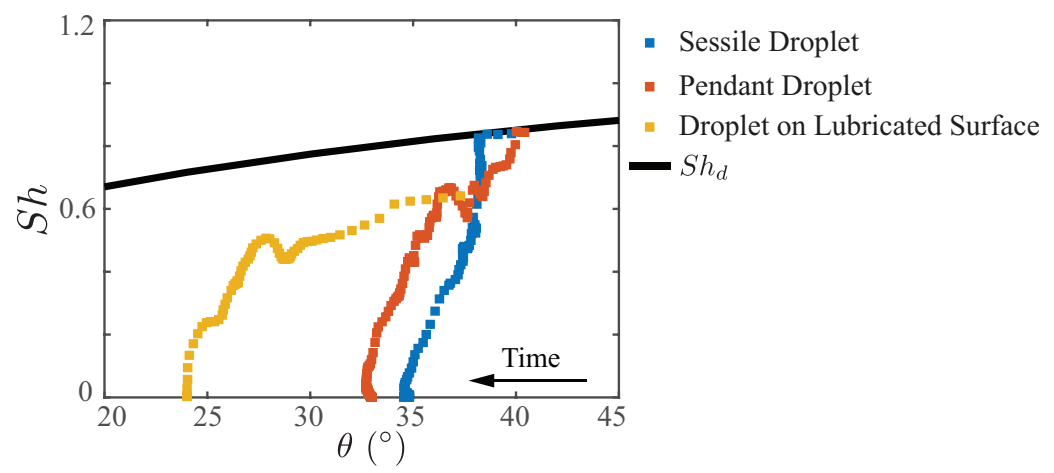

Figure 3.9: Sherwood number as a function of contact angle of a sessile droplet (blue), a pendant droplet (red), and a droplet on a lubricated surface (yellow). The black solid line represents the theoretical Sherwood number $S h_{d}$, which is described by Eq. (3.5).

homogeneously distribute. The overestimation of the Sherwood number following from the single-component diffusive model (black curve) as compared to the experiments indicates that the assumption of homogeneous mixing does not hold for the three cases, which clearly is due to the segregation of 1,2hexanediol. Specifically, for both sessile (blue squared dots) and pendant (red squared dots) droplets, the experimental data and theoretical model show good agreement at the beginning of the evaporation process and they deviate from each other later on. The reason for this deviation is that the distribution of compositions is no longer homogeneous when segregation of 1,2-hexanediol occurs, i.e., the assumption of an evenly mixed system overestimates the local concentration of water on the surface. For the droplet on a lubricated surface (yellow squared dots), the model overpredicts the evaporation rate for the whole process, even from the very beginning. This is due to the fact that the non-volatile oil meniscus hinders the evaporation of water near the contact line during the entire droplet lifetime.

To get a quantitative description of the evaporation rate in the mixture droplets, we further modify the mixture model [Eq. (3.1)] by considering the effect of segregation patterns on the evaporative flux profile of each droplet. For the first two cases, as sketched in Fig. 3.10(a1,a2), the hindrance of the local evaporative flux originates from the non-volatile 1,2-hexanediol segregation. In the limit of small contact angle $\left(\theta<40^{\circ}\right)$, the local evaporative flux on the dropair interface can be described as $j(r, \theta) \sim\left(R_{s}-r\right)^{-\frac{\pi-2 \theta}{2 \pi-2 \theta}}$, which is maximal 

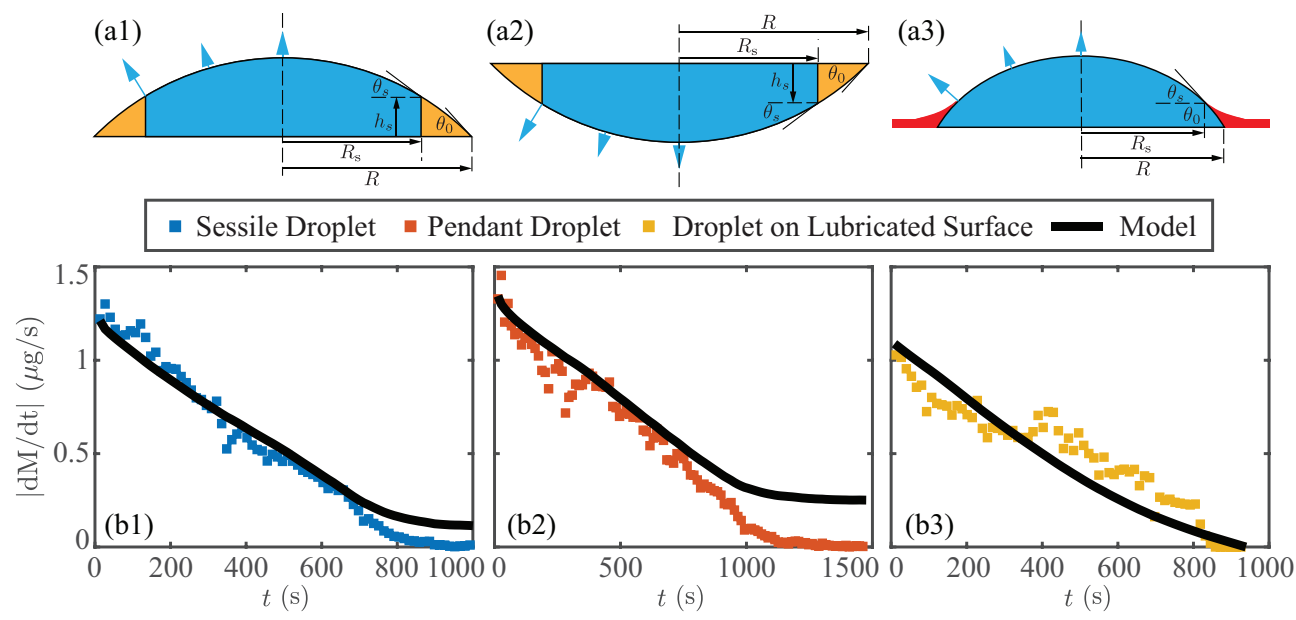

Figure 3.10: Schematic view of the evaporation of (a1) a sessile droplet, (a2) a pendant droplet, and (a3) a droplet on a lubricated surface. (b1-b3) Temporal evaporation rate of each droplet in (a1-a3), respectively, during the evaporation process. The black solid line represents the prediction of the theoretical model.

at $r=R_{s}[12,107] . R_{s}$ is the distance between the center of the droplet and the front of the segregation. Hence we obtain the total evaporative flux of the droplet with segregation by replacing $R$ with $R_{s}$ in Eq. (3.1):

$$
\frac{\mathrm{d} M}{\mathrm{~d} t}=\int_{0}^{R_{s}} j(r, \theta) \sqrt{1+\left(\partial_{r} h\right)^{2}} 2 \pi r d r=-\pi D R_{s}\left(a_{w} c_{w, s}^{0}-c_{w, \infty}\right) f\left(\theta_{s}\right),
$$

where $\theta_{s}$ is the tangential angle at the front of the segregation [see Fig. 3.10(a1)]. As the angle is small, $0 \leq \theta_{s} \leq 40^{\circ}$, we approximate $f\left(\theta_{s}\right) \approx 4 / \pi[15,103]$ in our calculation for convenience. This approximation was shown to be appropriate in colloidal suspension droplets with similar configurations [107]. In order to calculate $R_{s}$, the volume of the segregated liquid is required. From the confocal imaging, we observe an almost instantaneous segregation of 1,2hexanediol. Hence we assume that the water mostly depletes in the segregation region [the yellow part in Fig. 3.10(a1)] and remains nearly constant in the mixture region [the blue part in Fig. 3.10(a1)]. The mass in the segregation region $\Delta M_{s}$ can be calculated by multiplying the volume of the region $\Delta V_{s}$ and the density of 1,2-hexanediol $\rho_{H}$ : 


$$
\Delta M_{s}=\rho_{H} \Delta V_{s}=\rho_{H} \int_{R_{s}}^{R} 2 \pi r h(r) d r=\frac{\pi \theta}{4 R}\left(R^{2}-R_{s}^{2}\right)^{2},
$$

where $h(r)=\frac{R^{2}-r^{2}}{2 R} \theta$ is the local height calculated from the parabolic approximation at distance $r$ from the center of the droplet. The separated 1,2hexanediol is caused by the depletion of water $\Delta M_{w}=\rho_{w} \Delta V$, where $\Delta V$ is the volume loss measured from the experiment and $\rho_{w}$ is the density of water. According to the initial mass concentration of 1,2-hexanediol $C$, we then have:

$$
\Delta M_{s}=\frac{C}{1-C} \Delta M_{w}=\frac{C}{1-C} \rho_{w} \Delta V .
$$

By combining Eq. (3.7) and Eq. (3.8), we obtain

$$
R_{s}=R\left(1-\sqrt{\Delta V \frac{C}{1-C} \frac{\rho_{w}}{\rho_{H}} \frac{4}{\pi \theta R^{3}}}\right)^{1 / 2} .
$$

By substituting Eq. (3.9) into Eq. (3.6), we can theoretically calculate the evaporation rate without any adjustable parameters. Note that we approximate $a_{w}\left(X_{w}\right)$ by taking the initial water mole fraction $X_{w}$ as a constant for the whole process. For the third case where the droplet evaporates on the thin film, the evaporative flux profile is changed by the hindrance of the oil wetting ridge. As shown in Fig. 3.10(a3), the evaporative flux profile is identical to the first two cases: it is maximal at the drop-oil-air contact line. Therefore, the total evaporation flux rate can also be described by $\mathrm{Eq}(3.6) . R_{s}$ then is the horizontal distance from the center of the droplet to the triplephase contact line. We can measure $R_{s}$ by determining the position of the triple-phase contact line from the bottom-view image, as shown in Fig. 3.6(a). We use $a_{w}\left(X_{w}\right)$ with a temporal water mole fraction $X_{w}$ calculated from the volumetric measurement.

In Fig. 3.10(b1-b3), we show the measured evaporation rate corresponding to each case and compare them with the theoretical prediction by Eq. (3.6). For both sessile and pendant droplets [Fig. 3.10(b1-b2)], the experimental data and theoretical model show good agreement for a large part of the process and deviate from each other only in the late stage of the lifetime. The main reason for this deviation at the very end of the evaporation process is that the entrapped water by the segregation of 1,2-hexanediol does not homogeneously distribute in the droplet, i.e., the assumption of an evenly mixed system overestimates the local concentration of water on the surface. For the droplet on a lubricated surface, the model gives a good description of the evaporation rate. 
Finally note that in the second half of the lifetime, the model underestimates the evaporation rate. This can be explained by the fact that near the triplephase contact line, the wetting edge is not thick enough to completely hinder the evaporation of water. Water can still evaporate into air in that region by first diffusing through the thin layer of oil.

\subsection{Summary and outlook}

In this work, we experimentally studied the evaporation behaviour of a siliconeoil-seeded 1,2-hexanediol-water droplet. The observation shows an instantaneous segregation of 1,2-hexanediol in the sessile droplet followed by the formation of plumes of the segregated fluid. By orientating the droplet upside down, the absence of the plumes indicates that the observed instability is indeed controlled by gravity, confirming the interpretation as Rayleigh-Taylor instability. We have shown that through hindering the strong evaporation near the contact line by segregation of non-volatile 1,2-hexanediol, the Marangoni effect can be significantly suppressed, and thus allowing buoyancy force to play a dominant role in the flow structure. Following the idea of suppressing evaporation locally by coverage with a non-volatile component, we can manipulate the segregation to start preferentially from the top rather than the edge of the droplet by letting the droplet evaporate on a lubricated surface.

Since the evaporation of droplets shows complex physicochemical behaviour, i.e., segregation of 1,2-hexanediol, it is difficult to obtain knowledge of the local concentration. We theoretically apply a scaling analysis on the experimental investigation of droplets in three cases, namely the sessile droplet, the pendant droplet and the droplet on a lubricated surface. It shows different evaporation modes of contact line behaviour in each case. By comparing the measurement of the evaporation rate with the multicomponent-diffusion model, we show that the segregation of the non-volatile component significantly delays the evaporation of water and, even leading to entrapment of water in the residue of the droplets.

To our best knowledge, this work is the first observation of the Rayleigh-Taylor instability in a microdroplet system triggered by evaporation. It is another example that the Rayleigh convection can overcome Marangoni effects to control the flow structure in a milli-sized droplet with Bond number $<1$. As we have demonstrated that the mixing effect is highly influenced by the flow pattern, our finding can be crucial for many applications involving uniform surface coating and particle assembly. We have also shown that such a surprising 
phenomenon can be triggered by various different seeding oils, even at very low concentrations. We think that it is important for the community to realize that during evaporation processes which involve several different components, each component even with minute amount may dramatically influence the overall behavior.

Moreover, droplets need not always evaporate on a solid surface: There are many applications involving droplets drying on lubricated surfaces. In particular, in the inkjet printing process, a primary layer is printed on surfaces prior to the deposition of ink drops, in order to destabilize the pigments to improve the printing quality [101]. Our study of a silicone-oil-seeded mixture droplet evaporating on a lubricated surface provides an effective way to manipulate the segregation in such drying systems by utilizing the non-volatile meniscus to impede the evaporation from the edge.

Many questions still remain open. The difficulty to accurately predict the droplet lifetime originates from the lack of means to accurately monitor the local distribution of the liquid components. The complexity arises not only from the segregation of 1,2-hexanediol, but also the later plume formation. How to predict the onset of the Rayleigh-Taylor instability in this geometry of spherical cap? Does the unstable wave length between the plumes depend on the size or the contact angle of the droplets? To answer these questions, further studies on the parameter space are worthwhile. By considering the limitation of experimental measurement, a detailed insight may even require numerical simulations.

\subsection{Appendix}

\subsubsection{Derivation of theoretical final volume}

The molar fraction of water in binary mixture is defined as the number of moles of water divided by the number of moles of both liquids. Here we neglect the tiny mole fraction $(<0.1 \%)$ of silicone oil. The mole fraction of water is

$$
x_{w}=\frac{m_{w} / M_{w}}{m_{w} / M_{w}+m_{H} / M_{H}}=\frac{1}{1+\frac{m_{H}}{m_{w}} \frac{M_{w}}{M_{H}}} .
$$

where $m_{w}$ is the mass of water and $m_{H}$ is the mass of 1,2-hexanediol. Here we assume that the density of the mixture is linear with the solute concentration, which means that the total volume of the mixture is the sum of the individ- 
ual liquid volumes [108]. This is a reasonable approximation for water/1,2hexanediol mixture. Then the initial density is given by

$$
\rho_{i}=\frac{m_{H}+m_{w}}{\frac{m_{H}}{\rho_{H}}+\frac{m_{w}}{\rho_{w}}}=\frac{\left(m_{H}+m_{w}\right) \rho_{H} \rho_{w}}{m_{H} \rho_{w}+m_{w} \rho_{H}},
$$

where $\rho_{i}$ is the initial density of the mixture, $\rho_{H}$ and $\rho_{w}$ are the density of 1,2hexanediol and water, respectively. We introduce the initial mass percentage $C$ of the solute, whose value can be between 0 and 1 . Then we have $m_{w}=$ $m_{H} \frac{1-C}{C}$ at the beginning before evaporation. By substituting it into Eq. 4.10, the equation reduces to

$$
\rho_{i}=\frac{\left(m_{H}+m_{H} \frac{1-C}{C}\right) \rho_{H} \rho_{w}}{m_{H} \rho_{w}+m_{H} \frac{1-C}{C} \rho_{H}}=\frac{\rho_{H} \rho_{w}}{\rho_{H}+C\left(\rho_{w}-\rho_{H}\right)} .
$$

The 1,2-hexanediol mass in the droplet is now given by $V_{i} \rho_{i} C$, which is constant during the drying process due to the non-volatility of 1,2-hexanediol. $V_{i}$ is defined as initial droplet volume. Therefore the amount of water that has evaporated is given by $\left(V_{i}-V\right) \rho_{w}$, and the total mass of water left in the droplet is the initial mass minus the evaporated mass, $V_{i} \rho_{i}(1-C)-\left(V_{i}-V\right) \rho_{w}$. By substituting the water mass into Eq. 4.9, the mole fraction of water in the droplet is expressed as

$$
x_{w}=\frac{1}{1+\frac{V_{i} \rho_{i} C}{V_{i} \rho_{i}(1-C)-\left(V_{i}-V\right) \rho_{w}} \frac{M_{w}}{M_{H}}} .
$$

From the theory, it is predicted that the droplet stops evaporating at the moment when the active mole fraction of water equals to relative humidity of surrounding air, $a_{w}=\psi_{w} x_{w}=R H$. Then we obtain the theoretical final mole fraction $x_{w}=R H / \psi_{w}$, where $R H$ and $\psi_{w}$ is the relative humidity and activity coefficient of water [57], respectively. By substituting $x_{w}$ into Eq. 4.12, we can analytically calculate the theoretical final volume $V_{t}$ :

$$
V_{f}=\left(\frac{M_{w}}{M_{H}} \frac{R H}{\psi_{w}-R H}+\frac{\rho_{w}}{\rho_{H}}\right)\left(\frac{1-C}{C}+\frac{\rho_{w}}{\rho_{H}}\right)^{-1} V_{0} .
$$




\section{Chapter 4}

\section{Evaporation-induced crystallization of surfactants in sessile multicomponent droplets*}

Surfactants have been widely studied and used in controlling droplet evaporation. In this work, we observe and study the crystallization of sodium dodecyl sulfate (SDS) within an evaporating glycerol-water mixture droplet. The crystallization is induced by the preferential evaporation of water, which decreases the solubility of SDS in the mixture. As a consequence, the crystals shield the droplet surface and cease the evaporation. The universality of the evaporation characteristics for a range of droplet sizes is revealed by applying a diffusion model, extended by Raoult's law. To describe the nucleation and growth of the crystals, we employ the 2-dimensional crystallization model of Weinberg, J. Non-Cryst. Solids, 1991, 134, 116. The results of this model compare favorably to our experimental results. Our findings may inspire the community to reconsider the role of high concentration of surfactants in multicomponent evaporation system.

*Based on: Yaxing Li, Valentin Salvator, Herman Wijshoff, Michel Versluis and Detlef Lohse, Evaporation-induced crystallization of surfactants in sessile multicomponent droplets. Langmuir (2020) (In press). 



\subsection{Introduction}

Surfactants are widely used to control the evaporation behavior of sessile droplets on a flat substrate [109-111]. The motivation is driven by various applications in inkjet printing, surface coating and patterning [25, 58], which mainly aim to optimize the drying rate and the final deposition. The biggest challenge for a controlled uniform coating by droplet evaporation originates from the well-known "coffee-stain effect" [12]. It has been shown that surfactant-induced Marangoni flow can play an essential role to suppress this effect $[31,112]$. In these studies, one of the most common ionic surfactants, "sodium dodecyl sulfate" (SDS) [113-115] is added to the system at small concentration, typically $\leq 1 \mathrm{wt} \%$. The surfactants are therefore considered to be always soluble in the system during most of the evaporation lifetime. However, in many practical cases, the relevant liquids contain a high concentration of surfactants; e.g., liquid detergents can contain surfactant ingredients at up to $40 \%$ by weight. Such high loading of surfactants may lead to undesired effects, such as separation and crystallization.

Sodium dodecyl sulfate (SDS) may crystallize in liquid solutions upon cooling [116] or upon seeding with 1-dodecanol [117]. On the other hand, selective evaporation of some liquid components with larger volatilities can also lead to phase separation in multicomponent mixtures $[49,64,88]$. Consequently, the nonvolatile surfactant (SDS) is expected to separate from an evaporating liquid system by crystallization due to the preferential evaporation of volatile liquids. Therefore, the wide-usage of SDS in evaporating droplet systems deserves a more detailed explanation of the crystallization behaviour.

In this work, we study a multicomponent droplet system consisting of a mixture of glycerol, water, and SDS and let it evaporate in ambient air. SDS is not miscible with pure glycerol, but it does dissolve in glycerol-water mixtures for large enough water concentration ratios. This behavior qualitatively resembles the ternary "ouzo" system [118] consisting of water, ethanol, and anise oil, which nucleates in droplets for low enough ethanol concentrations. Tan et al. $[24,47]$ triggered this emulsification threshold by the selective evaporation of ethanol in an evaporating ouzo droplet. Similarly, the varying solubility of SDS in glycerol-water binary systems may also lead to phase separation due to the concentration change caused by the selective evaporation of water alone. In contrast to crystallization by cooling $[116,117]$, here the oversaturation with SDS and the subsequent nucleation and growth of SDS crystals is caused by the preferential evaporation of water at room temperature [119]. 
To better understand the evaporation-induced crystallization in the mixture droplet system, two main questions need to be addressed: how does a surfactantladen mixture droplet evaporate and how to model the crystallization during the evaporation? In this paper we want to answer these questions. A typical snapshot of an evaporating droplet is shown in Fig. 4.2, where the two life phases can be distinguished: the evaporation phase and the crystallization phase. The focus of our study is on the dynamics of the evaporation and the kinetics of crystallization, and not on the micro-scale crystal morphology.

\subsection{Experimental Methods}

\subsubsection{Materials and preparation}

The liquid solution was prepared with an initial composition of $78 \%(\mathrm{w} / \mathrm{w})$ Milli-Q water (Reference A+, Merck Millipore, 25), 19.6\% (w/w) glycerol (Sigma Aldrich; purity $\geq 98$ ) and $2.4 \%(\mathrm{w} / \mathrm{w})$ sodium dodecyl sulfate (Sigma Aldrich, purity 98\%). The initial concentration of SDS is 13 CMC (critical micelle concentration). Experiments were carried out on a transparent hydrophobic octadecyltrichlorosilane (OTS)-glass substrate [80]. The static contact angle of Milli-Q water and glycerol on the substrate are $105^{\circ} \pm 3^{\circ}$ and $90^{\circ} \pm 3^{\circ}$, respectively. The glycerol-water binary droplet with 50\%/50\% $(\mathrm{w} / \mathrm{w})$ has a $95^{\circ}$ static contact angle. Prior to each experiment, the samples were cleaned by sonication in an ultrasonic bath of ethanol and subsequently in water, then dried under a flow of nitrogen gas.

\subsubsection{Experimental setup}

We performed two different experiments to separately study the evaporation phase and crystallization phase. To study the evaporation behavior, the droplets were deposited on the substrate by a Hamilton $2 \mu \mathrm{L}$ syringe, which was mounted vertically on a computer-controlled motorized pump, that allowed the dispense of droplets of a controlled volume through a needle. We measured the geometry of the deposited droplet by bright-field imaging in side view. The whole process was recorded by an OCA 15 (Dataphysics, Germany) contact angle device (Fig. 5.6.a): a CCD camera coupled to a microscope, which was back-illuminated by a LED light from the opposite side of the droplet. For the crystallization study, we observed the droplet in bottom view with a confocal microscope (Fig. 5.6.b). By focusing on the layer close to the substrate (at $\mathrm{a} \approx 10 \mu \mathrm{m}$ height), the dynamic growth of the crystals 
was visualized in a 2-dimensional view. The experiments were performed at room temperature of $21.4 \pm 1{ }^{\circ} \mathrm{C}$ and at relative humidity of $50 \% \pm 5 \%$. These parameters were monitored and recorded for each measurement.
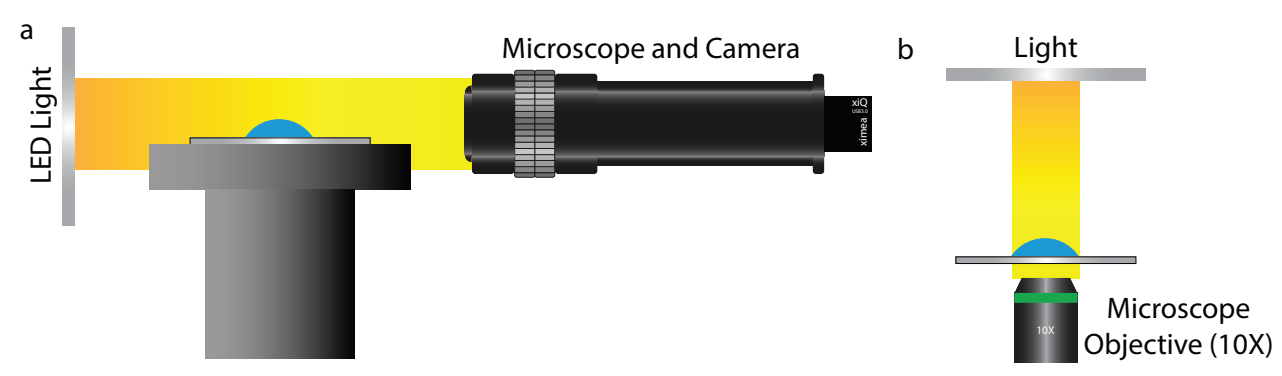

Figure 4.1: A schematic sketch of the experimental setups. (a) The contact angle device contains a CCD camera with a microscope and a LED light source illuminating the droplet. (b) The droplet is illuminated from above and recorded by a camera equipped with a $10 \times$ microscope objective underneath. The whole setup is part of a confocal microscope (Nikon A1 confocal laser microscope system, Nikon Corporation, Tokyo, Japan).

\subsubsection{Imaging analysis}

For the side-view geometrical measurement, images were analyzed using a custom-made Matlab code to detect the droplet profile with sub-pixel accuracy [50]. The sizes of all droplets are smaller than the capillary length $\sqrt{\gamma /(\rho g)} \approx 2.7 \mathrm{~mm}$ for the used liquids [16], where $\gamma \approx 70 \mathrm{mN} / \mathrm{m}$ and $\rho \approx 10^{3}$ $\mathrm{kg} / \mathrm{m}^{3}$ are the surface tension and density of the mixture, and $g=9.8 \mathrm{~m} / \mathrm{s}^{2}$ is the gravitational acceleration. The detected profile is fitted to a spherical cap during the evaporation phase, which enables us to calculate the volume $V$ of the droplet with footprint radius $R$ and contact angle $\theta$. As shown in Fig. 4.2A, the dark blue solid line is the position of the substrate: the spherical shape above it is the sessile droplet, the one underneath is its reflection.

For the documentation of the crystallization process from the bottom view, a manual detection with ImageJ was used to measure the crystallized area at every time instant, see details in Supplementary Materials. 


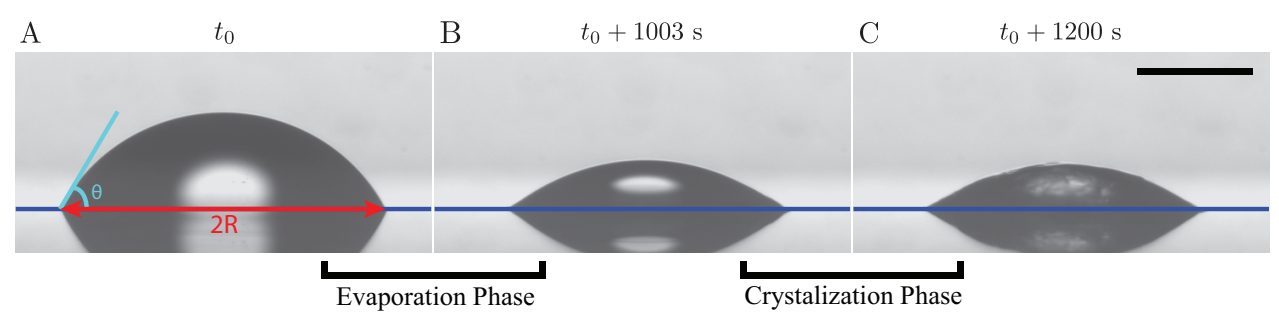

Figure 4.2: Experimental snapshots of the evaporation and drying process of a typical drop on a flat surface. (A) and (B) show the evaporation phase of the drop: here, the drop retains a spherical cap shape; no crystallization occurs. (C) The final state of the drop: due to the crystallization of SDS in the bulk, the surface of the drop buckles and no longer remains spherical. The crystallization of the SDS shields the surface and brings the evaporation process to an end. The scale bar represents $0.5 \mathrm{~mm}$.

\subsection{Experimental results}

\subsubsection{Evaporation phase}

The left column of Fig. 4.3 displays the temporal evolution of the dropcharacterizing geometrical parameters for four droplets with different initial sizes : volume $V$ (A1), contact angle $\theta$ (B1), and footprint radius $R(\mathrm{C} 1)$. From the plots, it is evident that all the droplets evaporate following the "stick-slide" mode $[9,104]$, in which the droplet's footprint radius first remains constant until it reaches a critical contact angle, then the contact line starts to shrink. We only measure the volume until buckling occurs (as marked by the red circles in Fig. 4.3A1, C1), and after that, the droplet shape deforms and no regular shape is reestablished, which from then on renders accurate volume measurement impossible. Fig. 4.4A shows the average evaporation rate of various droplets in the first $30 \mathrm{sec}$ after deposition with initial volumes ranging from $0.12 \mu \mathrm{L}$ to $2.40 \mu \mathrm{L}$. The evaporation rate monotonically increases with increasing droplet size, apart from fluctuations due to experimental uncertainties.

\subsubsection{Crystallization phase}

Figure 4.5 shows the complete crystallization process of an evaporating surfactantladen mixture droplet. The droplet starts to evaporate at time $t_{0}$. At approximately $50 \mathrm{sec}$, the first crystals appear near the contact line (CL) region. After 

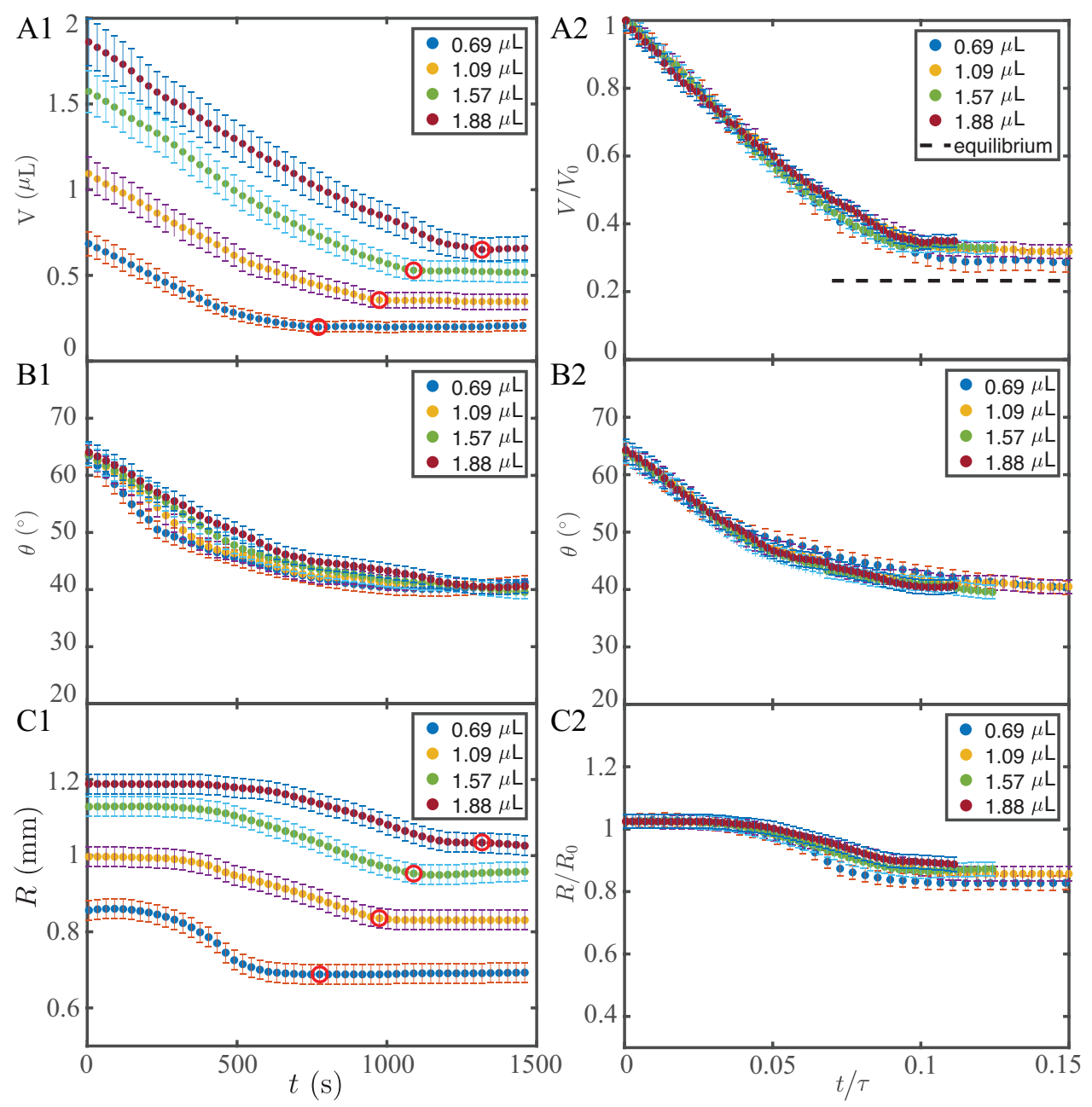

Figure 4.3: (A1,B1,C1) Measured temporal evolution of the geometrical parameters: volume $V(\mathrm{~A} 1)$, contact angles $\theta$ (B1)and lateral sizes $R(\mathrm{C} 1)$. The red dots mark the moments when buckling occured. (A2,B2,C2) Same parameters as in experiment, but now non-dimensional and plotted against the scaled time following Eq. (4.4). The data collapse clearly shows the universality of the drop evaporation process. (A2) The final volume is controlled by the occurrence of crystalization, rather than by the liquid-vapor equilibrium relation, which is shown by the black dashed line. 

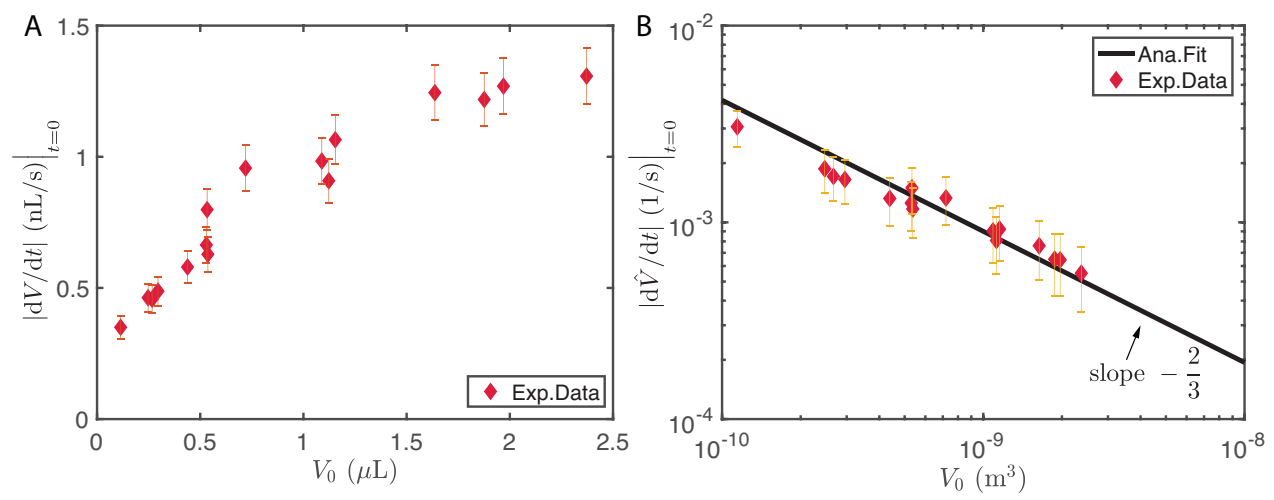

Figure 4.4: (A) The initial rate of volume loss of the drop varies for different initial volumes. (B) The same data normalized by the initial volume is plotted against the initial volume. The straight line shows the scaling relation with slope $-2 / 3$, demonstrating good agreement with the experimental data.

a few more seconds, several crystals nucleate at the rim. Then they grow and coalesce to form a larger piece and finally occupy the whole bulk of the droplet. Figure 4.6 presents a zoomed-in bottom view of the contact region of another evaporating surfactant-laden mixture droplet. Initially, the droplet is transparent with a smooth CL. After evaporating for $280 \mathrm{sec}$, a crystal nucleates near the CL and floats to the position labeled by the yellow circle. A few seconds later, more crystals nucleate at the rim, slightly deforming the CL. The nucleated crystals grow and coalesce with neighbouring crystals. Eventually, the whole droplet is occupied by the crystals and the CL deforms and is no longer smooth. Figure 4.9A shows the temporal evolution of the transformed fraction measured in a 2D bottom view for three different droplets. $X$ is the area fraction occupied by crystals and $t$ is the time which has elapsed after the first crystallization had been observed. The area fraction $X$ increases as the growth of crystals at a different rate for each droplet.

\subsection{Theoretical analysis}

\subsubsection{Theory of mixture droplet evaporation}

We first study the evaporation characteristics of the surfactant-laden mixture droplet. In general, for a droplet evaporating on a flat surface under ambient conditions and in the absence of any correction, the evaporation is fully con- 


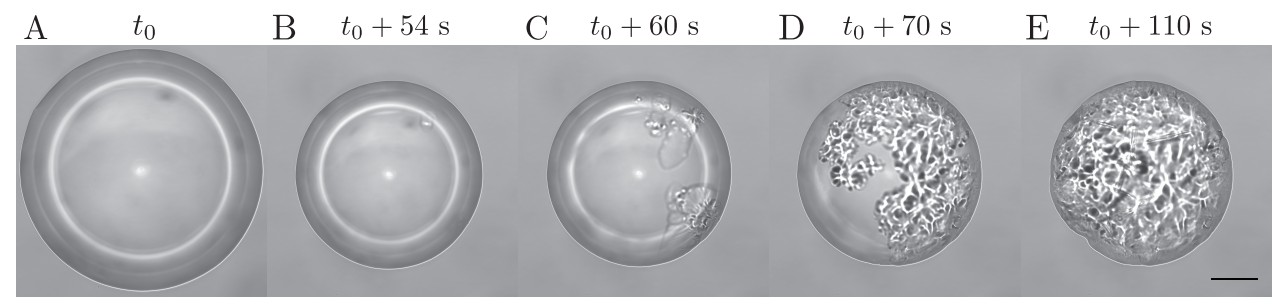

Figure 4.5: Bottom-view of a complete drop life time. (A) The drop evaporates on the substrate with receding contact line. (B) The first crystal appears near the contact line region. (C) Several crystals nucleate and grow independently. (D) Growing crystals coalesce with neighbouring ones. (E) The crystals cover the whole drop and bring the evaporation to an end. (B to E) The contact line basically remains the same until the final state of the drop, but slightly deforms due to the buckling of the drop surface. The scale bar represents $50 \mu \mathrm{m}$.
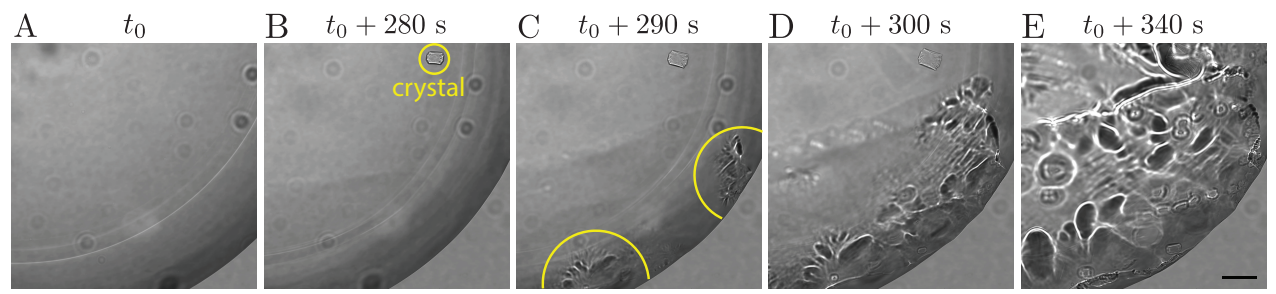

Figure 4.6: Bottom-view snapshots of the contact region of an evaporating surfactant-binary drop. (A) The moment of deposition of the drop: the drop starts evaporating on the substrate. (B) A small crystal nucleates (yellow circle), floats and grows near the contact line. (C) The crystals heterogenously nucleate at the contact line. (D) The nucleated crystals grow and merge with neighbouring crystals. (E) The crystalized SDS fully occupies the drop and eventually brings the evaporation to an end. The scale bar represents $20 \mu \mathrm{m}$.

trolled by the diffusion of the vapor away from the droplet $[6,59]$ : the liquid molecules change their phase and diffuse as vapor molecules into the surrounding air. Popov [5] derived an analytical solution by using the solution of the equivalent problem of an electric potential around a charged lens-shaped conductor:

$$
\frac{\mathrm{d} m}{\mathrm{~d} t}=-\pi D R\left(c_{\mathrm{s}}-c_{\infty}\right) f(\theta)
$$




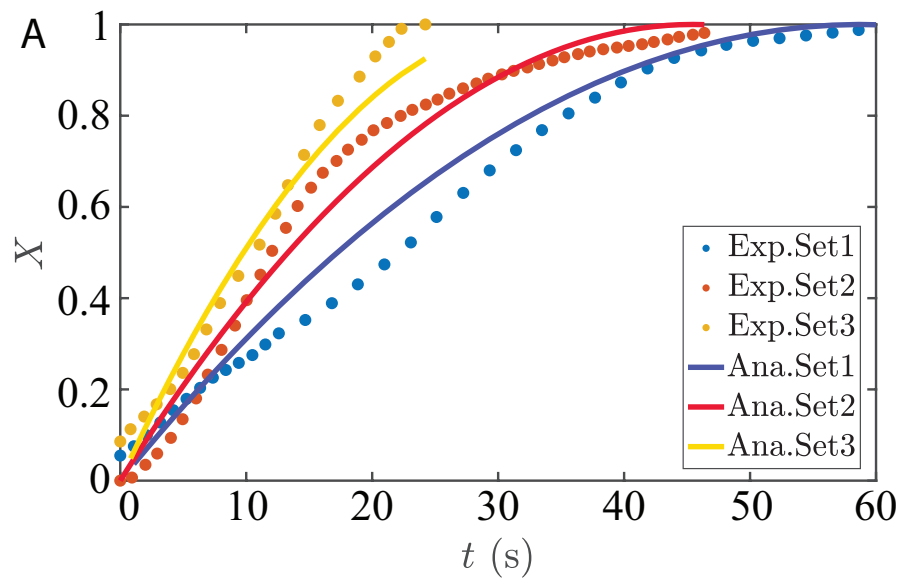

B

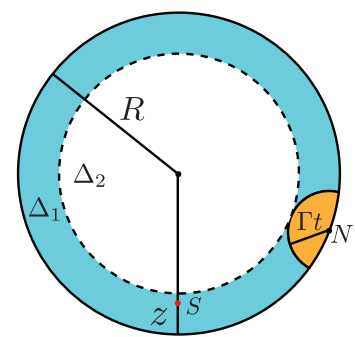

Figure 4.7: (A) Measurement of the crystallized area fraction of three droplets against time. The analytical results (solid lines) according to Eq. (4.7) are also shown. (B) The circular regions with areas $\Delta_{1}$ and $\Delta_{2}$, represent the areas where nucleation can or cannot occur in time $t$, respectively. The orange region indicates the area transformed at time $t$, due to a nucleation at N. $S$ is an arbitrary point in $\Delta_{1}$ which has distance $z$ from the boundary. Within time $t$, it must be transformed due to a nucleus on the boundary.

with

$$
f(\theta)=\frac{\sin (\theta)}{1+\cos (\theta)}+4 \int_{0}^{\infty} \frac{1+\cosh (2 \theta \varepsilon)}{\sinh (2 \pi \varepsilon)} \tanh [(\pi-\theta) \varepsilon] \mathrm{d} \varepsilon
$$

with $m$ the droplet mass, $D$ the diffusion coefficient of the droplet liquid vapor in air, $c_{s}$ the saturated concentration of liquid vapor molecules, and $c_{\infty}$ the ambient concentration of the liquid vapor far away from the drop.

For the evaporation of multicomponent droplets, we first employ the method suggested by Brenn [84], namely considering the total evaporation rate of the mixture droplet as the sum of the evaporation rate of each individual component. In our surfactant-laden glycerol-water droplet, glycerol and SDS are non-volatile under ambient condition [120]. Therefore, only the diffusive flux of water contributes to the total evaporation rate. The essential difference between the evaporation of pure droplets and multicomponent droplets is the vapor-liquid equilibrium: the non-volatile component in the system alters the saturated concentration of water vapor at the interface [24,45]. Raoult's law [121] is used to calculate the saturated water vapor concentration of the 
binary system: $c_{w, \mathrm{~s}}=X_{w} c_{w, \mathrm{~s}}^{0}$, where $X_{w}$ is the mole fraction of water at the interface and $c_{w, \mathrm{~s}}^{0}$ is the saturated vapor concentration of pure water. However, Raoult's law relies on an idealized solution and as such ignores any interaction between the components. To overcome this limitation, the so-called activity coefficient $\psi[122]$ was introduced to describe this interaction. In our case, it specifically addresses the interaction between water and the other components: $c_{\mathrm{w}, \mathrm{s}}=\psi_{\mathrm{w}} X_{\mathrm{w}} c_{\mathrm{w}, \mathrm{s}}^{0}$. By using the water activity coefficient $\psi_{\mathrm{w}}[10]$ in the modified Raoult's law, we obtain a theoretical model to express the evaporation rate for the binary droplet:

$$
\frac{\mathrm{d} m}{\mathrm{~d} t}=-\pi D R\left(\psi_{\mathrm{w}} X_{\mathrm{w}} c_{\mathrm{w}, \mathrm{s}}^{0}-c_{\mathrm{w}, \infty}\right) f(\theta) .
$$

There is, however, one added complexity in our system: it is difficult to determine the exact $c_{\mathrm{w}, \mathrm{s}}$ without knowing the exact mole fraction of water, glycerol, and SDS molecules. Hence we cannot analytically predict the evaporation rate for each time instant. To compare different sets of experimental data, we rescale the measured droplet volume and time, by introducing the nondimensional volume $\hat{V}=V / V_{0}$ and time $\hat{t}=t / \tau_{c}$, with $V(t)$ the measured droplet volume and $V_{0}$ its initial volume. $\tau_{c}$ is the characteristic timescale of the diffusive evaporation $[4,9]$, which can also be read from Eq. (4.1),

$$
\tau_{c}=\frac{\rho R_{0}^{2}}{D \Delta c} .
$$

Figures 4.3A2,B2,C2 show that the rescaled experimental data for volume $V / V_{0}$, contact angle $\theta$ and footprint radius $R / R_{0}$ versus the dimensionless time $t / \tau_{c}$ follow a universal curve for all measured droplet sizes. The collapse of all the curves demonstrates that regardless of the initial size, the droplets with the same initial composition always follow the same evaporation behavior, with a universal evolution of all geometrical characteristics. Based on this, we can conclude that the variations of not just the geometry but also of the internal composition concentration and distribution are universal, independent of the droplet size.

We also compare the initial evaporation rate of different initial volumes by introducing the dimensionless volume loss rate $\mathrm{d} \hat{V} / \mathrm{d} t=\mathrm{d}\left(V / V_{0}\right) / \mathrm{d} t$. According to Eq. (4.3), the dimensionless initial evaporation rate is

$$
\left.\frac{\mathrm{d} \hat{V}}{\mathrm{~d} t}\right|_{t=0} \propto \frac{D \Delta c R_{0}}{\rho V_{0}} \propto \frac{D \Delta c}{\rho V_{0}^{2 / 3}} .
$$


Based on the $V_{0}^{-2 / 3}$ proportionality of Eq. (4.5), we rescale the experimental data of Fig. 4.4A, see Fig. 4.4B, and plot them on a double logarithmic scale. Indeed, the data follow the scaling law as suggested by Eq. 4.5, confirming our model assumptions.

Besides controlling the evaporation rate, the model also yields the terminal state of the evaporation, which is when the saturated water vapor concentration equals the environmental concentration, $c_{w, s}=c_{w, \infty}$. Essentially, the evaporation stops when the active mole fraction of water equals the relative humidity $H$ of the surrounding air, $\psi_{w} X_{w}=H$. For the same reason as mentioned above, we only compare the experimental data with the analytical prediction for glycerol-water binary system, ignoring the mole fraction of the surfactant. From the relative humidity $H$ measured in experiment, we can calculate analytically the "theoretical final volume" $V_{t}$ (see Supplementary Materials) as

$$
V_{t}=\left(\frac{M_{w}}{M_{g}} \frac{H}{\psi_{w}-H}+\frac{\rho_{w}}{\rho_{g}}\right)\left(\frac{1-C_{g}}{C_{g}}+\frac{\rho_{w}}{\rho_{g}}\right)^{-1} V_{0},
$$

where $M_{g}=9.21 \times 10^{-2} \mathrm{~kg} / \mathrm{mol}$ and $M_{w}=1.8 \times 10^{-2} \mathrm{~kg} / \mathrm{mol}$ is the molecular mass of glycerol and water, respectively, $\rho_{g}=1.226 \times 10^{3} \mathrm{~kg} / \mathrm{m}^{3}$ and $\rho_{w}=$ $0.997 \times 10^{3} \mathrm{~kg} / \mathrm{m}^{3}$ are their liquid densities at room temperature, and $C_{g}$ is the initial mass concentration of glycerol in each measurement. The final volume of the equilibrium state (dashed line in Fig. 4.3A2) lies below the final volumes of all the droplets, which indicates that the shielding of water by the crystallized interface blocked any further evaporation before the system reached its equilibrium state.

\subsubsection{Theory of 2-dimensional finite system crystalization}

As it is well-known, the evaporation rate has a singularity at the rim of the droplet, provided the contact angle is smaller than $90^{\circ}[12,24]$, which in our system, where the contact angle ranges from $65^{\circ}$ to $40^{\circ}$ during the evaporation process, indeed is the case. The singularity implies that the water depletes the fastest at the rim, which locally leads to a higher concentration of glycerol at that part. It is therefore also expected that crystal nucleation occurs first near the CL region due to the highest degree of oversaturation of SDS.

To model the crystallization, we employ a 2-dimensional model which is derived by extending the JMAK formalism [123-127] to a finite 2-dimensional system with non-uniform nucleation. Based on the spherical shape of the 
droplet, the footprint area is circular and the nucleation starts near the contact line. We assume that the crystallization process occurs within a circular region, and nucleation is permitted at $t=0$ at various points on the perimeter of the area. Figure $4.9 \mathrm{~B}$ shows the geometry of the two regions $\Delta_{1}$ and $\Delta_{2}$ within a circle with radius $R$ (drop radius): the $\Delta_{2}$ region is completely free of crystallization, while $\Delta_{1}$ is partially crystalline. The maximum growth radius of the crystals is given by $\Gamma t$, where $\Gamma$ is the constant growth rate. Weinberg $[128,129]$ proposed an analytical model to describe the fraction $X(t)$ transformed at time $t$, namely

$$
X(t)=\left[1-(1-\Gamma t / R)^{2}\right] X_{1}(t)
$$

where $X_{1}(t)$ represents the fraction which has crystalized in $\Delta_{1}$. It can be expressed as [129]

$$
X_{1}(t)=1-\int_{1-y}^{1} \exp \left[-2 P_{1} R \cos ^{-1}\left(\frac{1+\Phi^{2}-y^{2}}{2 \Phi}\right)\right] \Phi \mathrm{d} \Phi \times \frac{1}{2}\left[1-(1-\Phi)^{2}\right]^{-1},
$$

with $\Phi=(R-z) / R$ and $y=\Gamma t / R . \quad z$ denotes the distance between an arbitrary point $S$ in the $\Delta_{1}$ region and the boundary. $P_{1}$ is the nucleation probability per unit length in region $\Delta_{1}$.

We demonstrate that the transformation rate is more sensitive to the growth rate $\Gamma$ rather than to the seeding probability $P_{1}$, as shown in Supplementary Materials. Here we set $P_{1}=1000 \mu \mathrm{m}^{-1}$ by assuming a saturated nuclei density. We test this theory for the three cases in Fig. 4.9A with droplet footprint radius $R_{1}=146 \mu \mathrm{m}, R_{2}=110 \mu \mathrm{m}$ and $R_{3}=86 \mu \mathrm{m}$. By fitting the theoretical curves to the experimental data, we obtain the growth rate as the fitting parameter: Quite consistently, the results are $\Gamma_{1}=2.48 \mu \mathrm{m} / \mathrm{s}, \Gamma_{2}=2.42 \mu \mathrm{m} / \mathrm{s}$, and $\Gamma_{3}=2.58 \mu \mathrm{m} / \mathrm{s}$ for the three analyzed cases. From Fig. $4.6 \mathrm{C}$, we estimate the crystal growth rate in the early crystallization stage by measuring the increasing rate of crystal size near the contact line within the yellow circle. We obtain the estimate $\Gamma \approx 25 \pm 5 \mu \mathrm{m} / 10 \mathrm{~s}=2.5 \pm 0.5 \mu \mathrm{m} / \mathrm{s}$, which is comparable to the values $\Gamma_{1}, \Gamma_{2}$, and $\Gamma_{3}$ obtained from our model. Even though we applied a $2 \mathrm{D}$ model to a $3 \mathrm{D}$ problem, the theoretical predictions show good agreement with experimental data: the reason is that our droplet is relatively flat, with a contact angle of about $40^{\circ}$ when crystallization occurs. 


\subsection{Conclusions and outlook}

In summary, crystallization of sodium dodecyl sulfate induced by selective evaporation in a surfactant-laden glycerol-water mixture droplet is observed during the evaporation process. We studied experimentally the dynamics of evaporation prior to the occurrence of crystal nucleation and the kinetics of crystallization, thereafter. We applied a diffusion model extended by Raoult's law to describe the evaporation characteristics and could reveal a universal evaporation behavior, independent of the size of the droplets. Finally, we applied a 2-dimensional model building on the JMAK nucleation model to describe the kinetics of the crystallization. Thanks to the the low contact angle, this model can successfully describe our experimental data on nucleation.

Surfactants attract significant attention as their ubiquitous role in fluid dynamics of either nature or technology [130]. Our findings clearly show an unexpected consequence of using surfactants in such evaporating systems. This particularly holds for inkjet printing where surfactants are extensively used. As nearly all inks contain various components with different volatilities, the variations of the composition ratio caused by the selective evaporation of more volatile components may lead to the segregation of surfactants in the form of liquid phase separation [64] or crystallization. Our study may rise the awareness of using surfactants with cautions in such multicomponent systems, which normally involves rich physicochemical processes [131].

Some issues remain open and unexplored. As the temperature can change the CMC of SDS in glycerol-water mixture [132], does the crystallization behavior also depend on the temperature? How to describe the buckling behavior after the occurrence of crystallization? Another question is on the morphology of the SDS crystals, e.g., is the crystal structure different from the one induced upon cooling? Such questions are of great interest in view of crystal chemistry, and it is worthwhile to further investigate such crystallization behavior from a microscopic perspective in the future.

\subsection{Supplemental Materials}

\subsubsection{Derivation of the theoretical final volume}

The molar fraction of water in a binary mixture is defined as the number of moles of water divided by the number of moles of both liquids. Here we neglect the mole fraction of SDS. Be $a$ the mass of water and $b$ the mass of another liquid, which is glycerol in our experiment. Then the mole fraction of water is 


$$
x_{\mathrm{w}}=\frac{a / M_{\mathrm{w}}}{a / M_{\mathrm{w}}+b / M_{\mathrm{g}}}=\frac{1}{1+\frac{b}{a} \frac{M_{\mathrm{w}}}{M_{\mathrm{g}}}} .
$$

Here we assume that the density of the mixture is linear with the solute concentration, which means that the total volume of the mixture is the sum of the individual liquid volume [108]. This is a reasonable approximation for water/glycerol mixtures. Then the initial density is given by

$$
\rho_{i}=\frac{m_{c}+m_{w}}{\frac{m_{c}}{\rho_{c}}+\frac{m_{w}}{\rho_{w}}}=\frac{\left(m_{c}+m_{w}\right) \rho_{c} \rho_{w}}{m_{c} \rho_{w}+m_{w} \rho_{c}},
$$

where $\rho_{i}$ is the initial density of the mixture, $m_{c}, \rho_{c}$ are the mass and density of the solute liquid, and $m_{w}, \rho_{w}$ are the mass and density of water. We introduce the initial mass percentage $c$ of the solute, whose value can be between 0 and 1. Then we have $m_{w}=m_{c} \frac{1-c}{c}$ at the beginning before evaporation. By substituting this into Eq. (4.10), it reduces to

$$
\rho_{i}=\frac{\left(m_{c}+m_{c} \frac{1-c}{c}\right) \rho_{c} \rho_{w}}{m_{c} \rho_{w}+m_{c} \frac{1-c}{c} \rho_{c}}=\frac{\rho_{c} \rho_{w}}{\rho_{c}+c\left(\rho_{w}-\rho_{c}\right)} .
$$

The solute mass in the droplet is now given by $V_{i} \rho_{i} c$, which is constant during the drying process due to the non-volatility of glycerol. $V_{i}$ is defined as initial droplet volume. Therefore the amount of water that has evaporated is given by $\left(V_{i}-V\right) \rho_{w}$, and the total mass of water left in the droplet is the initial mass minus the evaporated mass, $V_{i} \rho_{i}(1-c)-\left(V_{i}-V\right) \rho_{w}$. By substituting the water mass into Eq. (4.9), the mole fraction of water in the droplet can be expressed as

$$
x_{w}=\frac{1}{1+\frac{V_{i} \rho_{i} c}{V_{i} \rho_{i}(1-c)-\left(V_{i}-V\right) \rho_{w}} \frac{M_{w}}{M_{c}}} .
$$

Combing Eq. (4.12) and Eq. (4.11), it gives

$$
x_{w}=\frac{1}{1+\frac{1}{\frac{V}{V_{i}} \frac{1-c}{c}+\frac{V-V_{i}}{V_{i}} \frac{\rho_{w}}{\rho_{c}}} \frac{M_{w}}{M_{c}}} .
$$

From the theory, it is predicted that the droplet stops evaporating at the moment when the active mole fraction of water equals to the relative humidity $H$ of the surrounding air, $\psi_{w} x_{w}=H$. Then we obtain the theoretical final mole fraction $x_{w}=H / \psi_{w}$, where $H$ and $\psi_{w}$ are the relative humidity and 
the activity coefficient of water [10], respectively. By substituting $x_{w}$ into Eq. (4.13), we can analytically calculate the theoretical final volume $V_{t}$ :

$$
V_{t}=\left(\frac{M_{w}}{M_{c}} \frac{H}{\psi_{w}-H}+\frac{\rho_{w}}{\rho_{c}}\right)\left(\frac{1-c}{c}+\frac{\rho_{w}}{\rho_{g}}\right)^{-1} V_{0} .
$$

\subsubsection{Image analysis for crystallization process}

The crystallization process was observed using a Nikon eclipse (confocal) microscope, with a $60 \mathrm{x}$ magnification water objective focusing on the bottom layer of the droplet $(\approx 10 \mu \mathrm{m}$ above the substrate). A manual detection with ImageJ was used to measure the area which has already been crystallized at the certain moment. The boundary of the crystallization area is labeled by the yellow line, as shown in Fig. 4.8.

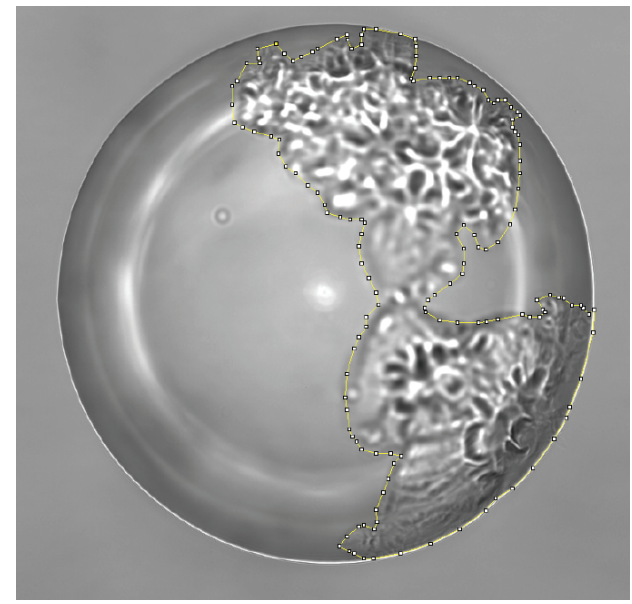

Figure 4.8: A snapshot of the image analysis by ImageJ. The crystallization area is manually detected and labeled by the yellow line. By counting the number of pixels within the area trapped within the yellow line, we measure the area of crystallization for every instant.

\subsubsection{Sensitivity of $P_{1}$ and $\Gamma$ in JMAK model}

We test the sensitivity of the seeding probability $P_{1}$ and growth rate $\Gamma$ in JMAK model. The time interval $\Delta t$ is set to be from 0 to $100 \mathrm{~s}$ and the radius of circular area $R$ is set to be $100 \mathrm{~m}$, which makes the characteristic growth 
rate $R / \Delta t=1 \mathrm{~m} / \mathrm{s}$. We vary $P_{1}$ from 0.01 to $1000 \mathrm{~m}^{-1}$ and $\Gamma$ from 0.01 to $32 \mathrm{~m} / \mathrm{s}$. The results are shown in Fig. 4.9, which clearly show that the JMAK model is sensitive to the change of $\Gamma$ (Fig. 4.9B) but insensitive to the choice of $P_{1}$ (Fig. $4.9 \mathrm{~A}$ ).
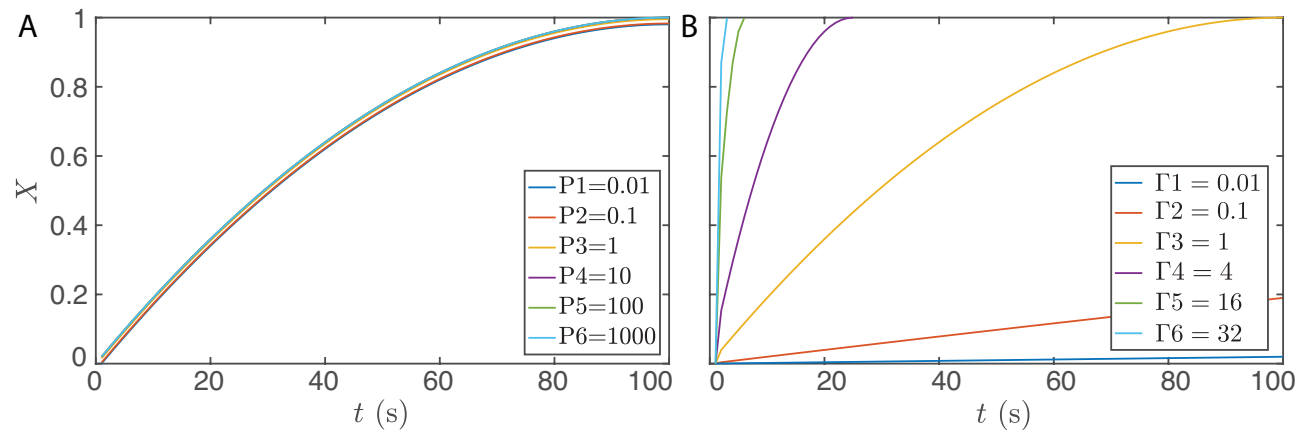

Figure 4.9: Calculated transformed area fraction with various seeding possibility $P_{1}$ and growth rate $\Gamma$ using JMAK model. 



\section{Chapter 5}

\section{Evaporating droplets on oil-wetted surfaces: Suppression of the coffee-stain effect*}

The evaporation of suspension droplets is the underlying mechanism in many surface coating and surface patterning applications. However, the uniformity of the final deposit suffers from the coffee-stain effect caused by contact line pinning. Here, we show that control over particle deposition can be achieved through droplet evaporation on oil-wetted surfaces. We demonstrate by flow visualization, theory, and numerics that the final deposit of the particles is governed by the coupling of the flow field in the evaporating droplet, the movement of its contact line, and the wetting state of the thin film surrounding the droplet. We show that the dynamics of the contact line can be tuned through the addition of a surfactant, thereby controlling the surface energies, which then leads to control over the final particle deposit. We also obtain an analytical expression for the radial velocity profile which reflects the hindering of the evaporation at the rim of the droplet by the non-volatile oil meniscus, prevents flow towards the contact line, thus suppressing the coffee-stain effect. Finally, we confirm our physical interpretation by numerical simulations that are in qualitative agreement with the

*Based on: Yaxing Li, Christian Diddens, Tim Segers, Herman Wijshoff, Michel Versluis, and Detlef Lohse, Evaporating droplets on oil-wetted surfaces: Suppression of the coffee-stain effect. Proc. Natl. Acad. Sci. U.S.A. (2020) (In press). Experiments are done by Li. Numerical Simulation is done by Diddens. 
experiment. 



\subsection{Introduction}

A coffee drop drying on a table top leaves a ring-like stain behind, a mechanism which is well-known as the "coffee-stain effect" [12]. It works as follows: When a coffee drop is evaporating with a pinned contact line, suspended particles are carried from the droplet interior towards its edge, and are left concentrated along the original drop edge. The reason lies in the fact that to maintain a pinned contact line, the evaporated liquid at the contact line needs to be replenished by liquid from the droplet's interior, thereby transporting particles to the contact line. The ubiquitousness of the coffee-stain effect makes it paradigmatic for scientific research, but for many practical applications it is a hinderance hard to overcome due to self-pinning caused by the suspended colloids [133]. However, in a wide variety of modern applications involving the evaporation of colloidal drops, such as in inkjet printing [25,134], surface patterning $[26,135]$ and 3D printing technologies [58], the uniformity of the final deposit is key. Therefore, ever since the coffee-stain effect has been revealed more than 20 years ago, extensive research has been dedicated, and is still dedicated, to control the phenomenon with the ultimate goal of its prevention in order to homogeneously deposit particles through droplet evaporation.

Within the studies that aim for control over particle deposition by droplet evaporation, the approaches mainly focus on internal flow manipulation [59, 136-139] and particle-solid interactions [140-143]. In particular, Marangoni effects in evaporative multicomponent systems, either solutal $[31,85,86,134$, $144,145]$ or thermal [146], have been suggested as an effective way to suppress outward capillary flow by continuously mixing the suspended particles during the evaporation process. However, recent experimental observations on the evaporation of multicomponent drops show complex physicochemical dynamics that can be triggered by selective evaporation such as phase separation $[24,47$, 64] and crystallization [147]. These systems are typically far from equilibrium, i.e., with gradients in concentration, which may bring uncertainties in the final deposition pattern. Notably, in very recent studies, the segregation in binary droplets even causes gravity-dominated flow [79, 88, 148], which may further complicate uniform particle deposition through droplet evaporation.

In a parallel line of research, lubricated surfaces have recently attracted a lot of attention due to their robust wetting properties for droplets deposited on their top $[8,101,149-154]$, as on such lubricated surfaces contact line pinning is reduced and the mobility of the droplets is strongly increased with respect to that of the non-coated surface. Although there are many studies on the 
mechanism of the extreme liquid repellency of lubricated surfaces [102,155], the drying behavior of a droplet on them has barely been touched upon, let alone fully understood. Only recently, Guan et al. [156] reported a study on the evaporation of sessile droplets on slippery liquid-infused porous surfaces, in which they mainly focused on the evaporation modes of droplets on such lubricated surfaces. Even more recently, Gao et al. [100] studied the flow behavior and particle deposition in evaporating colloidal droplets on oil-coated surfaces. Through varying oil-film parameters such as thickness and viscosity as well as the hydrophilicity of substrates, they achieved an improved control on supraparticle formation and on uniform particle deposition.

Here, we approach the problem in a different manner, that is, by controlling particle deposition through the surface energy of the droplet. We reveal the key characteristics of the evaporation of suspension droplets on oil-wetted surfaces through experiments, theory, and numerics by which we show that the oil-wetted surface allows for suppression of the coffee-stain effect and that thanks to the controllably either a "coffee-eye" [139] or a uniform particle coating can be achieved in the very same system, simply by tuning a single parameter: the surface energy of the droplet. The present work therefore opens up new application perspectives for surface coatings, particle patterning, and self-assembly. Furthermore, it is of great interest for industrial inkjet printing, specifically to control the final deposit of colloidal pigment particles that are typically destabilized by salts in a primer layer printed prior to ink deposition [101].

The essence of our physical model system is shown in Fig. 5.1(a1): a water droplet (MilliQ, Millipore Corporation) sits on an immiscible silicone oil film (Sigma-Aldrich, viscosity $\mu_{0} \approx 1 \mathrm{~Pa} \cdot \mathrm{s}$ ) with a thickness $h_{0}$ of $18 \pm 1 \mu \mathrm{m}$ that was spin-coated on a solid glass substrate (Gerhard Menzel GmbH, $76 \times 26$ $\mathrm{mm})$. The water droplet was then left to evaporate at ambient conditions. An oil meniscus ("wetting ridge"), rapidly forms around the deposited droplet to balance its three-phase contact line, as shown in Fig. 5.1(b1) with $\vec{\gamma}_{\text {oa }}+\vec{\gamma}_{\text {do }}+$ $\vec{\gamma}_{\mathrm{da}}=0$ [150], where $\vec{\gamma}$ is the directional interfacial tension, and the subscripts $\mathrm{a}, \mathrm{o}$, and $\mathrm{d}$ indicate the air and oil phases, and the droplet, respectively. When the spreading coefficient, $S_{\text {ow(a) }}=\gamma_{\text {wa }}-\gamma_{\text {wo }}-\gamma_{\text {oa }}$ [153] is larger than zero, the oil engulfs the droplet [Fig. 5.1(a1)] to minimize its surface energy. On the other hand, when $S_{\text {ow (a) }}<0$ the main part of the water droplet remains uncovered by the oil [Fig. 5.1(b1)]. Note that in the schematic in Fig. 5.1(a1, b1), the droplets are in direct contact with the substrate, meaning that no intercalated oil film between the droplet and the substrate is present. This was 
confirmed by interferometry measurements as detailed in the supplementary information.

a

pure water droplet

a1

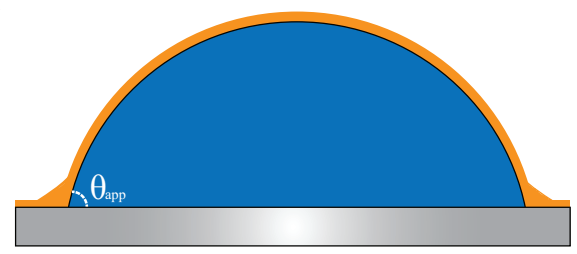

a2

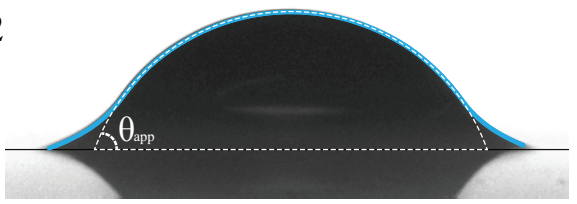

a3

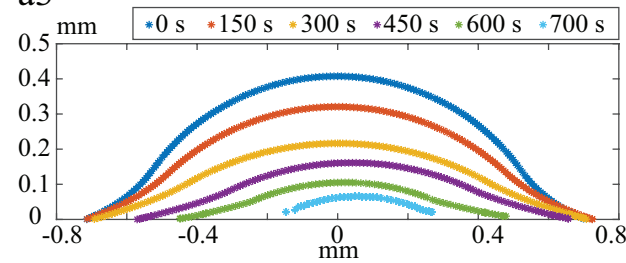

b surfactant-laden water droplet

b1

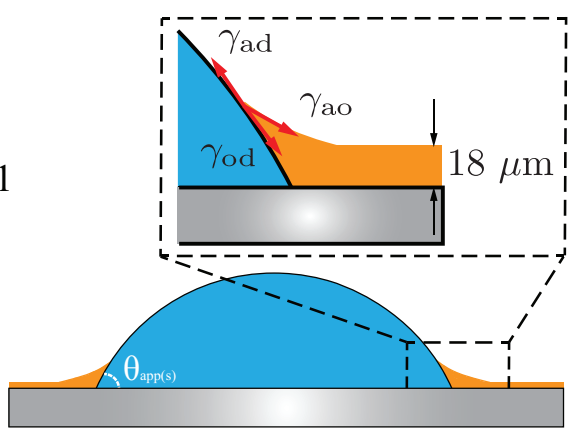

b2

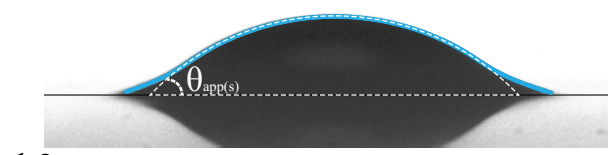

b3

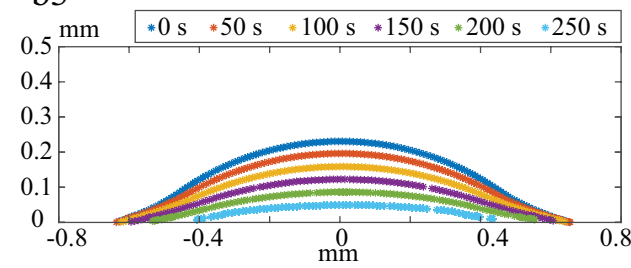

Figure 5.1: Schematic of a water droplet (a1) and a surfactant-laden water droplet (b1) sitting on an oil-wetted surface. The inset of b1 illustrates the surface tensions at the three-phase contact line and the thickness of the film $(18 \mu \mathrm{m})$. (a2, b2) Side view images of both droplets at the start of the evaporation process. (a3, b3) Temporal evolution of the side view profiles of both droplets. The length scales in a3, b3 are identical.

\subsection{Experimental results}

\subsubsection{Flow visualization}

As a reference, we start our experiments by quantifying the temporal evolution of both an evaporating oil-engulfed particle-laden $\left(2 \times 10^{-2}\right.$ vol\%, $0.52 \mu \mathrm{m}$ 
particle diameter, Fluoro-Max, Red Flourescent Polymer Microspheres) water droplet and that of a non-engulfed particle-laden water droplet. The engulfed droplet comprised water $\left(\gamma_{\text {oa }} \approx 21 \mathrm{mN} / \mathrm{m} ; \gamma_{\text {wo }} \approx 42 \mathrm{mN} / \mathrm{m} ; \gamma_{\text {aw }} \approx 72 \mathrm{mN} / \mathrm{m}\right)$ with a resulting spreading coefficient $S_{\text {ow(a) }}$ of $9 \mathrm{mN} / \mathrm{m}$. The non-engulfed state was reached by lowering the surface energies of the air-water and the

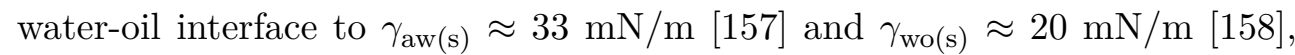
respectively, through the addition of a surfactant (Sodium Dodecyl Sulfate; SDS) at a concentration of 2 times the critical micelle concentration (1 CMC $\approx 2.36 \mathrm{~g} / \mathrm{L}[159])$. The resulting spreading coefficient is negative $(-8 \mathrm{mN} / \mathrm{m})$, corresponding to a non-engulfed state [Fig. 5.1(b1)]. The temporal evolution of the height profiles taken from a side-view recording of the evaporating droplets [Fig. 5.1(a2, b2)] are shown in Fig. 5.1(a3, b3). The apparent contact angle $\theta_{\text {app }}$ as defined by Guan et al. [156], can be measured from the profiles. We use the theory suggested by Semprebon et al. [154] to approximate the initial apparent contact angle for both cases in our study: $\cos \theta_{\text {app }}=\frac{\gamma_{\mathrm{oa}}-\gamma_{\mathrm{ds}}}{\gamma_{\mathrm{eff}}}$, where $\gamma_{\mathrm{ds}}$ is the interfacial tension between droplet and solid substrate, $\gamma_{\text {eff }}$ the effective interfacial tension, which is given by $\gamma_{\text {eff }}=\gamma_{\text {aw }}$ when $S_{\text {ow (a) }}<0$ or $\gamma_{\text {eff }}=\gamma_{\text {wo }}+\gamma_{\text {oa }}$ when $S_{\text {ow (a) }}>0$ [160]. Hence, for the first case (the water droplet on the oil-wetted surfaces, $\left.S_{\text {ow(a) }}>0\right): \cos \theta_{\text {app }}=\frac{\gamma_{\text {oa }}-\gamma_{\mathrm{ds}}}{\gamma_{\text {wo }}+\gamma_{\mathrm{oa}}} \approx 0.33$, where $\gamma_{\mathrm{ds}} \approx 0$ due to the almost complete wetting of water on the glass slide. Then $\theta_{\text {app }}=\arccos (0.33) \approx 70.7^{\circ}$. For the second case (the surfactant-laden water droplet on the oil-wetted surfaces, $\left.S_{\text {ow (a) }}<0\right): \cos \theta_{\text {app }(\mathrm{s})}=\frac{\gamma_{\mathrm{oa}}-\gamma_{\mathrm{ds}}}{\gamma_{\mathrm{aw}(\mathrm{s})}} \approx$ 0.64 . Then $\theta_{\mathrm{app}(\mathrm{s})}=\arccos (0.64) \approx 50.2^{\circ}$. From the experiment, we measure that $\theta_{\text {app }} \approx 70^{\circ}$, and $\theta_{\text {app }(\mathrm{s})} \approx 48^{\circ}$, which are close to the prediction by the theory $[154,160]$. Note that an annular "wetting ridge" was present for both droplets and that the engulfed water droplet (a2) had a much larger apparent contact angle than the non-engulfed droplet (b2). Also note that the engulfed droplet evaporated with a moving contact line whereas that of the non-engulfed droplet was pinned.

To further investigate the contact line behavior of both the edge of the droplet and that of the oil meniscus, bottom view thin-film interferometry experiments were performed using a confocal microscope. Therefore, monochromatic light with a wavelength of $561 \mathrm{~nm}$ was focused on the upper surface of the oil film and the reflected light was captured by a CMOS camera (see details in SI). The results for the evaporating droplets are shown in Figs. 5.2(a1-a3,b1b3). Here, the fringes are due to interference between the light reflected from the solid/oil and the oil/air interfaces whereas the dark ring results from the curved meniscus reflecting the light away from the aperture of the imaging 
lens. Note that the pure water droplet evaporated with a moving contact line [Figs. 5.2(a1-a2)] (Movie S4) and that after its evaporation, the suspended particles were left behind over a small area as compared to the initial droplet size. The fringes in Fig. 5.2(a3) show that the silicone oil film slowly filled the dimple left behind by the evaporated droplet. On the other hand, the surfactant-laden droplet evaporated with a pinned contact line [Fig. 5.2(b1b2)] (Movie S5). In the final stage of its lifetime, the ring-shaped oil meniscus touched on the substrate after the water underneath dried out (area between the red dotted ring and the yellow dotted ring) and it did not retract to the original contact line. The inner region of the substrate uncovered by the oil meniscus (yellow dotted ring) was directly exposed to the air with only particles left on the substrate. Thus the area over which particles are deposited is controlled by the contact line behavior during the evaporation process.

The observed contact line pinning of the surfactant-laden droplet results from surface energy minimization, i.e., the interfacial energy between the droplet and the substrate is lower than that between the oil and the substrate. Hence, the interfacial area remains unchanged to maintain the minimal surface energy of the system. When the initial SDS concentration was lower than 0.17 CMC $(0.4 \mathrm{~g} / \mathrm{L})$, a "half-pinned" regime was observed with both a free moving and a pinned contact line, as shown schematically in Fig. 5.2(c): the contact line first slides and then sticks due to the decreased surface energy resulting from the evaporation driven increase in SDS concentration. This suggests that the area over which particles are deposited can be controlled by the initial surfactant concentration. The evaporation state, i.e., half-pinned versus pinned, of surfactant-laden droplets is indicated by the blue and yellow regimes in Fig. 5.2(c), respectively. We quantify the degree of contact line pinning by calculating the ratio of the final particle deposit area $A_{\mathrm{f}}$ and the initial contact area $A_{\mathrm{o}}$. In the "half-pinned" regime, it shows that the increasing initial concentration of SDS enlarges the ratio $A_{\mathrm{f}} / A_{\mathrm{o}}$. It clearly indicates that by tuning the surface energy of the droplet, the contact line behavior of the droplet on an oil-wetted surface can be controlled which in turn manipulates the size of final particle deposit.

We will now further demonstrate that the coupling of the motion of the oil meniscus and that of the contact line determines the flow field inside the droplet and thereby the final deposition pattern of the suspended colloidal particles. The flow field and the final particle deposit were quantified by microparticle image velocimetry ( $\mu$ PIV, see experimental details in supplementary information). The flow field was measured at the plane $2.0 \pm 0.5 \mu \mathrm{m}$ above the 
substrate. The corresponding fluorescence images for the pure water droplet showing bright particles against a dark background are shown in Fig. 5.3(a) at different time instants. The images were processed to obtain the time-resolved velocity fields, see Fig. 5.3(b). The mean radial velocity of the pure water droplet is shown in Fig. 5.3(c) as a function of time (positive values indicate outward flow). In the early stage, $t<620 \mathrm{~s}$, indicated by the vertical dotted line, the droplet evaporated in the "constant radius mode" with a continuously decreasing contact angle and with a barely detectable and irregular flow. The typical mean flow speed was $\bar{U} \approx 1 \mu \mathrm{m} / \mathrm{s}$, so that the Reynolds number $\operatorname{Re}$ $=\bar{U} h_{0} / \nu \approx 10^{-3}$, with the kinematic viscosity $\nu \approx 10^{-6} \mathrm{~m}^{2} / \mathrm{s}$. After $620 \mathrm{~s}$, the contact line started to recede, which led to an increase of the mean flow velocity with a flow speed at $\bar{U} \approx 2 \mu \mathrm{m} / \mathrm{s}$. When the droplet reached the end of its lifetime, the flow slowed down and a "coffee-eye" deposit was left behind on the substrate.

When the surfactant was added to the evaporating droplet at a concentration of 2 CMC $(4.73 \mathrm{~g} / \mathrm{L})$, the flow field changed dramatically. Figure 5.3(d) shows the fluorescence images from which the time-resolved velocity field was determined, see Fig. 5.3(e). In Fig 5.3(f), the local radial velocity $U_{\mathrm{r}}$ at the distance $R-r=80 \mu \mathrm{m}$ from the contact line and the mean radial velocity $\bar{U}_{\mathrm{r}}$ of the entire flow field are shown. During the first phase of the evaporation process $(t \lesssim 130 \mathrm{~s})$, the local gradient in surfactant concentration resulted in a Marangoni-driven flow, with a larger magnitude $(\bar{U} \approx 4 \mu \mathrm{m} / \mathrm{s})$ than the flow in the evaporating pure water droplet $(\bar{U} \approx 1 \mu \mathrm{m} / \mathrm{s})$. Note that the flow was still chaotic rather than radially towards the edge of the droplet, which suppresses the coffee-stain effect. At a later stage, radially outward streaming patterns appeared but instead of flowing all the way to the edge of the droplet, the outward flow stopped at the location indicated by the red dotted lines in Fig. $5.3(\mathrm{~d})$. Before $\mathrm{t}=271 \mathrm{~s}, \bar{U}_{\mathrm{r}}$ was always negative showing inward flow from the edge towards the interior of the droplet. After $t=271 \mathrm{~s}$, towards the end of the droplet's lifetime when its profile became very flat, flow-reversal occured and the flow velocity dramatically increased, a phenomenon known as "rush-hour" [21]. Nevertheless, in spite of the strong radially outward flow in the final stage of the evaporation process, the particle deposition pattern (Fig. 5.3(d) at $\mathrm{t}=304 \mathrm{~s}$ ) is nearly homogeneous.

\subsubsection{Quantification of final deposit}

We now quantify the final particle deposits for the three different cases presented in Fig. 5.4: (a) a colloidal water droplet on a glass slide, (b) a colloidal 


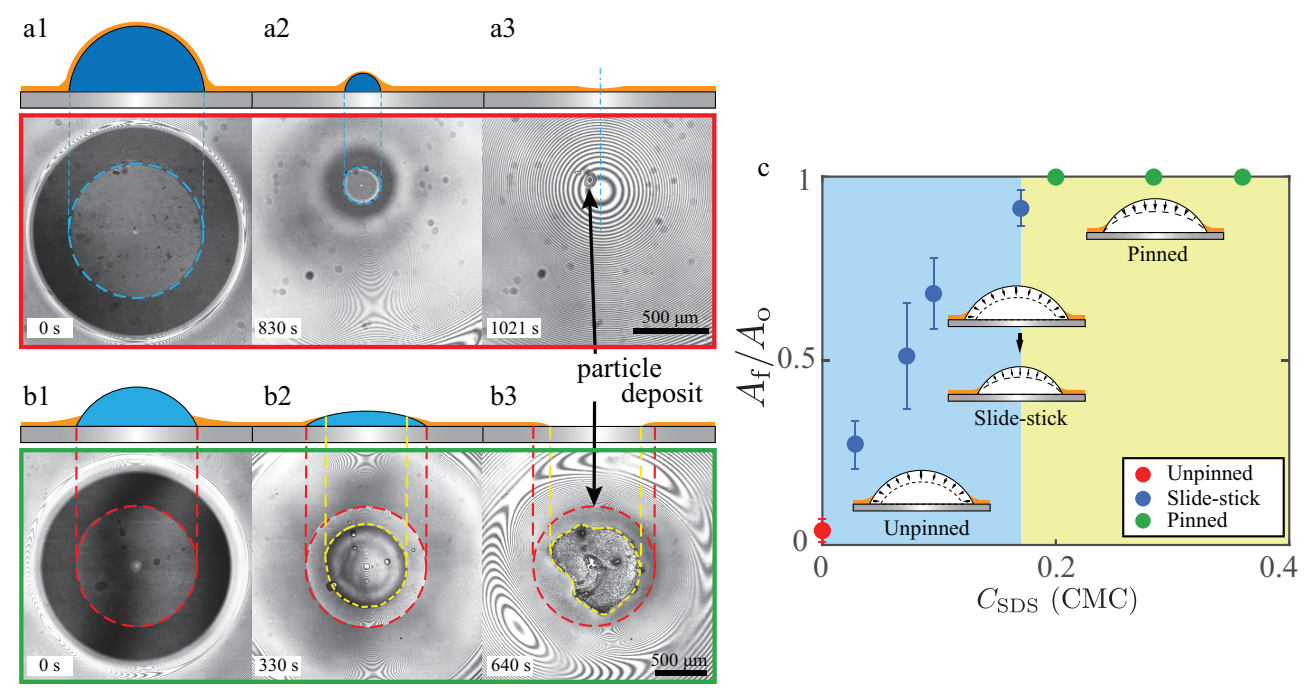

Figure 5.2: (a1-a3) Side view sketch and bottom view interferometric image of a water droplet evaporated on the oil-wetted substrate with a moving contact line. (a3) After evaporation a dimple in the silicone oil film is left behind that slowly fills up due to capillary action. (b1-b3) The same for an evaporating surfactant-laden water droplet $(2 \mathrm{CMC})$ on the substate with a pinned contact line. (b3) After the droplet fully evaporated, the oil meniscus fell down to the substate without retracting. By adding particles, we observed that in the interior (within yellow dashed line), the substrate was exposed to the air with only particles covering it, but within the region between the yellow and the red lines, the particles were entrapped by the oil meniscus. (c) The phase diagram displaying the transition from "unpinned" to "pinned" against SDS concentration in the droplet. $A_{\mathrm{f}} / A_{\mathrm{o}}$ represents the area of final particle deposit as compared to the initial contact area.

water droplet on an oil-wetted surface, and (c) a colloidal surfactant-laden water droplet on an oil-wetted surface. The initial particle distribution at the beginning of the evaporation process was homogeneous as can be observed from Fig. 5.4(a2) to (c2). However, the final deposition patterns after the droplets had fully evaporated were very different, see Figs. 5.4(a3) to (c3). The water droplet in absence of an oil film left behind a cascading-coffee-ring structure on the glass slide due to contact line pinning caused by heterogeneous surface roughness and contact line interaction with particles. On the other hand, the lubricant film dramatically changed the contact line mobility 

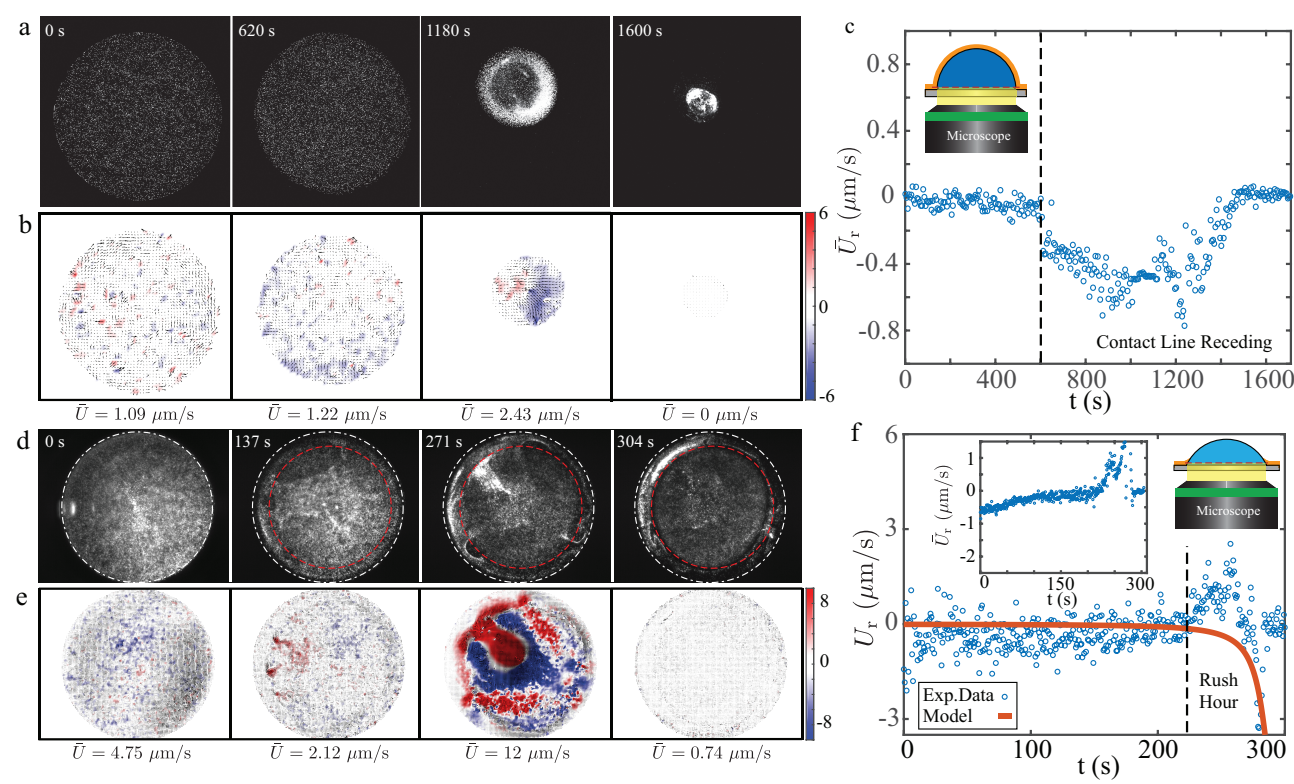

Figure 5.3: (a,d) Experimental snapshots of the particle distribution in the focal plane $\approx 2 \mu \mathrm{m}$ above the substrate at different time instants for a colloidal water droplet (a) and a surfactant-laden (2 CMC) colloidal water droplet (d), respectively, both evaporating on the oil-wetted surface. The red dashed circle in (d) indicates the inner boundary of the ring area, which is the regime covered by the wetting ridge. (b,e) Snapshots of the velocity field for each of the image in $(\mathrm{a}, \mathrm{d})$. (c) The evolution of the mean radial velocity $\bar{U}_{\mathrm{r}}$ for the water droplet in $(\mathrm{a}, \mathrm{b})$ : the vertical dashed line marks where the contact line starts to move. (f) The evolution of the local radial velocity $U_{\mathrm{r}}$ at the distance $R-r=80 \mu \mathrm{m}$ from the contact line for the surfactant-laden droplet in $(\mathrm{d}, \mathrm{e})$ : the dashed line indicates the beginning of the "rush hour" behavior: the radial velocity diverges towards the end of the droplet lifetime. The red solid line is our model Eq. 5.3, which well describes the final rush hour. The inset displays the mean area averaged radial velocity $\bar{U}_{\mathrm{r}}$ of the entire flow field.

such that during evaporation, the contact line moved inwards, thereby taking the particles along towards the droplet center, a phenomenon which is often called "self-lubrication" $[161,162]$. Note that the droplet was in direct contact with the glass substrate such that the oil film was forced to slide inwards with the contact line. For the surfactant-laden droplet, the contact line was pinned for most of the droplet's lifetime, thereby forming a nearly uniform deposition 
pattern. Indeed, for the final deposits, starting at their center, the fluorescent intensity was averaged over all angles to obtain the particle number density, and it was nearly uniform for the surfactant-laden droplet on the oil-wetted substrate, which can be appreciated from Figs. 5.4(a4) to (c4).

\subsection{Hydrodynamic model}

We now theoretically analyze the observed suppressed coffee-stain effect for the surfactant-laden droplet evaporating on an oil-wetted surface. The mechanism that suppresses radial flow towards the edge can be interpreted from massbalance considerations [21]. In contrast to the conventional coffee-stain effect, evaporation at the droplet edge is hindered by the nonvolatile oil meniscus. Hence, the evaporative flux diverges at the oil-water-air three-phase contact line, instead of the edge of the droplet. In the limit of small contact angles, the flux profile is given by $[18,107]$ :

$$
J(r, t)= \begin{cases}j_{0}\left[1-\left(r / R_{\text {in }}\right)^{2}\right]^{-1 / 2+\theta(t) / \pi} & \left(r<R_{\text {in }}\right), \\ 0 & \left(r \geq R_{\text {in }}\right),\end{cases}
$$

with $j_{0}=\frac{2}{\pi} \frac{D \Delta c}{R_{\text {in }}}, D=24 \times 10^{-6} \mathrm{~m}^{2} / \mathrm{s}$ the diffusion coefficient for vapor in air, $\Delta c=1.2 \times 10^{-2} \mathrm{~kg} / \mathrm{m}^{3}$ the vapor concentration difference between the drop surface and the surroundings and $R_{\text {in }}$ the radius of the area uncovered by the meniscus (the area within the red circle in Fig. 5.3(d)). The temporal dependence of the height-averaged radial velocity $\bar{u}(r, t)$ at a distance $r$ from the drop center is expressed as (see supplementary information):

$\bar{u}(r, t)= \begin{cases}-\frac{j_{0}}{\rho} \frac{\pi R_{\text {in }}^{2}}{(\pi+2 \theta) R^{3}} \frac{2\left(R^{2}-r^{2}\right)}{\theta r}+\frac{j_{0}}{\rho} \frac{\pi R_{\mathrm{in}}^{2}}{\pi+2 \theta} \frac{2 R}{\theta\left(R^{2}-r^{2}\right) r}\left(1-\frac{r^{2}}{R_{\mathrm{in}}^{2}}\right)^{1 / 2+\theta / \pi} & \left(r<R_{\mathrm{in}}\right), \\ -\frac{j_{0}}{\rho} \frac{\pi R_{\mathrm{in}}^{2}}{(\pi+2 \theta) R^{3}} \frac{2\left(R^{2}-r^{2}\right)}{\theta r} & \left(r \geq R_{\mathrm{in}}\right) .\end{cases}$

For $r \geq R$, the velocity $\bar{u}(r) \leq 0$, which indicates that the liquid flows from the edge towards the center within the contact line regime, hence avoiding the coffee-stain effect. In order to compare this simple model with the $\mu \mathrm{PIV}$ measurements, we note that those are performed at a focal plane $2.0 \mu \mathrm{m} \pm$ $0.5 \mu \mathrm{m}$ above the glass slide, where the measured velocity differs from the height-averaged velocity. Therefore we must also calculate the local radial velocity $U_{\mathrm{r}}(r, z, t)$ at distance $z=2 \mu \mathrm{m}$ from the substrate, which within the lubrication approximation of ref. [21] is given as 


$$
U_{\mathrm{r}}(r, z, t)= \begin{cases}\frac{3}{h^{2}(r, t)} \bar{u}(r, t)\left(h(r, t) z-\frac{1}{2} z^{2}\right) & \left(r<R_{\text {in }}\right), \\ \frac{6}{h^{2}(r, t)} \bar{u}(r, t)\left(h(r, t) z-z^{2}\right) & \left(r \geq R_{\text {in }}\right),\end{cases}
$$

with the local height of the droplet $h(r, t)=\theta(t)\left(R^{2}-r^{2}\right) /(2 R)$. In Fig. 5.3(f), we quantitatively compare the model with the measured radial velocity at distance $R-r=80 \mu \mathrm{m}$ from the contact line (within the regime covered by the wetting ridge, $r>R_{\text {in }}$ ). For the plot of the radial velocity predicted by Eq. 5.3 we took $z=2 \mu \mathrm{m}, R_{\text {in }}=380 \mu \mathrm{m}$ and $R=475 \mu \mathrm{m}$. The contact angle $\theta(t)$ is calculated based on the droplet's lifetime $t_{e}$, which is determined from experiment, see Supplementary Material. The model shows quantitative agreement with the experimental data, except for the small period $(t=230 \mathrm{~s}-$ $265 \mathrm{~s}$ ) in the early stage of the rush-hour behavior. The strong outward flow at the radial position close to $r=R_{\text {in }}$ explains the increase of the radial velocity in the early stage of the "rush-hour". In the experiment, the flow-reversal does not always perfectly occur at $r=R_{\text {in }}$. This is also due to the unpinning of the contact line. When the droplet becomes very flat due to the evaporation, the contact line detaches from one side and the contact area shrinks towards the other side. Because of this, the liquid tends to flow towards the pinning side, as shown in the third column of Fig. 5.3(d). This mechanism is also the reason for the uneven distribution of the particles in the very late stage of the droplet's lifetime.

\subsection{Numerical investigation}

To substantiate our interpretation regarding the suppression of the coffeestain effect in the surfactant-laden droplet on the oil-wetted substrate, we took advantage of numerical simulations. In Fig. 5.5(a), a snapshot of the simulation of a pure water droplet on a glass substrate with an advancing contact angle of $\theta_{\mathrm{a}}=20^{\circ}$ and a receding contact angle of $\theta_{\mathrm{r}}=10^{\circ}$ is shown. The slip velocity can be controlled by the choice of the slip length, which has been set to $l_{\mathrm{s}}=5 \times 10^{-14} \mathrm{~m}$ here to match approximately the slip duration in the experiments. As expected, the coffee-stain effect transports the particles towards the rim during the pinned phases in the evolution, whereas the contact lines moves rather quickly in the short intermediate slip regimes. The resulting deposition pattern, as shown in Fig. 5.5(c), reproduces the features of the experimentally obtained pattern (cf. Fig. 5.4(c1)), namely sharp concentric deposition rings stemming from the pinned evaporation phases. 
The very thin engulfing oil layer on the pure water droplet on the oil-wetted substrate, as depicted in Fig. 5.4(d2), is difficult to incorporate into a simulation. Moreover, the exact evaporation rate through this thin oil layer has not yet been quantified. To that end, we only present a simulation of the third case, i.e. the surfactant-laden water droplet on an oil-wetted substrate (cf. Fig. 5.4(d3)). In that simulation, instead of considering the presence of the surfactants directly, the surface tension of the droplet-gas interface has been artificially reduced to $\gamma_{\mathrm{ad}}=33 \mathrm{mN} / \mathrm{m}$. A snapshot of the simulation is shown in Fig. 5.5(b). It is clearly visible that there is still a singularity of the evaporation rate at the rim of the water-air interface. However, since it is not in contact with the substrate but with the oil meniscus, the enhanced particles at this position are not transported towards the rim of the droplet, i.e. to the pinned contact line between water, oil and the substrate. Instead, as already predicted in the analytical considerations (Eq. 5.2), the flow in the part of the droplet covered by the oil is directed inwards. This indeed leads to a rather homogeneous deposition pattern despite of the pinned contact line throughout the entire drying process, as shown in Fig. 5.5(d).

\subsection{Conclusions}

In summary, we have systematically studied particle-laden droplet evaporation on oil-wetted surfaces by experiment, theory and numerics. It is shown that the interfacial energies between the droplet, the oil film, and the solid substrate determine the wetting state, and moreover, influence the flow structure. We have demonstrated that by tuning the surface energy of droplets through the addition of surfactants, the contact line dynamics can be controlled such that either the coffee-stain effect can be suppressed to obtain a uniform deposition, or a size-controlled "coffee-eye" pattern can be formed. Numerically, we simulate the evaporation of a water droplet on a glass substrate and that of a surfactant-laden droplet evaporating with a meniscus arising from the oil film, showing a cascading coffee ring pattern and a uniform deposition, respectively. The results are in qualitative agreement with the experimental observations. This work therefore offers a useful and robust method to accomplish controlled particle deposition, which may open further application perspectives for surface coating, particle patterning and self-assembly. Moreover, our study focuses on the case that oil is coated on a hydrophilic substrate. It is definitely worthwhile to further explore the particle deposition modes on other lubricated surfaces such as LIS or SLIPS, which have more 
complicated surface features. The knowledge on particle deposition from evaporating particle-laden droplets on oil-wetted surfaces is also very relevant for disease transmission or in the agricultural context, where droplets are sprayed on leaves.

\subsection{Experimental Methods}

The evaporation process was recorded simultaneously from the side and the bottom (see Fig.1 in SI): the side view was used to characterize the geometrical parameters and the bottom view was used to implement the $\mu \mathrm{PIV}$ measurement. For the side view, the droplet was illuminated by a LED light source [MWWHL4 Warm White Mounted LED, THORLABS] from one side and recorded from the other side by a microscope [12X Ultrazoom, NAVITAR] connected to a CMOS camera [MQ013MG-E2, XiQ]. For the bottom view, a laser was used to excite the fluorescent particles [Fluoro-Max; Red Flourescent Polymer Microspheres: Ex/Em $530 \mathrm{~nm} / 607 \mathrm{~nm}$; Diameter: $0.52 \mu \mathrm{m}$ ] and a camera connected to a micro-lens was used to capture the fluorescent signal emitted by the particles. The two cameras were synchronized by a BNC pulse/delay generator. The typical frame rate for the measurement was 10 fps. The relative humidity in the laboratory was measured with a standard hygrometer $\left( \pm 3 \% \mathrm{RH}\right.$ for $35 \% \sim 70 \% \mathrm{RH}$ at $\left.20{ }^{\circ} \mathrm{C}\right)$. The temperature of each experiment was between $21{ }^{\circ} \mathrm{C}$ and $22{ }^{\circ} \mathrm{C}$. The relative humidity was around $40 \%$.

The silicone oil films were spincoated resulting in uniform thickness over the entire substrates. We controlled the film thickness by the spinning time and the rotation speed. The spinning time and rotation speed were fixed at $100 \mathrm{~s}$ and $25 \mathrm{rps}$. The thickness of the film was measured by a spectrometer (OceanOptics HR2000+ with HL-2000-FHSA halogen light source) to be 18 $\pm 1 \mu \mathrm{m}[163]$.

\subsection{Numerical Model}

A sharp-interface finite element method combined with an arbitrary LagrangianEulerian approach has been developed and implemented via the finite element library OOMPH-LIB [164]. Within this method, the droplet is considered to be axisymmetric. In the gas phase, the diffusion equation of water vapor is solved, by that the local evaporation rate at the interface is obtained. In the liquid, the Navier-Stokes equations determines the flow. The presence of 
multiple interfaces, even with a three-phase contact line between the three free interfaces (water-gas, oil-gas, water-oil), can easily be treated with this approach, as the contributions of the individual surface tensions to the stress are directly calculated at the sharp interfaces. The numerical method, which generalizes the method described in [46] by the possibility of having arbitrarily shaped interfaces, has been successfully used to reproduce experimental measurements on droplet evaporation [88], submerged droplets with competing buoyancy and Marangoni effects [165] and Leidenfrost droplets [166]. Since here we are also interested in deposition patterns predicted by the numerics, a convection-diffusion equation for the mass fraction of particles has been included and the most simple deposition model has been used, namely a deposition rate proportional to the mass fraction of particles in the liquid. Effects of particles on the fluid and on the contact line dynamics, as well as particleparticle interactions and thermal effects have been disregarded for simplicity. The deposition profile is given as an effective height based on the assumption of completely densely packed particles.

\subsection{Supplemental Materials}

\subsubsection{Experimental setup}

The evaporation process was recorded simultaneously from the side and the bottom (see Fig. 5.6): the side view was used to characterize the geometrical parameters and the bottom view was used to implement the $\mu \mathrm{PIV}$ measurement.

For the volumetric measurement from the side view, the image analysis was performed by custom-made MATLAB codes to detect the profile of the droplet and the wetting ridge. For the $\mu \mathrm{PIV}$ images, the noises in the background outside the droplet contour are first removed and exported as a new series of images for the processing. Then the post-possessing images were analyzed with PIVlab [51,52], utilizing an interrogation window of $32 \times 32$ pixels, followed by an analysis with sub-window of $16 \times 16$ pixels and then a second sub-window of $8 \times 8$ pixels.

\subsubsection{Intercalated film between a droplet and a flat solid sub- strate}

Previous studies have shown complex equilibrium lubrication states of the intercalated film sandwiched between a droplet and a flat solid surfaces, de- 
pending on the relation between the interfacial energies of droplet, liquid film, and substrate $[102,155]$. Three possible states were identified: 1 . a stable lubricant film beneath the droplet; 2 . an unstable film film beneath that forms discrete liquid pockets; and 3. a lubricant film that is completely displaced from beneath the droplet. To confirm that no intercalated lubricant film neither liquid pockets are present below our droplets, we performed thin-film interferometry [102]. Therefore, a monochromatic light with a wavelength $\lambda$ of $561 \mathrm{~nm}$ was focused on the upper surface of the substrate and the reflected light was captured by a confocal microscope. In the presence of an intercalated lubricant film, the light reflected off the solid/lubricant and lubricant/droplet interfaces interferes constructively or destructively to result in bright or dark fringes, respectively. Carlson et al. studied the stability of the oil-film under the water drop as a function of the water drop equilibrium contact angle $\left(\theta_{\mathrm{sw}}\right)$ on a dry substrate [151]. They found that for $\theta_{\mathrm{sw}}<90^{\circ}$ the oil film ruptures and water re-wets the substrate whereas for $\theta_{\mathrm{sw}}>90^{\circ}$ a stable film is formed. The glass substrate in our experiment has a $\theta_{\mathrm{sw}}$ of $10^{\circ}$ for the water drop and a $\theta_{\mathrm{sw}}$ of $8^{\circ}$ for the surfactant-laden drop. The absence of fringes in Figs. 5.7 (a,c) indeed demonstrates that both droplets fully touched the hydrophilic surface [167]. For a comparison, the interferometry measurements were repeated for the same droplet compositions and the same lubricant film but now deposited on hydrophobic OTS-coated glass with corresponding contact angle $\theta_{\mathrm{sw}} \approx 100^{\circ}$ for a pure water droplet and $\theta_{\mathrm{sw}} \approx 60^{\circ}$ for the surfactant-laden droplet. The presence of the fringes during the entire lifetime of the water drop, demonstrates that the silicone oil beneath the droplet was stable. For the surfactant-laden water drop, the rupture of the thin film evolved slowly and formed discrete liquid pockets. In this study, we focus on the hydrophilic substrate on which our water droplets are in direct contact with the substrate [Fig. 5.7(b,c)].

\subsubsection{Contact line behavior of oil meniscus}

We employed the interferometry but raised the focal plane to the upper surface of oil film. Fig 5.7(a2) schematically shows the measurement: the fringes are due to interference between the light reflected from the solid/oil and the oil/air interfaces. With the method, we are able to visualize the contact line motion of oil meniscus and the particle depositions on the substrate. 


\subsubsection{Hydrodynamic model}

The flow inside the droplet is driven by the evaporative flux from the droplet surface. We consider an axisymmetric droplet with a pinned contact line, which is described in a cylindrical coordinate system $(r, z)$; see Fig 5.8 [21]. Inside the droplet, we define an infinitesimally small control volume of width $\mathrm{d} r$, located at a distance arbitrary $r(r \leq R)$ from the center of the droplet. Mass conservation requires that the change rate of the amount of liquid inside the control volume equals to the net inflow of the liquid into the control, minus the depletion of the liquid due to the evaporation from the droplet surface of that volume. It can be expressed as

$$
\frac{\partial h}{\partial t}=\frac{1}{r} \frac{\partial}{\partial r} Q-\frac{1}{\rho} J
$$

where $h(r, t)$ is the local height of the liquid surface, $t$ is the time, $r$ is the radial coordinate, $Q(r, t)$ is the volume flow, $\rho$ is the liquid density, and $J(r, t)$ is the local evaporative flux. For small contact angle $\theta$ and small capillary number and Bond number, the local height can also be expressed with the geometrical relation:

$$
h(r, t)=\frac{R^{2}-r^{2}}{2 R} \theta(t) .
$$

In our system, the edge of the droplet is covered by a nonvolatile liquid, i.e., 1,2-hexanediol, which hinders the depletion of water. We assume that the evaporative flux of the area covered by the meniscus is zero and that of the inner part exposing to the air is obtained from the solution of the diffusion equation for vapor in air $[18,107]$ :

$$
J(r)= \begin{cases}j_{0}\left[1-\left(r / R_{\mathrm{in}}\right)^{2}\right]^{-1 / 2+\theta / \pi} & \left(r<R_{\text {in }}\right), \\ 0 & \left(r \geq R_{\text {in }}\right),\end{cases}
$$

where $j_{0}=\frac{2}{\pi} \frac{D \Delta c}{R_{\mathrm{in}}}$, with $D$ the diffusion coefficient for vapor in the air and $\Delta c$ the vapor concentration difference between the droplet surface and the surrounding air in the experiment. The change in the droplet volume is expressed as:

$$
\frac{d V}{d t}=\frac{d}{d t} \int_{0}^{R} h(r, t) 2 \pi r d r=\frac{\pi R^{3}}{4} \frac{d \theta}{d t},
$$

which should be equal to the total amount of evaporated liquid: 


$$
\frac{d V}{d t}=-\frac{1}{\rho} \int_{0}^{R} J(r) 2 \pi r d r=-\frac{2 \pi}{\pi+2 \theta} \frac{\pi j_{0} R_{\mathrm{in}}^{2}}{\rho} .
$$

By combing Eq. 5.7 and Eq. 5.8, we obtain:

$$
\frac{d \theta}{d t}=-\frac{8 \pi}{\pi+2 \theta} \frac{R_{\mathrm{in}}^{2}}{R^{3}} \frac{j_{0}}{\rho} .
$$

Hence, we obtain the temporal evolution of contact angle $\theta(t)$,

$$
\theta=\frac{1}{2} \sqrt{\frac{64 D \Delta c}{\rho} \frac{R_{\mathrm{in}}}{R^{3}}\left(t_{e}-t\right)+\pi^{2}}-\frac{\pi}{2},
$$

with $t_{e}$ the total droplet's lifetime. We substitute Eq. (5.9) into Eq. (5.4), then

$$
\frac{\partial h}{\partial t}=\frac{R^{2}-r^{2}}{2 R} \frac{\partial \theta}{\partial t}=-\frac{4 \pi\left(R^{2}-r^{2}\right)}{\pi+2 \theta} \frac{R_{\mathrm{in}}^{2}}{R^{4}} \frac{j_{0}}{\rho} .
$$

Then by replace $d h / d t$ in Eq. (5.6), we have

$$
\begin{aligned}
& \frac{1}{r} \frac{\partial}{\partial r} Q=-\frac{\partial h}{\partial t}-\frac{1}{\rho} J= \begin{cases}\frac{4 \pi\left(R^{2}-r^{2}\right)}{\pi+2 \theta} \frac{R_{\text {in }}^{2}}{R^{4}} \frac{j_{0}}{\rho}-\frac{j_{0}}{\rho}\left[1-\left(\frac{r}{R_{\text {in }}}\right)^{2}\right]^{-1 / 2+\theta / \pi} & \left(r<R_{\text {in }}\right), \\
\frac{4 \pi\left(R^{2}-r^{2}\right)}{\pi+2 \theta} \frac{R_{\text {in }}^{2}}{R^{4}} \frac{j_{0}}{\rho} & \left(r \geq R_{\text {in }}\right),\end{cases} \\
& Q= \begin{cases}-\frac{j_{0}}{\rho} \frac{\pi R_{\mathrm{in}}^{2}}{(\pi+2 \theta) R^{4}}\left(R^{2}-r^{2}\right)^{2}+\frac{j_{0}}{\rho} \frac{\pi R_{\mathrm{in}}^{2}}{\pi+2 \theta}\left(1-\frac{r^{2}}{R_{\mathrm{in}}^{2}}\right)^{1 / 2+\theta / \pi} & \left(r<R_{\mathrm{in}}\right), \\
-\frac{j_{0}}{\rho} \frac{\pi R_{\mathrm{in}}^{2}}{(\pi+2 \theta) R^{4}}\left(R^{2}-r^{2}\right)^{2} & \left(r \geq R_{\mathrm{in}}\right),\end{cases} \\
& Q=-\frac{j_{0}}{\rho} \frac{\pi R_{\text {in }}^{2}}{(\pi+2 \theta) R^{4}}\left(R^{2}-r^{2}\right)^{2} \leq 0\left(\text { when } r \geq R_{\text {in }}\right) .
\end{aligned}
$$

Finally, the height-averaged radial velocity $\bar{u}(r, t)$ can be derived:

$\bar{u}(r, t)=\frac{Q}{r h}= \begin{cases}-\frac{j_{0}}{\rho} \frac{\pi R_{\text {in }}^{2}}{(\pi+2 \theta) R^{3}} \frac{2\left(R^{2}-r^{2}\right)}{\theta r}+\frac{j_{0}}{\rho} \frac{\pi R_{\text {in }}^{2}}{\pi+2 \theta} \frac{2 R}{\theta\left(R^{2}-r^{2}\right) r}\left(1-\frac{r^{2}}{R_{\text {in }}^{2}}\right)^{1 / 2+\theta / \pi} & \left(r<R_{\text {in }}\right), \\ -\frac{j_{0}}{\rho} \frac{\pi R_{\text {in }}^{2}}{(\pi+2 \theta) R^{3}} \frac{2\left(R^{2}-r^{2}\right)}{\theta r} & \left(r \geq R_{\text {in }}\right) .\end{cases}$

The employed $\mu \mathrm{PIV}$ measures the velocity field at a distance of $2 \mu \mathrm{m}$ above the glass surface, which hence differs from the height-averaged velocity $\bar{u}$. We use the theory proposed by Marin et al [21] with the thin-film approximation 
to obtain the description of the velocity field. Given the low Reynolds number (low velocity) in the droplet, the inertial terms in the Navier-Stokes equation can be neglected:

$$
\frac{d p}{d r}=\mu \frac{\partial^{2} u}{\partial z^{2}},
$$

with $p$ the pressure, $\mu$ the viscosity, and $u$ the radial velocity. By solving Eq. (5.16), the velocity profile is given by

$$
u(r, z)= \begin{cases}\frac{1}{\mu} \frac{d p}{d r}\left(\frac{1}{2} z^{2}-h(r, t) z\right) & \left(r<R_{\mathrm{in}}\right), \\ \frac{1}{\mu} \frac{d p}{d r}\left(\frac{1}{2} z^{2}-\frac{1}{2} h(r, t) z\right) & \left(r \geq R_{\mathrm{in}}\right),\end{cases}
$$

where we used the boundary conditions $u(r, 0)=0$ (no slip) and $\left.\frac{\partial u}{\partial z}\right|_{z=h(r, t)}=0$ (no shear stress on the liquid-air interface) for the case $r<R_{\text {in }}$, and $u(r, 0)=0$ (no slip) and $u(r, h)=0$ (no slip on the liquid-oil interface) for the case $r>R_{\text {in }}$. Take the height average of Eq. (5.17), we obtain

$$
\bar{u}(r, t)= \begin{cases}-\frac{1}{3 \mu} \frac{d p}{d r} h^{2}(r, t) & \left(r<R_{\text {in }}\right), \\ -\frac{1}{12 \mu} \frac{d p}{d r} h^{2}(r, t) & \left(r \geq R_{\text {in }}\right),\end{cases}
$$

hence

$$
u(r, z, t)= \begin{cases}\frac{3}{h^{2}(r, t)} \bar{u}(r, t)\left(h(r, t) z-\frac{1}{2} z^{2}\right) & \left(r<R_{\text {in }}\right), \\ \frac{6}{h^{2}(r, t)} \bar{u}(r, t)\left(h(r, t) z-z^{2}\right) & \left(r \geq R_{\text {in }}\right),\end{cases}
$$

with $h$ given by Eq. (5.5), $\theta$ by Eq. (5.10) and $\bar{u}$ by Eq. (5.18). We took $R-r=80 \mu \mathrm{m}, z=2 \mu \mathrm{m}$ and $t_{e}=300 \mathrm{~s}$ the droplet's lifetime determined from the experiment.

We plot the theoretical radial velocity against the relative radial position. We use the same set of parameters as above, $R_{\text {in }}=0.8 R$ and $z=2 \mu \mathrm{m}$. From Fig. 5.9(a), we can see that the radial velocity orientates towards outside, increasing with the relative radial position. When it comes close to the position $r=R_{\text {in }}$, the velocity dramatically decreases and changes direction to be inward. After crossing the position $r=R_{\text {in }}$, the flow orientates towards inside and decreases with the radial position. From Fig. 5.9(b), the intensity of flow increases with decreasing contact angle $\theta$, which is consistent with the "rush-hour" behavior. The strong outward flow at the radial position close to $r=R_{\text {in }}$ explains the increase of the radial velocity in the early stage of "rushhour" [Fig. 3(f) in the main text]. In the experiment, the flow-reversal does not always perfectly occur at $r=R_{\text {in }}$. This is also due to the detachment of 
the contact line. When the droplet becomes very flat due to the evaporation, the contact line starts detaching from one side and the contact area shrinks towards another side. Because of this, the liquid intends to flow towards the pinning side. This manner also causes the uneven distribution of the particles in the very late stage of the droplet's lifetime. 


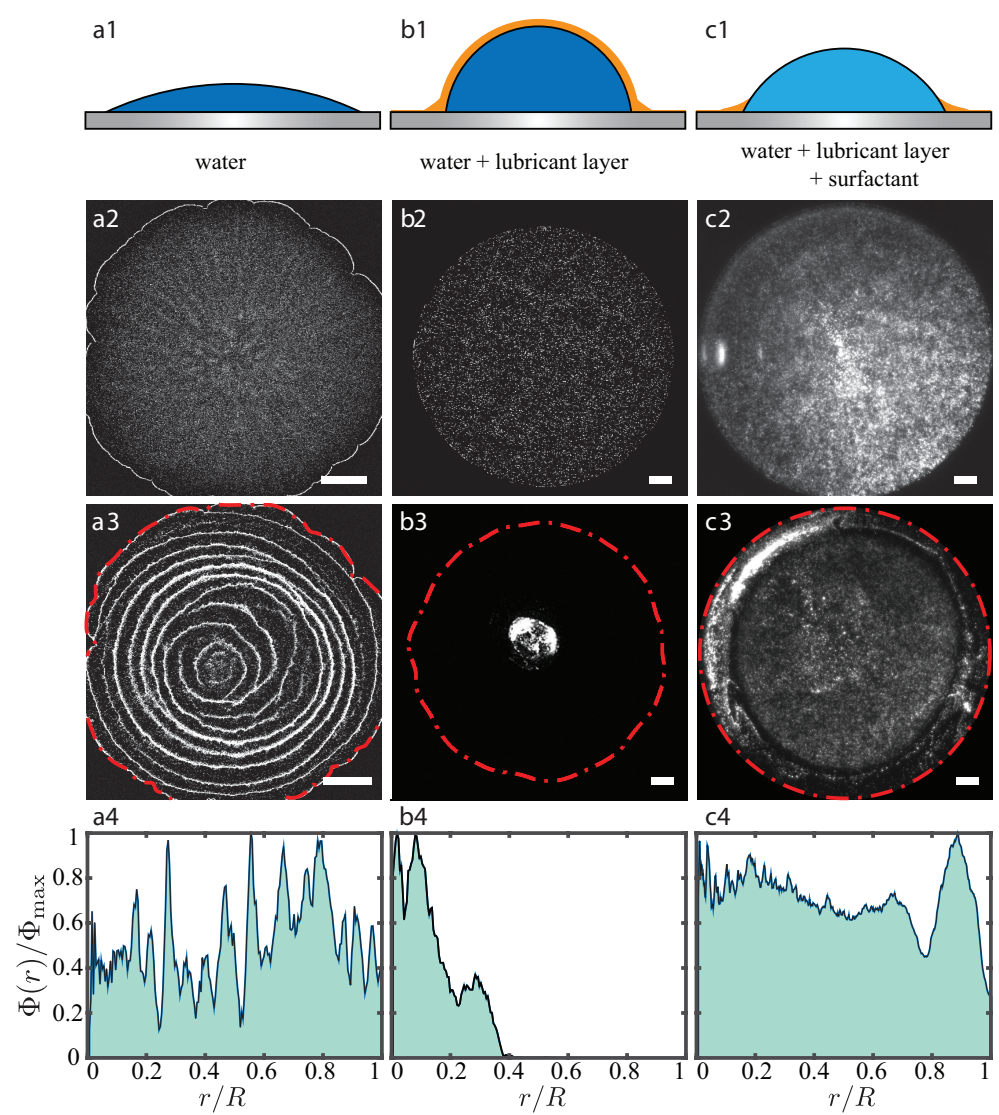

Figure 5.4: (a1-c1) Schematics of the droplets on the substrates under different conditions: (left) a colloidal water droplet on the glass slide, (middle) a colloidal water droplet on the oil-wetted surface, and (right) a colloidal water droplet seeded with surfactant SDS at concentration of $2 \mathrm{CMC}$ on the oilwetted surface. (a2-c2) The particle distribution after the deposition of each droplet in a1, b1 and c1, respectively. (a3-c3) Comparison of the final deposition patterns after full evaporation of the droplets in three cases; the red dashed lines indicate the original contact line positions. (a4-c4) Deposition profiles along the radius $r$ from the center to the original droplet edge are plotted for each of the images in $\mathrm{a} 3, \mathrm{~b} 3$ and $\mathrm{c} 3$, respectively. $R$ is the average radius of the original contact area. The intensity profile $\Phi(r)$ is normalized with the maximum of $\Phi(r)$ where $\Phi(r)=(1 / 2 \pi) \int_{0}^{2 \pi} I(r, \theta) \mathrm{d} \theta$ and $I(r, \theta)$ is the local light intensity. 


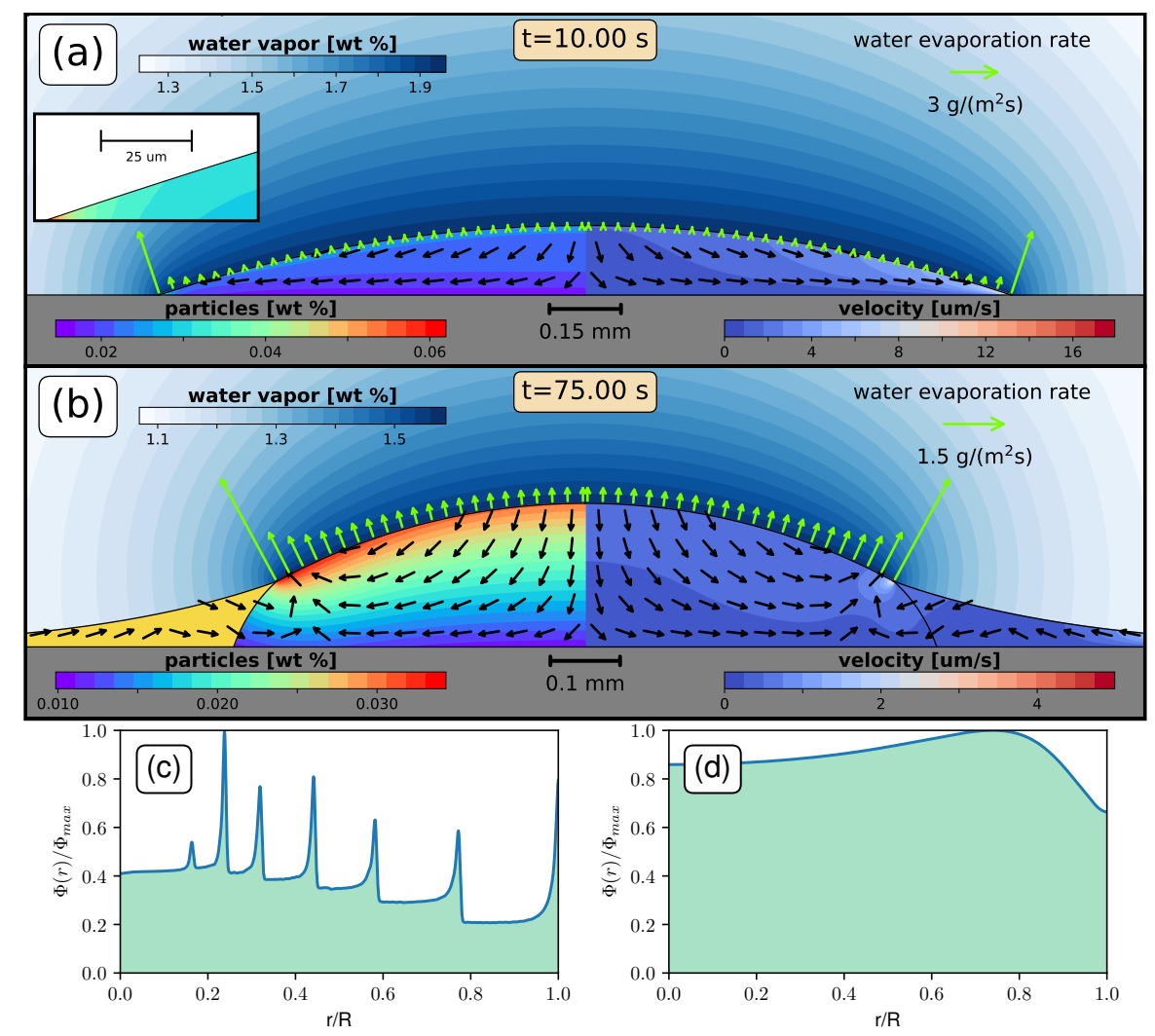

Figure 5.5: Numerical simulation of a water droplet on a glass substrate (a) and a water droplet with reduced surface tension on an oil-wetted substrate (b). The images show the vapor concentration in the gas phase and the particle concentration (left) as well as the velocity (right) and the evaporation rate at the interface. (c) Final deposition pattern of the stick-slide contact line dynamics from simulation (a). (d) Almost uniform final deposition pattern from simulation (b) as a consequence of the suppression of the coffee-stain effect via the oil film. Movies of the simulations can be found in the supplementary information. 


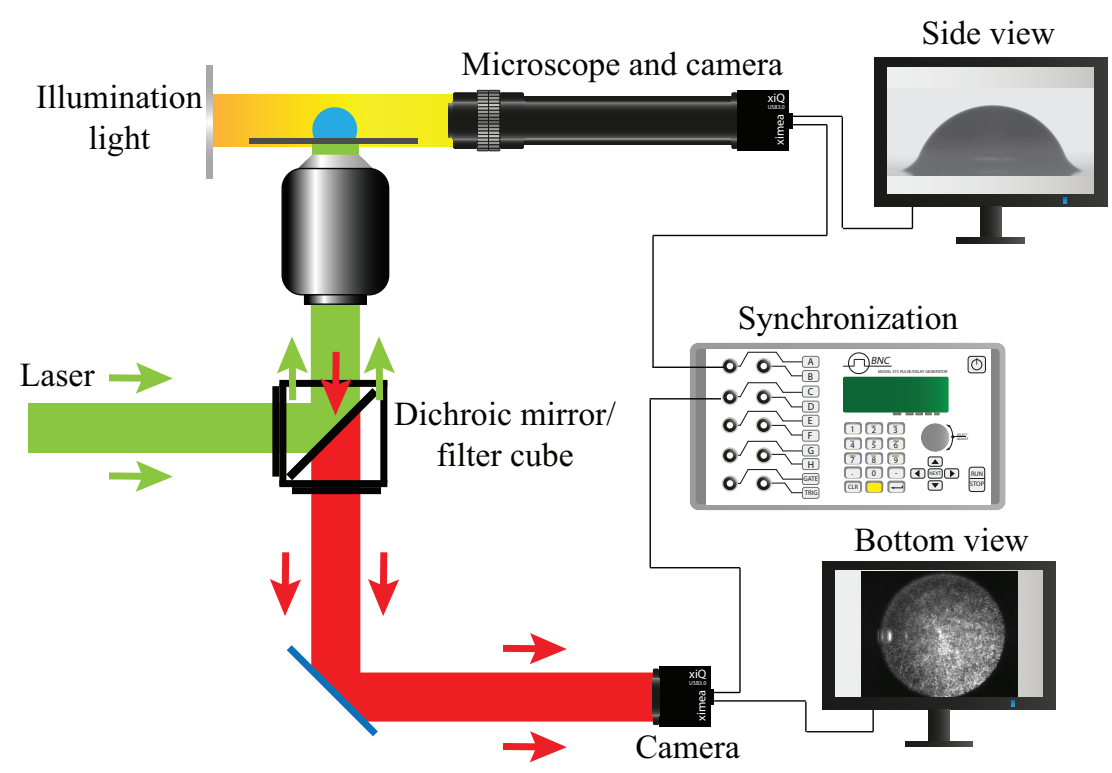

Figure 5.6: Schematic of the experimental setup employed for the simultaneous side- and bottom-view measurements during droplet evaporation. Two synchronized cameras were utilized to obtain the geometrical parameters (side view) and particle velocity (bottom view) at every time instant. 


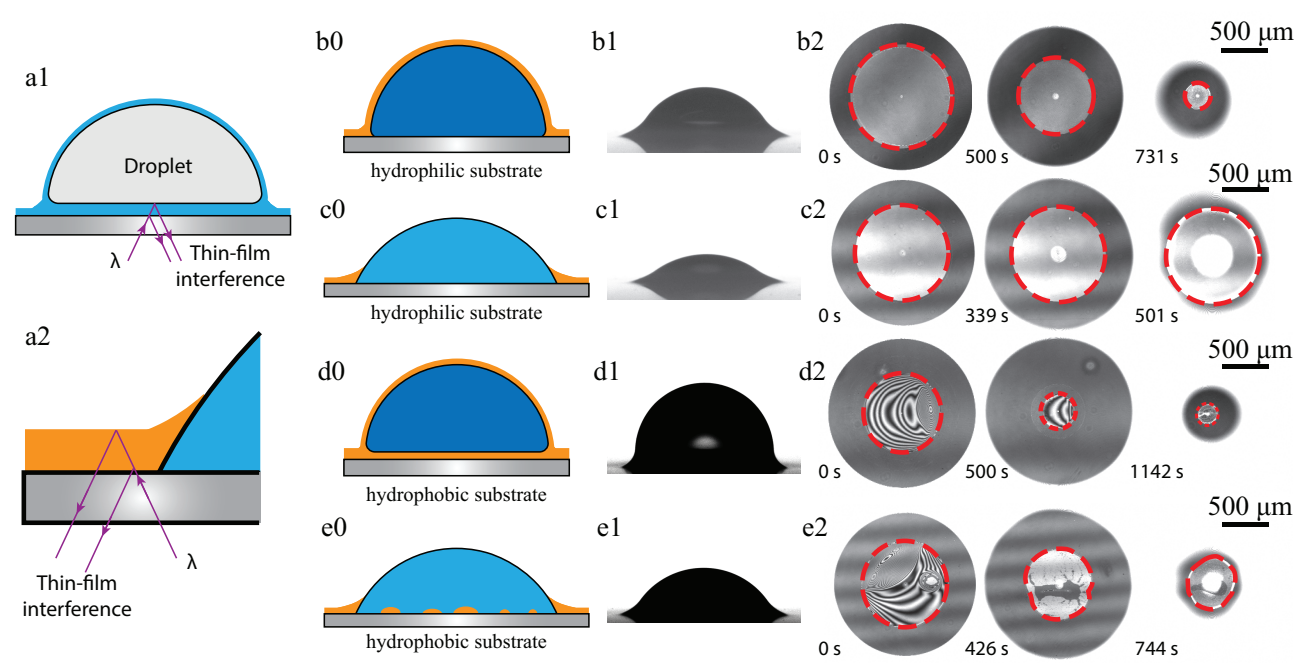

Figure 5.7: (a) Illustration of thin-film interferometry focused on surface of substrate (a1) and upper surface of the thin film (a2). (b0-e0) Schematic representing the wetting states of four cases: b0, lubricant film is completely displaced from beneath the droplet; $\mathrm{c} 0$, stable lubricant film beneath the droplet; d0, lubricant film is completely displaced from beneath the droplet; e0, lubricant film is unstable beneath a droplet and forms discrete pockets. The substrate is hydrophilic in $\mathrm{b} 0, \mathrm{c} 0$ and is hydrophobic in $\mathrm{d} 0, \mathrm{e} 0$. The droplet is water in b0,d0 and added with surfactant in c0,e0. (b1-e1) The side view for each sate in (b0-e0) at the initial moment. (b2-e2) The interferometry figures for each case during the lifetime: the focal plane is exactly on the upper surface of the substrate. b2, no fringes are observed and the contact line is unpinned. c2, no fringes are observed and the contact line is pinned. d2, fringes are observed and the contact line is moving. The droplet also slowly slides off. e2, fringes are observed in the early stage. Due to the rupture, the lubricant film is gradually displaced by the droplet and forms pockets in the center. The contact line is unpinned during the drying process. 


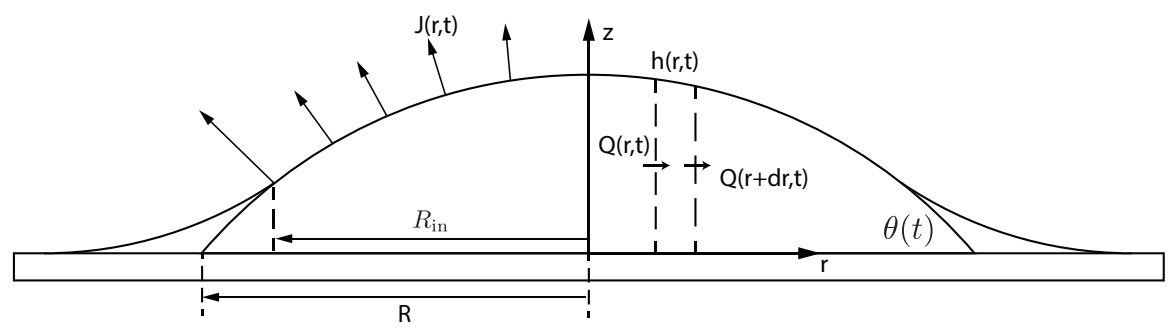

Figure 5.8: Schematics of an axisymmetric evaporating droplet, described in a cylindrical coordinate system $(r, z)$.
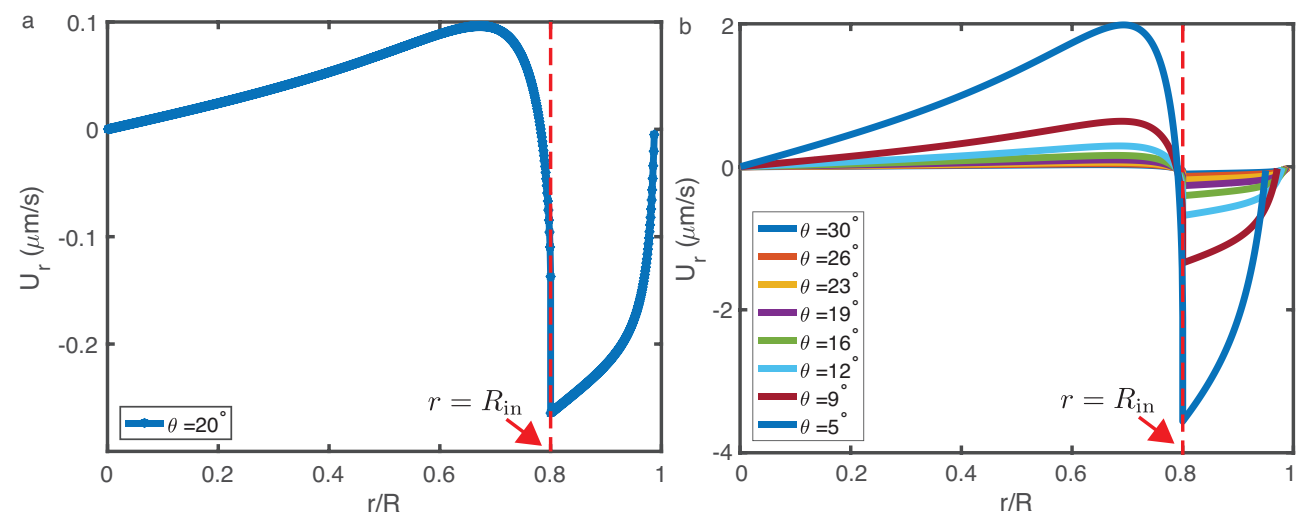

Figure 5.9: Theoretical radial velocity versus relative radial position based on Eq. (5.19). (a) The theoretical radial velocity profile for contact angle $\theta=20^{\circ}$. (b) Theoretical radial velocity profiles for a series of contact angle ranging from $5^{\circ}$ to $30^{\circ}$. 


\section{Conclusions and Outlook}

When a multicomponent droplet evaporates, the selective evaporation makes the system out of equilibrium, i.e., the preferential evaporation of more volatile liquid components causes concentration gradients, which results in surface tension gradients or density gradients. In this thesis, we have studied several multicomponent droplet systems, focusing on the evaporation behavior, flow structures and subsequent particle deposition. From a fundamental perspective, one of the simplest system has been used as the physical model: a single sessile droplet that evaporates under ambient conditions.

\section{Conclusions}

In Chapter 1, we begin with a binary liquid system consisting of 1,2-hexanediol and water, in which only water evaporates in to the atmospheric environment. It is observed that segregation within the binary droplet, that is triggered by the selective evaporation during the drying process. It is the first experimental evidence to show that segregation may occur in a fully miscible binary system. This surprising and counterintuitive finding reveals a completely new mechanism of segregation. The small surface tension differences cannot drive a strong enough Marangoni flow on the surface to induce a high enough convection within the droplet to obtain perfect mixing. Therefore a locally high concentration of 1,2-hexanediol accumulates near the contact line of the droplet, leading to segregation. Our finding is consistent with a parallel but independent study on drying of a binary droplet. Kim \& Stone [49] monitored the distribution of a certain type of particles which preferentially dissolves in one component. They also conclude that the evaporation of one component in the binary system can be so fast that it can lead to segregation.

In Chapter 2, we study another binary system of submillimeter-sized droplet by replacing 1,2-hexanediol with glycerol, which is also non-volatile in ambient 
conditions. We measure the flow field in both sessile and pendant glycerolwater droplets at submillimeter size. The $\mu \mathrm{PIV}$ measurement on the flow structure near the substrate surprisingly shows an opposite radial flow direction for each orientation. This observation clearly indicates that gravitational effects triggered by density gradients due to selective evaporation can play a dominant role in controlling the flow in evaporating multicomponent droplets, even at small Bond numbers. We introduce the Archimedes number Ar as the control parameter to predict the presence of gravity-controlled flow. With this criterion, we ignore the Marangoni effect which normally is the leading force in controlling the flow structure at such small scale. The complete picture of the competition between gravity and Marangoni effect is complicated, as it is a combination of many competing physical effects: 1 . the density difference between the two liquids; 2 . the difference in volatility; 3 . the diffusivity between the two liquids; 4 . the viscosity; 5 . the surface tension difference; 6 . the geometrical configuration, etc. We investigated the detailed picture in a follow-up paper by using the tool of numerical simulations. Here, we argued that in a relatively viscous droplet $(\operatorname{Re} \ll 1$, e.g., our glycerol-water droplet), Marangoni force barely affects the bulk flow inside the droplet, but only drives the surface flow near the liquid-air interface. In these cases, viscosity plays the role of damping, resisting the flow driven by the density gradient. Therefore, Ar can be a good indicator as a criterion for the dominance of gravity-driven flow.

In Chapter 3, we gradually increase the complexity of the system by adding a small amount of silicone oil into the binary mixture of 1,2-hexanediol and water. With the help of silicone oil, 1,2-hexanediol is extracted from the mixture causing an instantaneous segregation in the evaporating sessile droplet. Segregated fluid appears, i.e., separated 1,2-hexanediol rises up in the form of plumes from the edge to the apex of the droplet. By orientating the droplet upside down, the absence of the plumes demonstrates that the instability is controlled by gravity, as a so-called Rayleigh-Taylor instability. We have shown that it is a more extreme case than those described in Chapter 1 and 2: Became of hindrance of the strong evaporation near the contact line by segregation of the non-volatile 1,2-hexanediol, the Marangoni effect can be significantly suppressed, and it thus allows buoyancy force to play a dominant role in the flow structure.

Conventionally, Marangoni flow is considered as the dominant effect in drying drops at small scales. We have shown that the natural convection controlled by gravitational effects can also play a dominant role. In the first three chap- 
ters, the droplets display a competition between Marangoni flow and natural convection, namely "Marangoni vs. Rayleigh". In the first case, the gravitydriven flow is negligible but only Marangoni flow is dominant. In the second case, the interfacial flow driven by the surface tension is limited to a region near the droplet surface whereas the bulk flow is natural-convection-controlled by the density gradient. In the third case, the Marangoni effect is significantly suppressed and thereby, a Rayleigh-Taylor instability is generated by density gradients that appear on the droplet surface.

In Chapter 4, we study a surfactant-laden glycerol-water droplet system. We observe the crystallization of sodium dodecyl sulfate (SOS) induced by the selective evaporation of water. This system resembles an "ouzo-like" system, i.e., the depletion of water reduces the solubility of SDS in the system, eventually resulting in a segregation of SDS and the formation of crystals. We tested the applicability of a 2-dimensional model expanded from the JMAK model to describe the kinetic behavior of crystallization, which shows a good predictive value. This work may inspire the community to use surfactants in multicomponent system with greater care.

In Chapter 5, we study the evaporation of particle-laden droplets on lubricated surfaces. It is demonstrated that the interfacial energy between the droplet, the lubricated film, and the solid substrate determines the wetting state, and moreover, controls the flow structure. We show that by tuning the surface energy of the droplets through the addition of surfactants, the contact line dynamics can be manipulated such that either the coffee-stain effect can be suppressed to obtain a uniform deposit, or a size-controlled "coffee-eye" pattern can be formed. This work offers a robust method to achieve a controlled particle deposition.

In this thesis, we systematically study several multicomponent droplet systems evaporating in ambient environment. Our work progresses the fundamental physical understanding of such multicomponent systems. Moreover, we discuss the relevance of the physicochemical hydrodynamics for many important applications involving droplet evaporation, including inkjet-printing, surface coating, surface patterning and particle-assembly.

\section{Future directions}

This thesis has addressed a number of topics related to the evaporation of multicomponent droplets. Many issues still remain unexplored and open for research. 
In Chapter 1, we observe the segregation in a fully miscible binary mixture triggered by the selective evaporation. Though we analyzed the underlying mechanisms from a hydrodynamics perspective, an investigation on a molecular-level is missing. How does the local depletion of water molecules break the equilibrium of the system? Why do the 1,2-hexanediol micelles remain unstable and aggregate to form large structures? In Chapter 3, the segregation can be significantly accelerated by seeding a minute amount of silicone oil. How do the oil-water emulsions in the ternary system trigger the early extraction of 1,2-hexanediol? To answer all these questions, investigations at a micro-scopic level are required.

Chapter 2 and Chapter 3 mainly discuss the gravitational effects in evaporating microdroplets, which are normally ignored in such undersized systems dominated by surface tension. While we have shown that the suppression of the Marangoni effect allows the density gradient to drive flow at a relative long timescale. It will be interesting to further explore the instability in such a configuration (a spherical cap), either theoretically or experimentally. By varying the size of the droplet and other properties, e.g., viscosity, we can characterize the control parameters which determine the onset and the optimal wavelength of the instability.

In Chapter 5, we explore a new way to achieve uniform particle deposition by letting droplets evaporate on lubricated surfaces. We note that our method is efficient to suppress the "coffee-stain" effect until the very late stage of the droplet's lifetime. How to avoid the "rush-hour" behavior in the very end still remains the biggest challenge. In the future, we plan to focus on controlling the contact line movement at the final stage of the drying process.

In recent years, the evaporation of multicomponent droplets attracted significant attention as a result of their ubiquitous role in either nature or technology [131]. Our findings clearly show many unexpected phenomena in such evaporating systems. This particularly holds for inkjet printing, as nearly all inks contain various components with different volatility. These variations of the composition ratio caused by the selective evaporation of more volatile components may lead to the segregation of less volatile components in the form of liquid phase separation [64] or crystallization [119]. Our study may rise the awareness to utilize the interactions between different components as to control evaporation lifetime, internal flows and final particle deposition in multicomponent ink droplets.

This not only holds for the "second phase" of inkjet printing, namely the evaporation phase of ink drops on paper or other substrates; but also on the 
nozzle-plate of the inkjet channel during the "first phase", which is known as the jetting phase. The selective evaporation of ink droplets at the nozzle exit may cause major issues for the jetting [131]: 1. the selective evaporation of the liquid meniscus in the nozzle within the time interval between two jetting cycles results in changes of the material properties of the remaining ink; 2 . the Marangoni flow in the liquid on the nozzle plate originating from the surface tension gradients caused by selective evaporation can lead to transport of dirt particle into nozzle. These are major issues leading to misprints or even failure. Our study may offer a new perspective to suppress such selective evaporation by phase separation of non-volatile components, and hence to maintain the stability of the material properties of the ink, leading to an improved printing quality. 


\section{References}

[1] H. W. Morse, "Evaporation from the surface of a sphere", Proc. Am. Acad. Arts Sci. 45, 361 (1910).

[2] I. Langmuir., "The evaporation of small spheres", Phys. Rev. 12, 368370 (1918).

[3] P. S. Epstein and M. S. Plesset, "On the stability of gas bubbles in liquid-gas solutions", J. Chem. Phys. 18, 1505-1509 (1950).

[4] H. Gelderblom, A. G. Marin, H. Nair, A. van Houselt, L. Lefferts, J. H. Snoeijer, and D. Lohse, "How water droplets evaporate on a superhydrophobic substrate", Phys. Rev. E 83, 026306 (2011).

[5] Y. O. Popov, "Evaporative deposition patterns: Spatial dimensions of the deposit", Phys. Rev. E. 71, 036313 (2005).

[6] R. G. Picknett and R. Bexon, "The evaporation of sessile or pendant drops in still air", J. Colloid Interface Sci. 61, 336-350 (1977).

[7] E. Dietrich, E. S. Kooij, X. Zhang, H. J. W. Zandvliet, and D. Lohse, "Stick-jump mode in surface droplet dissolution", Langmuir 31, 46964703 (2015).

[8] D. Debuisson, A. Merlen, V. Senez, and S. Arscott, "Stick jump (sj) evaporation of strongly pinned nanoliter volume sessile water droplets on quick drying, micropatterned surfaces", Langmuir 32, 2679-2686 (2016).

[9] D. Lohse and X. Zhang, "Surface nanobubble and surface nanodroplets", Rev. Mod. Phys. 87, 981-1035 (2015).

[10] C. Marcolli and T. Peter, "Water activity in polyol/water systems: new unifac parameterization", Atmos. Chem. Phys. 5, 1545-1555 (2005). 
[11] S. Chu and A. Prosperetti, "Dissolution and growth of a multicomponent drop in an immiscible liquid", J. Fluid Mech. 798, 787-811 (2016).

[12] R. D. Deegan, O. Bakajin, T. F. Dupont, G. Huber, S. R. Nagel, and T. A. Witten, "Capillary flow as the cause of ring stains from dried liquid drops", Nature 389, 827-829 (1997).

[13] J. Thomson, "On certain curious motions observable on the surfaces of wine and other alcoholic liquours", Philosophical Magazine 10, 330-333 (1855).

[14] C. Marangoni, "Sull' espansione delle gocce di un liquido gallegianti sulla superficie di altro liquido (on the expansion of a drop of liquid floating in the surface of another liquid)", Tipographia dei fratelli (1865).

[15] H. Hu and R. G. Larson, "Evaporation of a sessile droplet on a substrate", J. Phys. Chem. B. 106, 1334 (2002).

[16] A. M. Cazabat and G. Guéna, "Evaporation of macroscopic sessile droplets", Soft Matter 6, 2591-2612 (2010).

[17] N. Shahidzadeh-Bonn, S. Rafai, A. Azouni, and D. Bonn, "Evaporating droplets", J. Fluid Mech. 549, 307-313 (2006).

[18] W. D. Ristenpart, P. G. Kim, C. Domingues, J. Wan, and H. A. Stone, "Influence of substrate conductivity on circulation reversal in evaporating drops", Phys. Rev. Lett. 99, 234502 (2007).

[19] J. A. Lim, W. H. Lee, H. S. Lee, J. H. Lee, Y. D. Park, and K. Cho, "Self-organization of ink-jet-printed triisopropylsilylethynyl pentacene via evaporation-induced flows in a drying droplet", Adv. Functional Mat. 18, 229-234 (2008).

[20] F. Schönfeld, K. H. Graf, S. Hardt, and H.-J. Butt, "Evaporation dynamics of sessile liquid drops in still air with constant contact radius", Int. J. Heat Mass Transfer 51, 3696-3699 (2008).

[21] A. G. Marin, H. Gelderblom, D. Lohse, and J. H. Snoeijer, "Order-todisorder transition in ring-shaped colloidal stains", Phys. Rev. Lett. 107, 085502 (2011).

[22] D. Brutin, B. Sobac, B. Loquet, and J. Sampol, "Pattern formation in drying drops of blood", J. Fluid Mech. 667, 85-95 (2011). 
[23] R. Ledesma-Aguilar, D. Vella, and J. M. Yeomans, "Lattice-Boltzmann simulations of droplet evaporation", Soft Matter 10, 8267-8275 (2014).

[24] H. Tan, C. Diddens, P. Lv, J. G. M. Kuerten, X. Zhang, and D. Lohse, "Evaporation-triggered microdroplet nucleation and the four life phases of an evaporating ouzo drop", Proc. Natl. Acad. Sci. U.S.A. 113, 86428647 (2016).

[25] J. Park and J. Moon, "Control of colloidal particle deposit patterns within picoliter droplets ejected by ink-jet printing", Langmuir 22, 35063513 (2006).

[26] M. Kuang, L. Wang, and Y. Song, "Controllable printing droplets for high-resolution patterns", Adv. Mater. 26, 6950-6958 (2014).

[27] J. Jing, J. Reed, J. Huang, X. Hu, V. Clarke, J. Edington, D. Housman, T. S. Anantharaman, E. J. Huff, and B. Mishra, "Automated high resolution optical mapping using arrayed, fluid-fixed DNA molecules", Proc. Natl. Acad. Sci. U.S.A. 95, 8046-8051 (1998).

[28] T. A. H. Nguyen, S. R. Biggs, and A. V. Nguyen, "Manipulating colloidal residue deposit from drying droplets: Air/liquid interface capture competes with coffee-ring effect", Chem. Eng. Sci. 167, 78-87 (2017).

[29] K. Sefiane, L. Tadrist, and M. Douglas, "Experimental study of evaporating water-ethanol mixture sessile drop: influence of concentration", Int. J. Heat Mass Transfer 46, 4527-4534 (2003).

[30] K. Sefiane, S. David, and M. E. R. Shanahan, "Wetting and evaporation of binary mixture drops", J. Phys. Chem. B 112, 11317-11323 (2008).

[31] H. Kim, F. Boulogne, E. Um, I. Jacobi, E. Button, and H. A. Stone, "Controlled uniform coating from the interplay of Marangoni flows and surface-adsorbed macromolecules", Phys. Rev. Lett. 116, 124501 (2016).

[32] C. Diddens, H. Tan, P. Lv, M. Versluis, J. Kuerten, X. Zhang, and D. Lohse, "Evaporating pure, binary and ternary droplets: thermal effects and axial symmetry breaking", J. Fluid Mech. 823, 470-497 (2017).

[33] P. Löbl, M. Huppertz, and D. Mergel, "Nucleation and growth in TiO2 films prepared by sputtering and evaporation", Thin Solid Films 251, 72-79 (1994). 
[34] N. Wang, Y. Tang, Y. Zhang, C. S. Lee, and S. T. Lee, "Nucleation and growth of Si nanowires from silicon oxide", Phys. Rev. B 58, R16024 (1998).

[35] N. P. Rao and P. H. McMurry, "Nucleation and growth of aerosol in chemically reacting systems: A theoretical study of the near-collisioncontrolled regime", Aerosol. Sci. Technol. 11, 120-132 (1989).

[36] A. A. Hyman, C. A. Weber, and F. Jülicher, "Liquid-liquid phase separation in biology", Annu. Rev. Cell Dev. Biol. 30, 39-58 (2014).

[37] C. A. Kennedy, S. N. MacMillan, M. J. McAlduff, and D. G. Marangoni, "The interaction of isomeric hexanediols with sodium dodecyl sulfate and dodecyltrimethylammonium bromide micelles", Colloid Polym. Sci. 279, 1-7 (2001).

[38] G. D'Arrigo, R. Giordano, and J. Teixeira, "Small-angle neutron scattering studies of aqueous solutions of short-chain amphiphiles", Eur. Phys. J. E 10, 135-142 (2003).

[39] S. M. Hajji, M. B. Errahmani, R. Coudert, R. R. Durand, A. Cao, and E. Taillandier, "A comparative study of 1, 2-hexanediol and 1, 2, 3 -octanetriol in aqueous solutions by different physical techniques", J. Phys. Chem. 93, 4819-4824 (1989).

[40] M. Frindi, B. Michels, and R. Zana, "Ultrasonic absorption studies of surfactant exchange between micelles and bulk phase in aqueous micellar solutions of nonionic surfactants with short alkyl chains. 1. 1, 2-hexanediol and 1, 2, 3-octanetriol", J. Phys. Chem. 95, 4832-4837 (1991).

[41] N. K. Székely, L. Almásy, A. Rădulescu, and L. Rosta, "Small-angle neutron scattering study of aqueous solutions of pentanediol and hexanediol", J. Appl. Crystallogr. 40, 307-311 (2007).

[42] C. Romero, M. S. Páez, J. A. Miranda, D. J. Hernández, and L. E. Oviedo, "Effect of temperature on the surface tension of diluted aqueous solutions of 1,2-hexanediol, 1,5-hexanediol, 1,6-hexanediol and 2,5hexanediol", Fluid Phase Equilib. 258, 67 - 72 (2007).

[43] E. Dietrich, S. Wildeman, C. Visser, K. Hofhuis, E. S. Kooij, H. J. W. Zandvliet, and D. Lohse, "Role of natural convection in the dissolution of sessile droplets", J. Fluid Mech. 794, 45-67 (2016). 
[44] A. Marin, R. Liepelt, M. Rossi, and C. J. Kähler, "Surfactant-driven flow transitions in evaporating droplets", Soft Matter 12, 1593-1600 (2016).

[45] C. Diddens, J. Kuerten, C. van der Geld, and H. Wijshoff, "Modeling the evaporation of sessile multi-component droplets", J. Colloid Interf. Sci. 487, 426-436 (2017).

[46] C. Diddens, "Detailed finite element method modeling of evaporating multi-component droplets", J. Comput. Phys. 340, 670-687 (2017).

[47] H. Tan, C. Diddens, M. Versluis, H.-J. Butt, D. Lohse, and X. Zhang, "Self-wrapping of an ouzo drop induced by evaporation on a superamphiphobic surface", Soft Matter 13, 2749-2759 (2017).

[48] R. P. Sear, "Quantitative studies of crystal nucleation at constant supersaturation: experimental data and models", Cryst. Eng. Comm. 16, 6506-6522 (2014).

[49] H. Kim and H. Stone, "Direct measurement of selective evaporation of binary mixtures by dissolving materials", J. Fluid Mech. 850, 769 (2018).

[50] A. van der Bos, M.-J. van der Meulen, T. Driessen, M. van den Berg, H. Reinten, H. Wijshoff, M. Versluis, and D. Lohse, "Velocity profile inside piezoacoustic inkjet droplets in flight: Comparison between experiment and numerical simulation", Phys. Rev. Applied 1, 014004 (2014).

[51] W. Thielicke and E. J. Stamhuis, "Pivlab towards user-friendly, affordable and accurate digital particle image velocimetry in matlab", Journal of Open Research Software (2014).

[52] D. Garcia, ""a fast all-in-one method for automated post-processing of piv data"”, Exp. Fluids 50, 1247-1259 (2011).

[53] P. Jarosiewicz, G. Czechowski, and Jadzyn, "The viscous properties of diols. v. 1,2-hexanediol in water and butanol solutions", Z. Naturforsch. A 59, 559-562 (2004).

[54] W. Hayduk and B. S. Minhas, "Correlations for prediction of molecular diffusivities in liquids", The Canadian Journal of Chemical Engineering 60, 295-299 (1982). 
[55] A. Zuend, C. Marcolli, B. P. Luo, and T. Peter, "A thermodynamic model of mixed organic-inorganic aerosols to predict activity coefficients", Atmos. Chem. Phys. 8, 4559-4593 (2008).

[56] A. Zuend, C. Marcolli, A. M. Booth, D. M. Lienhard, V. Soonsin, U. K. Krieger, D. O. Topping, G. McFiggans, T. Peter, and J. H. Seinfeld, "New and extended parameterization of the thermodynamic model AIOMFAC: calculation of activity coefficients for organic-inorganic mixtures containing carboxyl, hydroxyl, carbonyl, ether, ester, alkenyl, alkyl, and aromatic functional groups", Atmos. Chem. Phys. 11, 91559206 (2011).

[57] C. Marcolli and T. Peter, "Water activity in polyol/water systems: new UNIFAC parameterization", Atmos. Chem. Phys. 5, 1545-1555 (2005).

[58] Y. L. Kong, I. A. Tamargo, H. Kim, B. N. Johnson, M. K. Gupta, T. Koh, H. Chin, D. A. Steingart, B. P. Rand, and M. C. McAlpine, "3d printed quantum dot light-emitting diodes", Nano Lett. 14, 7017-7023 (2014).

[59] H. Hu and R. G. Larson, "Marangoni effect reverses coffee-ring depositions", J. Phys. Chem. B 110, 7090-7094 (2006).

[60] D. Tam, V. von Arnim, G. H. McKinley, and A. E. Hosoi, "Marangoni convection in droplets on superhydrophobic surfaces", J. Fluid Mech. 624, 101 (2009).

[61] J. R. E. Christy, Y. Hamamoto, and K. Sefiane, "Flow transition within an evaporating binary mixture sessile drop", Phys. Rev. Lett. 106, 205701 (2011).

[62] R. Bennacer and K. Sefiane, "Vortices, dissipation and flow transition in volatile binary drops", J. Fluid Mech. 749, 649-665 (2014).

[63] H. Tan, C. Diddens, M. Versluis, H.-J. Butt, D. Lohse, and X. Zhang, "Self-wrapping of an ouzo drop induced by evaporation on a superamphiphobic surface", Soft Matter 13, 2749-2759 (2017).

[64] Y. Li, P. Lv, C. Diddens, H. Tan, H. Wijshoff, M. Versluis, and D. Lohse, "Evaporation-triggered segregation of sessile binary droplet", Phys. Rev. Lett. 120, 224501 (2018). 
[65] D. H. Shin, S. H. Lee, J.-Y. Jung, and Y. J. Y., "Evaporating characteristics of sessile droplet on hydrophobic and hydrophilic surfaces", Microelectron. Eng. 86, 1350 - 1353 (2009).

[66] R. Savino and R. Monti, "Buoyancy and surface-tension-driven convection in hanging-drop protein crystallizer", J. Cryst. Growth 165, $308-$ 318 (1996).

[67] K. H. Kang, H. C. Lim, H. W. Lee, and S. J. Lee, "Evaporation-induced saline Rayleigh convection inside a colloidal droplet", Phys. Fluids 25, 042001 (2013).

[68] T. K. Pradhan and P. K. Panigrahi, "Influence of an adjacent droplet on fluid convection inside an evaporating droplet of binary mixture", Colloids Surf. A 500, $154-165$ (2016).

[69] T. K. Pradhan and P. K. Panigrahi, "Evaporation induced natural convection inside a droplet of aqueous solution placed on a superhydrophobic surface", Colloids Surf. A 530, 1 - 12 (2017).

[70] A. Kumar and D. K. Mandal, "Oscillatory circulation inside evaporating methanol-water drops", International Journal of Multiphase Flow 102, $130-137$ (2018).

[71] "Soap and detergent association", Technical Report of the Soap and Detergent Association 212 (1990).

[72] R. Sousa, "Use of glycerol, polyols and other protein structure stabilizing agents in protein crystallization", Acta Cryst. D 51, 271-277 (1995).

[73] "Physical properties of glycerine and its solutions", Glycerine Producers Association, New York (1963).

[74] S. Shin, I. Jacobi, and H. A. Stone, "Bénard-Marangoni instability driven by moisture absorption", EPL 113, 24002 (2016).

[75] N. Xue, S. Khodaparast, L. Zhu, J. K. Nunes, H. Kim, and H. A. Stone, "Laboratory layered latte", Nat. Commun. 8, 1960 (2017).

[76] H. Yu, Z. Lu, D. Lohse, and X. Zhang, "Gravitational effect on the formation of surface nanodroplets", Langmuir 31, 12628 (2015). 
[77] Y. Tanaka, K. Ohta, H. Kubota, and T. Makita, "Viscosity of aqueous solutions of 1,2-ethanediol and 1,2-propanediol under high pressures", Int. J. Thermophys 9, 511 (1988).

[78] K. Nakanishi, T. Matsumoto, and M. Hayatsu, "Surface tension of aqueous solutions of some glycols", J. Chem. Eng. Data 16, 44-45 (1971).

[79] A. M. J. Edwards, P. S. Atkinson, C. S. Cheung, H. Liang, D. J. Fairhurst, and F. F. Ouali, "Density-driven flows in evaporating binary liquid droplets", Phys. Rev. Lett. 121, 184501 (2018).

[80] S. Peng, C. Xu, T. C. Hughes, and X. Zhang, "From nanodroplets by the ouzo effect to interfacial nanolenses", Langmuir 30, 12270-12277 (2014).

[81] J. Park and J. Moon, "Control of colloidal particle deposit patterns within picoliter droplets ejected by ink-jet printing", Langmuir 22, 35063513 (2006).

[82] M. Kuang, L. Wang, and Y. Song, "Controllable printing droplets for high-resolution patterns", Adv. Mater. 26, 6950-6958 (2014).

[83] K. L. Chong, Y. Li, C. S. Ng, R. Verzicco, and D. Lohse, "Convectiondominated dissolution for single and multiple immersed sessile droplets", J. Fluid Mech. 892, A21 (2020).

[84] G. Brenn, L. Deviprasath, F. Durst, and C. Fink, "Evaporation of acoustically levitated multi-component liquid droplets", Int. J. Heat Mass Transfer. 50, 5073-5086 (2007).

[85] S. Karpitschka, F. Liebig, and H. Riegler, "Marangoni contraction of evaporating sessile droplets of binary mixtures", Langmuir 33, 46824687 (2017).

[86] A. Marin, S. Karpitschka, D. Noguera-Marín, M. A. Cabrerizo-Vílchez, M. Rossi, C. J. Kähler, and M. A. Rodríguez Valverde, "Solutal marangoni flow as the cause of ring stains from drying salty colloidal drops", Phys. Rev. Fluids 4, 041601 (2019).

[87] X. Zhong and F. Duan, "Flow regime and deposition pattern of evaporating binary mixture droplet suspended with particles", Eur. Phys. J. E 2 (2016). 
[88] Y. Li, C. Diddens, P. Lv, H. Wijshoff, M. Versluis, and D. Lohse, "Gravitational effect in evaporating binary microdroplets", Phys. Rev. Lett. 122, 114501 (2019).

[89] H. Tan, S. Wooh, H.-J. Butt, X. Zhang, and D. Lohse, "Microdroplet nucleation by dissolution of a multicomponent drop in a host liquid", Nat. Commun. 10, 478 (2019).

[90] E. Dietrich, M. Rump, P. Lv, E. S. Kooij, H. J. Zandvliet, and D. Lohse, "Segregation in dissolving binary-component sessile droplets", J. Fluid Mech. 812, 349-369 (2017).

[91] H. Tan, C. Diddens, A. A. Mohammed, J. Li, M. Versluis, X. Zhang, and D. Lohse, "Microdroplet nucleation by dissolution of a multicomponent drop in a host liquid", J. Fluid Mech. 870, 217 (2019).

[92] S. Karpitschka, "The value of a fading tracer", J. Fluid Mech. 856, 1 (2018).

[93] R. Alany, T. Rades, S. Agatonovic-Kustrin, N. Davies, and I. Tucker, "Effects of alcohols and diols on the phase behaviour of quaternary systems", Int. J. Pharm. 196, 141 - 145 (2000).

[94] L. Rayleigh, "Investigation of the character of the equilibrium of an incompressible heavy fluid of variable density", Proc. Lond. Math. Soc. s1-14, 170 (1882).

[95] G. Taylor, "The instability of liquid surfaces when accelerated in a direction perpendicular to their planes", Proc. R. Soc. Lond. 201, 192 (1950).

[96] D. H. Sharp, "An overview of Rayleigh-Taylor instability", Int. J. Pharm. 12, 3 - 18 (1984).

[97] D. H. Olson and J. W. Jacobs, "Experimental study of rayleigh-taylor instability with a complex initial perturbation", Phys. Fluids 21, 034103 (2009).

[98] R. P. Berklaar, E. Dietrich, G. A. M. Kip, E. S. Kooij, H. J. W. Zandvliet, and D. Lohse, "Exposing nanobubble-like objects to a degassed environment", Soft Matter 10, 4947 (2014). 
[99] F. Schellenberger, J. Xie, N. Encinas, A. Hardy, M. Klapper, P. Papadopoulos, H.-J. Butt, and D. Vollmer, "Direct observation of drops on slippery lubricant-infused surfaces", Soft Matter 11, 7617-7626 (2015).

[100] A. Gao, J. Liu, L. Ye, C. Schönecker, M. Kappl, H.-J. Butt, and W. Steffen, "Control of droplet evaporation on oil-coated surfaces for the synthesis of asymmetric supraparticles", Langmuir 35, 14042-14048 (2019).

[101] M. A. Hack, M. Costalonga, T. Segers, S. Karpitschka, H. Wijshoff, and J. H. Snoeijer, "Printing wet-on-wet: Attraction and repulsion of drops on a viscous film", Appl. Phys. Lett. 113, 183701 (2018).

[102] D. Daniel, J. V. I. Timonen, R. Li, S. J. Velling, and J. Aizenberg, "Oleoplaning droplets on lubricated surfaces", Nat. Phys. 13, 1020 (2017).

[103] B. Sobac and D. Brutin, "Triple-line behavior and wettability controlled by nanocoated substrates: Influence on sessile drop evaporation", Langmuir 27, 14999-15007 (2011).

[104] J. Stauber, S. Wilson, B. Duffy, and K. Sefiane, "On the lifetimes of evaporating droplets", J. Fluid Mech. 744, R2 (2014).

[105] J. Stauber, S. Wilson, B. Duffy, and K. Sefiane, "On the lifetimes of evaporating droplets with related initial and receding contact angles", Phys. Fluids 27, 122101 (2015).

[106] T. A. H. Nguyen and A. V. Nguyen, "Increased evaporation kinetics of sessile droplets by using nanoparticles", Langmuir 28, 16725-16728 (2012).

[107] B. Sobac and D. Brutin, "Desiccation of a sessile drop of blood: Cracks, folds formation and delamination", Colloids Surf. A: Physicochem. Eng. Asp. 448, $34-44$ (2014).

[108] R. Battino, "Volume changes on mixing for binary mixtures of liquids", Chem. Rev. 71, 5 (1971).

[109] W. Sempels, R. De Dier, H. Mizuno, J. Hofkens, and J. Vermant, "Autoproduction of biosurfactants reverses the coffee ring effect in a bacterial system", Nat. Commun 4 (2013).

[110] A. Marin, R. Liepelt, M. Rossi, and C. J. Kahler, "Surfactant-driven flow transitions in evaporating droplets", Soft Matter 12, 1593-1600 (2016). 
[111] W. Kwieciński, T. Segers, S. van der Werf, A. van Houselt, D. Lohse, H. J. W. Zandvliet, and S. Kooij, "Evaporation of dilute sodium dodecyl sulfate droplets on a hydrophobic substrate", Langmuir 35, 10453-10460 (2019).

[112] T. Still, P. J. Yunker, and A. G. Yodh, "Surfactant-induced marangoni eddies alter the coffee-rings of evaporating colloidal drops", Langmuir 28, 4984-4988 (2012).

[113] J. Piret, A. Desormeaux, and M. Bergeron, "Sodium lauryl sulfate, a microbicide effective against enveloped and nonenveloped viruses", Curr. Drug Targets 3, 17-30 (2002).

[114] A. Kumar, G. Bhattacharjee, B. D. Kulkarni, and R. Kumar, "Role of surfactants in promoting gas hydrate formation", Industrial \& Engineering Chemistry Research 54, 12217-12232 (2015).

[115] N. Choudhary, V. R. Hande, S. Roy, S. Chakrabarty, and R. Kumar, "Effect of sodium dodecyl sulfate surfactant on methane hydrate formation: A molecular dynamics study", The Journal of Physical Chemistry B 122, 6536-6542 (2018).

[116] L. Smith, A. Duncan, G. Thomson, K. Roberts, D. Machin, and G. McLeod, "Crystallisation of sodium dodecyl sulphate from aqueous solution: phase identification, crystal morphology, surface chemistry and kinetic interface roughening", Journal of Crystal Growth 263, 480 - 490 (2004).

[117] E. Summerton, G. Zimbitas, M. Britton, and S. Bakalis, "Crystallisation of sodium dodecyl sulfate and the corresponding effect of 1-dodecanol addition", Journal of Crystal Growth 455, 111 - 116 (2016).

[118] S. A. Vitale and J. L. Katz, "Liquid droplet dispersions formed by homogeneous liquid-liquid nucleation: "the ouzo effect"", Langmuir 19, 4105-4110 (2003).

[119] A. Mailleur, C. Pirat, O. Pierre-Louis, and J. Colombani, "Hollow rims from water drop evaporation on salt substrates", Phys. Rev. Lett. 121, 214501 (2018).

[120] M. T. Geballe, A. G. Skillman, A. Nicholls, J. P. Guthrie, and P. J. Taylor, "The sampl2 blind prediction challenge: introduction and overview", Journal of Computer-Aided Molecular Design 24, 259-279 (2010). 
[121] F.-M. Raoult, "Loi générale des tensions de vapeur des dissolvants", Comptes rendus 104, 1430-1433 (1887).

[122] S. Chu and A. Prosperetti, "Dissolution and growth of a multicomponent drop in an immiscible liquid", J. Fluid Mech. 798, 787-811 (2016).

[123] M. Avrami, "Kinetics of phase change. i general theory", J. Chem. Phys 7, 1103 (1939).

[124] M. Avrami, "Kinetics of phase change. ii transformation-time relations for random distribution of nuclei", J. Chem. Phys 8, 212 (1940).

[125] M. Avrami, "Granulation, phase change, and microstructure kinetics of phase change iii", J. Chem. Phys 9, 177 (1941).

[126] W. Johnson and R. Mehl, "Reaction kinetics in processes of nucleation and growth", Trans. AIME 135, 416 (1939).

[127] A. Kolmogorov, "A statistical theory for the recrystallization of metals", Isz. Akad. Nauk SSR. Ser. Mat. 3, 355 (1937).

[128] M. C. Weinberg, "Surface nucleated transformation kinetics in 2- and 3dimensional finite systems", J. Non-Cryst. Solids 134, 116 - 122 (1991).

[129] M. C. Weinberg, D. P. Birnie, and V. A. Shneidman, "Crystallization kinetics and the jmak equation", J. Non-Cryst. Solids 219, 89 - 99 (1997).

[130] H. Manikantan and T. M. Squires, "Surfactant dynamics: hidden variables controlling fluid flows", J. Fluid Mech. 892, P1 (2020).

[131] D. Lohse and X. Zhang, "Physicochemical hydrodynamics of droplets out of equilibrium: A perspective review", Nat. Rev. Phys. in press (2020).

[132] C. C. Ruiz, L. Díaz-López, and J. Aguiar, "Micellization of sodium dodecyl sulfate in glycerol aqueous mixtures", J. Disper. Sci. and Technol. 29, 266-273 (2008).

[133] B. M. Weon and J. H. Je, "Self-pinning by colloids confined at a contact line", Phys. Rev. Lett. 110, 028303 (2013). 
[134] E. L. Talbot, H. N. Yow, L. Yang, A. Berson, S. R. Biggs, and C. D. Bain, "Printing small dots from large drops", ACS Appl. Mater. Interfaces 7, $3782(2015)$.

[135] W. Han and Z. Lin, "Learning from coffee rings: Ordered structures enabled by controlled evaporative self-assembly", Angew. Chem. Int. Ed. 51, 1534 (2012).

[136] T. P. Bigioni, X.-M. Lin, T. T. Nguyen, E. I. Corwin, T. A. Witten, and H. M. Jaeger, "Kinetically driven self assembly of highly ordered nanoparticle monolayers", Nat. Mater. 5, 265 (2006).

[137] B. M. Weon and J. H. Je, "Capillary force repels coffee-ring effect", Phys. Rev. E 82, 015305 (2010).

[138] V. X. Nguyen and K. J. Stebe, "Patterning of small particles by a surfactant-enhanced marangoni-bénard instability", Phys. Rev. Lett. 88, 164501 (2002).

[139] Y. Li, C. Lv, Z. Li, D. Quéré, and Q. Zheng, "From coffee rings to coffee eyes", Soft Matter 11, 4669-4673 (2015).

[140] P. J. Yunker, T. Still, M. A. Lohr, and A. G. Yodh, "Suppression of the coffee-ring effect by shape-dependent capillary interactions", Nature 476, 308 (2011).

[141] L. Shmuylovich, A. Q. Shen, and H. A. Stone, "Surface morphology of drying latex films: multiple ring formation", Langmuir 18, 3441-3445 (2002).

[142] A. Askounis, K. Sefiane, V. Koutsos, and M. E. Shanahan, "The effect of evaporation kinetics on nanoparticle structuring within contact line deposits of volatile drops", Colloids Surf. A 441, 855 - 866 (2014).

[143] Y. Ooi, I. Hanasaki, D. Mizumura, and Y. Matsuda, "Suppressing the coffee-ring effect of colloidal droplets by dispersed cellulose nanofibers", Science and Technology of Advanced Materials 18, 316-324 (2017).

[144] S. Mishra, K. L. Barton, A. G. Alleyne, P. M. Ferreira, and J. A. Rogers, "High-speed and drop-on-demand printing with a pulsed electrohydrodynamic jet", J. Micromech. Microeng. 20, 095026 (2010). 
[145] A. P. Mouat, C. E. Wood, J. E. Pye, and J. C. Burton, "Tuning contact line dynamics and deposition patterns in volatile liquid mixtures", Phys. Rev. Lett. 124, 064502 (2020).

[146] S. Y. Lee, H. Kim, S.-H. Kim, and H. A. Stone, "Uniform coating of selfassembled noniridescent colloidal nanostructures using the marangoni effect and polymers", Phys. Rev. Applied 10, 054003 (2018).

[147] A. Mailleur, C. Pirat, O. Pierre-Louis, and J. Colombani, "Hollow rims from water drop evaporation on salt substrates", Phys. Rev. Lett. 121, 214501 (2018).

[148] C. Diddens, Y. Li, and D. Lohse, "Competing marangoni and rayleigh convection in evaporating binary droplets", Submitted to J. Fluid Mech. (2020).

[149] T.-S. Wong, S. H. Kang, S. K. Y. Tang, E. J. Smythe, B. D. Hatton, A. Grinthal, and J. Aizenberg, "Bioinspired self-repairing slippery surfaces with pressure-stable omniphobicity", Nature 477, 443 (2011).

[150] J. B. Boreyko, G. Polizos, P. G. Datskos, S. A. Sarles, and C. P. Collier, "Air-stable droplet interface bilayers on oil-infused surfaces", Proc. Natl. Acad. Sci. U.S.A. (2014).

[151] A. Carlson, P. Kim, G. Amberg, and H. A. Stone, "Short and long time drop dynamics on lubricated substrates", EPL 104 (2013).

[152] F. Schellenberger, J. Xie, N. Encinas, A. Hardy, M. Klapper, P. Papadopoulos, H.-J. Butt, and D. Vollmer, "Direct observation of drops on slippery lubricant-infused surfaces", Soft Matter 11, 7617-7626 (2015).

[153] J. D. Smith, R. Dhiman, S. Anand, E. Reza-Garduno, R. E. Cohen, G. H. McKinley, and K. K. Varanasi, "Droplet mobility on lubricantimpregnated surfaces", Soft Matter 9, 1772-1780 (2013).

[154] C. Semprebon, G. McHale, and H. Kusumaatmaja, "Apparent contact angle and contact angle hysteresis on liquid infused surfaces", Soft Matter 13, 101-110 (2017).

[155] D. Daniel, J. V. I. Timonen, R. Li, S. J. Velling, M. J. Kreder, A. Tetreault, and J. Aizenberg, "Origins of extreme liquid repellency on structured, flat, and lubricated hydrophobic surfaces", Phys. Rev. Lett. 120, 244503 (2018). 
[156] J. H. Guan, G. G. Wells, B. Xu, G. McHale, D. Wood, J. Martin, and S. Stuart-Cole, "Evaporation of sessile droplets on slippery liquid-infused porous surfaces (slips)", Langmuir 31, 11781-11789 (2015).

[157] K. J. Mysels, "Surface tension of solutions of pure sodium dodecyl sulfate", Langmuir 2, 423 (1986).

[158] A. G. Kanellopoulos and M. J. Owen, "Adsorption of sodium dodecyl sulphate at the silicone fluid/water interface", Trans. Faraday Soc. 67, 3127 (1971).

[159] P. Mukerjee and K. J. Mysels, "Critical micelle concentration of aqueous surfactant systems", Washington, DC: US. Government Printing (1971).

[160] G. McHale, B. V. Orme, G. G. Wells, and R. Ledesma-Aguilar, "Apparent contact angles on lubricant-impregnated surfaces/slips: From superhydrophobicity to electrowetting", Langmuir 35, 4197-4204 (2019).

[161] H. Tan, S. Wooh, H.-J. Butt, X. Zhang, and D. Lohse, "Porous superparticle assembly through self-lubricated evaporating colloidal ouzo drops", Nat. Commun. 10, 478 (2019).

[162] S. Das, A. Dey, G. Reddy, and D. Sarma, "Suppression of the coffee-ring effect and evaporation-driven disorder to order transition in colloidal droplets", J. Phys. Chem. Lett. 8, 4704-4709 (2017).

[163] F. Reizman, "Optical thickness measurement of thin transparent films on silicon", J. Appl. Phys. 36, 3804-3807 (1965).

[164] M. Heil and A. L. Hazel, "oomph-lib - an object-oriented multi-physics finite-element library. fluid-structure interaction, lecture notes on computational science and engineering", 19-49 (2006).

[165] Y. Li, C. Diddens, A. Prosperetti, K. L. Chong, X. Zhang, and D. Lohse, "Bouncing oil droplet in a stratified liquid and its sudden death", Phys. Rev. Lett. 122, 154502 (2019).

[166] A. Gauthier, C. Diddens, R. Proville, D. Lohse, and D. van der Meer, "Self-propulsion of inverse leidenfrost drops on a cryogenic bath", Proc. Natl Acad. Sci. USA 116, 1174-1179 (2019).

[167] H. R. Baker, W. D. Bascom, and C. R. Singleterry, "The adhesion of ice to lubricated surfaces", J. Colloid Sci. 17, 477 - 491 (1962). 


\section{Summary}

Multicomponent droplets are ubiquitous in nature and technology. It is of great importance to understand their evaporation process. In this thesis, we have studied several multicomponent droplet systems, while focusing on the evaporation behavior, flow structures and subsequent particle deposition. We have applied multiple experimental techniques, theoretical analysis and numerical simulations to systematically study the evaporation process from different aspects.

We first studied the evaporation of 1,2-hexanediol-water binary droplets (Chapter 1). In this work, we observed and studied phase segregation of an evaporating sessile binary droplet, consisting of a miscible mixture of water and a surfactant-like liquid (1,2-hexanediol). The phase segregation (i.e., demixing) leads to a reduced water evaporation rate of the droplet. Eventually the evaporation process ceases due to shielding of the water by the non-volatile 1,2-hexanediol. Visualizations of the flow field by particle image velocimetry and numerical simulations reveal that the timescale of water evaporation at the droplet rim is faster than that of the Marangoni flow. This originates from the surface tension difference between water and 1,2-hexanediol, eventually leading to segregation. This insight has provided the first demonstration that the phase separation can be triggered by the selective evaporation in a miscible binary droplet.

In Chapter 2, the flow in an evaporating glycerol-water binary sub-millimeter droplet with Bond number Bo $\ll 1$ is studied, both experimentally and numerically. First, we measure the flow fields near the substrate by micro-PIV for both sessile and pendant droplets during the evaporation process. Surprisingly, they show opposite radial flow directions - inward and outward, respectively. These observations clearly reveals that in spite of the small droplet size, gravitational effects play a crucial role in controlling the flow fields in the evaporating droplets. We theoretically analyze that this gravity-driven effect is triggered by the lower volatility of glycerol which leads to a preferential 
evaporation of water then the local concentration difference of the two components leads to a density gradient that drives the convective flow. We show that the Archimedes number, Ar, is the nondimensional control parameter for the occurrence of the observed gravitational effects. We confirm our hypothesis by experimentally comparing two evaporating microdroplet systems, namely a glycerol-water droplet and a 1,2-propanediol-water droplet. We obtain different Ar, larger or smaller than unity by varying a series of droplet heights, which corresponds to cases with or without gravitational effects, respectively. Finally, we simulate the process numerically, finding good agreement with the experimental results and again confirming our interpretation.

As a next step, we added $0.5 \mathrm{wt} \%$ silicone oil into the 1,2-hexanediol-water binary solution (Chapter 3 ). This minute silicone oil concentration dramatically modifies the evaporation process as it triggers an early extraction of the 1,2-hexanediol from the mixture. Surprisingly, we observe that the segregation of 1,2-hexanediol forms plumes, rising up from the rim of the sessile droplet towards the apex during the droplet evaporation. By orientating the droplet upside down, i.e., by studying a pendant droplet, the absence of the plumes indicates that the flow structure is induced by buoyancy, which drives a Rayleigh-Taylor instability (i.e., driven by density differences \& gravitational acceleration). From $\mu$ PIV measurement, we further prove that the segregation of the non-volatile component (1,2-hexanediol) hinders the evaporation near the contact line, which leads to a suppression of the Marangoni flow in this region. Hence, on long time scales, gravitational effects play the dominant role in the flow structure, rather than Marangoni flows. We compare the measurement of the evaporation rate with the diffusion model of Popov [5], coupled with Raoult's law and the activity coefficient. This comparison indeed confirms that the silicone-oil-triggered segregation of the non-volatile 1,2-hexanediol significantly delays the evaporation. With an extended diffusion model, in which the influence of the segregation has been implemented, the evaporation can be well described.

In Chapter 4, we observed and studied the crystallization of sodium dodecyl sulfate (SDS) within an evaporating glycerol-water mixture droplet. The crystallization is induced by the preferential evaporation of water, which decreases the solubility of SDS in the mixture. As a consequence, the crystals shield the droplet surface and cease the evaporation. The universality of the evaporation characteristics for a range of droplet sizes is revealed by applying a diffusion model, extended by Raoult's law. To describe the nucleation and growth of the crystals, we employ the 2-dimensional crystallization model of Weinberg, 
J. Non-Cryst. Solids, 1991, 134, 116. The results of this model compare favorably to our experimental results. Our findings may inspire the community to reconsider the role of high concentration of surfactants in multicomponent evaporation system.

In Chapters 1-4, we have seen that different segregation behaviors can be triggered by the selective evaporation. In Chapter 5 , we moved to the particle deposition by the evaporation of suspension droplets, which is the underlying mechanism in many surface coating and surface patterning applications. However, the uniformity of the final deposit suffers from the coffee-stain effect caused by contact line pinning. Here, we showed that control over particle deposition can be achieved through droplet evaporation on oil-wetted surfaces. We demonstrated by flow visualization, theory, and numerics that the final deposit of the particles is governed by the coupling of the flow field in the evaporating droplet, the movement of its contact line, and the wetting state of the thin film surrounding the droplet. We showed that the dynamics of the contact line can be tuned through the addition of a surfactant, thereby controlling the surface energies, which then leads to control over the final particle deposit. We also obtained an analytical expression for the radial velocity profile which reflects hindrance of the evaporation at the rim of the droplet by the non-volatile oil meniscus. It prevents flow towards the contact line, thus suppressing the coffee-stain effect. Finally, we confirmed our physical interpretation by numerical simulations that are in qualitative agreement with the experiment. 


\section{Samenvatting}

Druppels bestaande uit meerdere componenten zijn alomtegenwoordig in de natuur en de techniek. Het is daarom belangrijk om hun verdampingsproces goed te begrijpen. In dit proefschrift hebben we een aantal systemen met druppels die uit meerdere componenten bestaan onderzocht. Daarbij hebben we ons met name toegelegd op hun verdampingsgedrag, hun stromingsstructuren, en de daaropvolgende deeltjesdepositie. Wij hebben meerdere experimentele technieken, theoretische methoden, en numerieke simulaties gebruikt om verscheidene aspecten van het verdampingsprocess systematisch te bestuderen. Als eerste hebben we de verdamping van 1,2-hexaandiol-water binaire druppels onderzocht (Hoofdstuk 1). In dit werk hebben we fasescheiding geobserveerd en onderzocht in een verdampende binaire sessiele druppel, bestaande uit een mengbare combinatie van water met een vloeistof met oppervlakte-actieve eigenschappen. De fasescheiding (d.w.z., ontmenging) resulteert in een tragere verdamping van water. Uiteindelijk stopt de verdamping van het water door de afschermende werking van het niet-vluchtige 1,2-hexaandiol. Visualisaties van het stromingveld door velocimetrie met deeltjes en numerieke simulaties tonen aan dat de tijdschaal van de verdamping van water bij de rand van de druppel sneller is dan die van de Marangoni-stroming, die ontstaat door het verschil in oppervlaktespanning van water en 1,2-hexaandiol, wat uiteindelijk leidt tot fasescheiding. Dit werk heeft voor het eerst aangetoond dat fasescheiding kan worden veroorzaakt door selectieve verdamping in een mengbare binaire druppel.

In Hoofdstuk 2, bestuderen we de stroming in een verdampende glycerol-water binaire submillimeter druppel met Bondgetal Bo $\ll 1$ middels experimenten en numerieke simulaties. Eerst meten we de stromingsvelden dichtbij het substraat met micro-PIV voor zowel sessiele als hangende druppels tijdens het verdampingsproces. Dit toont dat zij verrassend genoeg tegengestelde radiale stromingsrichtingen tonen - respectievelijk naar binnen en naar buiten. Deze observatie laat zien dat zwaartekrachtseffecten zelfs voor kleine druppels een 
cruciale rol spelen in het controleren van de stromingsvelden van verdampende druppels. We laten aan de hand van theoretische argumenten zien dat dit door zwaartekracht aangedreven proces wordt veroorzaakt door de lagere vluchtigheid van glycerol, wat leidt tot een preferentiële verdamping van water, waarna het lokale concentratieverschil van de twee componenten leidt tot een dichtheidsgradiënt die de convectieve stroming aandrijft. We laten zien dat het Archimedesgetal Ar de relevante niet-dimensionale parameter is voor het optreden van zwaartekrachtseffecten. We bevestigen onze hypothese door experimenteel twee verdampende microdruppelsystemen te vergelijken, namelijk een glycerol-waterdruppel en een 1,2-propaandiol-waterdruppel. We verkrijgen verschillende Ar door de druppelhoogte te variëren. Deze Archimedesgetallen, groter en kleiner dan een één, komen overeen met de situatie met respectievelijk zonder zwaartekrachtseffecten. Ten slotte vinden we een goede overeenkomst tussen numerieke simulaties en de experimentele resultaten, en bevestigen we opnieuw onze interpretatie.

Als volgende stap hebben we 0,5 gew.\% siliconenolie toegevoegd aan de 1,2hexaandiol-water binaire oplossing (Hoodstuk 3). Deze zeer kleine concentratie siliconenolie wijzigt het verdampingsproces dramatisch omdat het een vroege fasescheiding van 1,2-hexaandiol veroorzaakt. Verrassend genoeg zien we dat de fasescheiding van 1,2-hexaandiol pluimen vormt, die tijdens de verdamping van de druppel opstijgen vanaf de rand van de sessiele druppel naar de top van de druppel. Door de druppel ondersteboven te oriënteren, d.w.z. door een hangende druppel te bestuderen, geeft de afwezigheid van de pluimen aan dat de stromingstructuur wordt veroorzaakt door het drijfvermogen, wat een Rayleigh-Taylor-instabiliteit veroorzaakt (d.w.z. aangedreven door dichtheidsverschillen en zwaartekrachtversnelling) Middels $\mu \mathrm{PIV}$ metingen bewijzen we verder dat de fasescheiding van de niet-vluchtige component (1,2-hexaandiol) de verdamping nabij de contactlijn belemmert, wat leidt tot een onderdrukking van de Marangoni-stroming in dit gebied. Zwaartekrachteffecten hebben daarom op lange tijdsschalen de dominante rol in de stromingsstructuur, in plaats van Marangoni-stroming. We vergelijken de meting van de verdampingssnelheid met het diffusiemodel van Popov [5], gekoppeld aan de wet van Raoult en de activiteitscofficiënt. Deze vergelijking bevestigt inderdaad dat de door siliconenolie veroorzaakte fasescheiding van het nietvluchtige 1,2-hexaandiol de verdamping aanzienlijk vertraagt. Een uitgebreid diffusiemodel, waarin de invloed van de fasescheiding is geïmplementeerd, beschrijft de verdamping goed.

In Hoofdstuk 4 hebben we de kristallisatie van natriumdodecylsulfaat (SDS) 
in een verdampende glycerol-waterdruppel geobserveerd en bestudeerd. De kristallisatie wordt veroorzaakt door de snellere verdamping van water, waardoor de oplosbaarheid van SDS in het mengsel vermindert. Als gevolg hiervan bedekken de kristallen het druppeloppervlak en stoppen ze de verdamping. De algemeenheid van de verdampingseigenschappen voor een reeks druppelgroottes wordt onthuld door een diffusiemodel toe te passen, uitgebreid door de wet van Raoult. Om de vorming en groei van de kristallen te beschrijven gebruiken we het twee-dimensionale kristallisatiemodel van Weinberg, $J$. Non-Cryst. Solids, 1991, 134, 116. Onze experimentele resultaten worden goed beschreven door dit model. Onze bevindingen kunnen de gemeenschap inspireren om de rol van hoge concentraties van oppervlakteactieve stoffen in verdampingssystemen met meerdere componenten te heroverwegen.

In Hoofdstukken 1-4 hebben we gezien dat verscheidene fasescheidingsgedragingen kunnen worden veroorzaakt door selectieve verdamping. In Hoofdstuk 5, hebben we deeltjesdepositie door de verdamping van suspensiedruppels bestudeerd. Dit is het onderliggende mechanisme van veel toepassingen die te maken hebben met het coaten van oppervlakken en het aanbrengen van patronen op oppervlakken. De gelijkmatigheid van de uiteindelijke depositie lijdt echter onder het koffievlekeffect dat wordt veroorzaakt door het vastzitten van de contactlijn. Hier tonen we aan dat controle over de deeltjesdepositie kan worden bereikt door druppelverdamping op met olie bedekte oppenvlakken. Doormiddel van stromingsvisualisatie, theoretische analyse, en numerieke simulaties laten we zien dat de uiteindelijke deeltjesdepositie wordt bepaald door de koppeling van het stromingsveld in de verdampende druppel, de beweging van de contactlijn, en de bevochtigingstoestand van de dunne laag rond de druppel. Wij laten zien dat de dynamica van de contactlijn kan worden gecontroleerd door de toevoeging van een oppervlakteactieve stof, die de oppervlakte-energieën controleert, wat vervolgens leidt tot controle over de uiteindelijke deeltjesdepositie. Wij hebben ook een analytische uitdrukking gevonden voor het radiale snelheidsprofiel dat de belemmering van de verdamping aan de rand van de druppel door de niet-vluchtige oliemeniscus beschrijft. Dit voorkomt stroming naar de contactlijn, waardoor het koffievlekeffect onderdrukt wordt. Ten slotte hebben we onze fysieke interpretatie bevestigd door numerieke simulaties die in kwalitatieve overeenstemming zijn met het experiment. 


\section{Scientific output}

\section{Publications}

- Yaxing Li, Pengyu Lv, Christian Diddens, Huanshu Tan, Herman Wijshoff, Michel Versluis, Detlef Lohse

Evaporation-triggered segregation of sessile binary droplets,

Phys. Rev. Lett. 120, 224501 (2018),

- Yaxing Li, Christian Diddens, Pengyu Lv, Herman Wijshoff, Michel Versluis, Detlef Lohse

Gravitational effect in evaporating binary microdroplets,

Phys. Rev. Lett. 122, 114501 (2019),

- Yaxing Li, Christian Diddens, Tim Segers, Herman Wijshoff, Michel Versluis, Detlef Lohse

Evaporating droplets on oil-wetted surfaces: Suppression of the coffeestain effect,

Proc. Natl. Acad. Sci. U.S.A. (2020) (In press),

- Yaxing Li, Christian Diddens, Tim Segers, Herman Wijshoff, Michel Versluis, Detlef Lohse

Rayleigh-Taylor instability by segregation in an evaporating multi-component microdroplet,

J. Fluid Mech. (2020) (In press),

- Yaxing Li, Valentin Salvator, Herman Wijshoff, Michel Versluis, Detlef Lohse

Evaporating-induced crystallization of surfactants in sessile multicomponent droplets,

Langmuir (2020) (In press), 
- Christian Diddens, Yaxing Li, Detlef Lohse

Competing Marangoni and Rayleigh convection in evaporating binary droplets,

J. Fluid Mech. (2020) (Under review).

\section{Invited and selected talks}

- Max-Planck Center Meeting (invited), Mainz, Germany, Feb. 2020

- 72nd APS DFD, Seattle, US, Nov. 2019

- Droplets 2019, UK Durham, Sep. 2019

- Lorentz center inkjet printing workshop (invited), Leiden, NL, Jul. 2019

- Burgers Symposium, Lunteren, NL, Jan. 2019

- Physics@Veldhoven, Veldhoven NL, Jan. 2019

- 71st APS DFD, Atlanta US, Nov. 2018

- 70th APS DFD, Denver US, Nov. 2017

\section{Scientific workshops \& Schools}

- Complex Fluids Summer School, DK, Aug. 2019

- Complex Fluids Summer School, UK Cambridge, Sep. 2016

- Complex Fluids Summer School, DK, Aug. 2015 


\section{Prizes \& Awards}

- Chinese Government Outstanding Self-Financed Students Abroad Award (2020)

\section{Selected student supervision}

- Bachelor student:

Hannah Brugge (Expected Oct. 2020)

- Master student:

Valentin Salvator (Apr. - Jul. 2018)

\section{Experience}

- Academic Visit to USTC, China, member of the organization committee. (Apr. 2017)

- Laboratory general supplier, Physics of Fluids group. (2017-2020)

- Teaching assistant for the Master course: "Experimental techniques in physics of fluids" for the study Applied Physics in Twente. (2017-2019)

- Teaching assistant for Physics of Bubbles course, Master course. (20162020)

- Research Internship, "Bubble entrainment in printhead", Océ Technologies B. V., Venlo, The Netherlands. (Apr. - Jul. 2016)

\section{Peer review}

- Reviewer for Journal of Fluid Mechanics (JFM), International Journal of Multiphase Flow (IJMF), and Proceedings of the National Academy of Sciences (PNAS). 


\section{Acknowledgements}

It is hard to believe that the journey is approaching the ending. Seven years ago, I got a phone call from Prof. Chao Sun from the Physics of Fluids group when I was applying for the master programme at University of Twente. Now I still believe that it is the luckiest phone-call I've ever received in my life. One year later, I started my study in Enschede. Some people say, the city where you live in your 20s leaves its own personality on you. If this is true, I wish I've learned the kindness, the enthusiasm and creativeness from this beautiful town. Looking back at this amazing journey, there are so many people I would like to thank.

First of all, I am very blessed to have the best supervisors any Ph.D could wish for: Detlef Lohse and Michel Versluis.

Dear Detlef, I still vividly remember that our first conversation was in your Turbulence course in which you encouraged me to talk to you whenever I needed. Throughout my Ph.D pursuits, I always feel your enthusiasm and support. Thank you for providing such a great atmosphere for the group and for every one of us. During one of our drivings to Venlo, you told me that the essence to be an outstanding researcher is to have a good taste on science. Since then, I always challenge myself to ask good questions. Your passion on science and your attitude on work always teach me to be curious, to cherish the value of time. I am, and will always be proud of being your student.

Dear Michel, our first essential interaction was the rehearsal of my master defence in which you helped me to polish my slides. It turned out to be the most important lesson in my career to really learn how to make a nice presentation. Since the beginning of Ph.D, you have invited me to your 2-weekly meetings. I really appreciate and greatly benefit from these meetings, in which I can share many immature ideas with you and always get constructive feedback. Thank you for letting me be your teaching assistant, which is an invaluable experience and training for my future career. 
Dear Herman, you are my third supervisor from the Océ. You always come up with so many interesting ideas for us to study. Thank you for inspiring me to think from so many different views and angles. Dear Hans and Marc, you are the supervisors of my intern. Thank you for taking care of me. It was a great experience to work in a world-class company.

Dear Chao, as I said in the beginning, it is one of the luckiest moment in my life to receive your invitation to the group. Thank you for all the advices, help and encouragement throughout my stay in the Netherlands. Without you, there is not even a start for this journey. Dear Andrea, I have to admit that discussing with you is very challenging but very inspiring! You taught me how important for a researcher to be critical and precise. Dear Jacco, if I had a chance, I would join every lecture you give. I am amazed by your sense of humor which is always full of wisdom. I appreciated and enjoyed every discussion we had. You told me that the lubrication equation is your favourite, I wish in the future, I will also have my favourite one. I'd love to thank Devaraj and Álvaro for the nice discussions and excellent courses.

Dear Pengyu and Christian, I cannot express my gratitudes to both of you. No work can be done in this thesis without you. Pengyu, you taught me everything you know when I just started my Ph.D, experimental technique, post-processing skills, writing ... You are my academic old brother, my friend and my teacher! Christian, you participated in almost every work in my thesis. Your amazing numerical simulations and stunning colour code have already became your signature. I really appreciate all the scientific discussions and random talks we had. I wish and I am sure you will become a great professor in the future!

It is a great pleasure to pay tribute to Joanita, Bas, Gert-Wim, Martin B., and Dennis G.. As Detlef always says, the group will not notice his absence, but be very sensitive to yours. Joanita, thank you for taking care of me and all of us for so many years. Almost everyone in our group is welcomed by you and your warm smile in their first day. Bas, thank you for helping me with my laptop and item ordering. Gert-Wim, thank you for sharing your memory in China with me. Dennis, thank you for arranging everything in such a nice way!

My dear officemates, dear Mengqi, Borge, Anais, Anja, Jassica, and Steven. It is so lucky for me to have such wonderful officemates like you. Mengqi, I 
really enjoy the great time we had together in the office, at my place, and in Denver. Anais, you are a rising star in the fluid dynamics. I admire the way you perform the research work. Anja, I enjoyed the futsal we played together. Jassica, I really appreciate all the conversations we had. You are a sensitive girl with a warm and big heart. Thank you for being my English teacher! You are the colour of PoF! Steven, it is such a pleasure to have you in my office. You are a wonderful teacher and an amazing friend. I wish you can achieve what you want in the near future.

My former officemates back to the old days during my master. Dear Dennis, Rodrigo, Christa, Martin, Xiumei, Leonie, Mathijs, Sebastian, Maura, Pilar, and Mirjam. Thank you for being so nice to me when I just joined the group. I still miss the dinners and parties we had. Dear Ivan, you are my true hero. You took so much care of me when I was a freshman. I still remember the every moment of us in Frankfurt. I heard that you are doing great now, I hope I can visit you sometime in your city!

My teammates in PoF United. Dear Ruben, Pieter, Mike, Mikhail, Álvaro, Nakul, Marvin, Youssef, Raymond, and Jelle. I enjoyed every single match with you guys. Dear Alexander, you are the best goal-keeper I have ever played in my life! Thank you for the coffee-breaks we had, thank you for all the warm things to comfort me whenever I was down. Dear Pieter, you are the best captain!

My inkjet printing fellas. Dear Michiel, Tim, Maaike, Yogesh, Arjan, Wojtek, and Udo. I enjoyed every trip we had to Venlo. Dear Michiel, we have shared hotel rooms for so many times at different cities, for different conferences. You are the best roommate I have ever had, and you are an amazing friend to me. I learned so much from your organized and disciplined lifestyle. Dear Tim, you are an amazing experimentalist and a great writer! I enjoyed working with you so much!

My Chinese folks in the group. Dear Yantao, Xiaojue, Huanshu, Haoran, Yanshen, Luoqin, Qi, Rui, Junyi, Xiaolai, Binglin, Guiquan, Jiaming, Songchuan, Xiaoyan, Jun, and Haitao. I am so grateful to have all of you these years in my life. Dear Yuliang and Zhenhua, I leaned so much from you. Dear Yibo and Shuai, we have a great friendship together which I will value and appreciate for the rest of my life.

My dear friends in Enschede. Dear Han, Luyao, Chongnan, Yuhan, Songbo, 
Ying, Lantian, Bingying, Danqi, lovely Sipei, Lingna, and Zhuoyun. Thank you for your company making me never feel lonely!

Of course, PoF can never be such a great group without all of the young PoFers! I couldn't remember how much I learned and gained from you these years. Dear Guillaume, I really appreciate all the generous help you offered me. "Even a french people deserves a good place to work". You deserve the best facilities to achieve your academic goal! Dear Sander, you are the best photographer I have ever met in my life! Thank you for taking me to all different places in the world by your camera! Dear Pierre, Mazi and Pallav, you are the greatest experimentalists! Dear Chong Shen, thank you for teaching me tennis! Dear Myrthe, we got to know each other since our master. You are so smart and talented. I enjoyed the travel we had in the UK. Dear Álvaro, Varghese, Vamsi and Anupam, you are the old generation. I wish you the best in the US! Utkarsh, you are the coolest Indian! I enjoyed talking all different kinds of stuff with you! Dear Mathieu, Pablo, Martin, Nathan, Peter, Diana, Robert, Minkush, Srinath, Yoan, Ricardo, Ali, Saeed, Vatsal, Farzan, Carola, Lijun, José, Marie-Jean, Maxime... Thank you for the talks, the greetings and many small things we had. I would like to thank my master supervisor Erik-Jan, you welcomed me to the group and taught me so many things. I am so blessed to have you as my supervisor. It was you to initiate my life in the PoF. I also want to thank my student Valentin, you are so smart. I am happy that our work ends up with a nice publication!

Finally, I own my most profound gratitude to my beloved family: my parents and my wife. Your love, support and trust are the only reason of me to achieve all of these!

Yaxing $\mathrm{Li}$

Enschede, June 2020 


\section{About the author}

Yaxing Li was born on December 22nd 1991 in Anhui, China. He spent the first 18 years of his life in a beautiful small city, Maanshan. He has been interested in Physics since the middle school. In 2010, he moved to Hefei where he studied Theoretical Mechanics at University of Science and Technology of China. During his Bachelor, he found his passion on fluid mechanics. In 2013, he joined the group of Prof. Jiming Yang for his Bachelor thesis. In 2014, he moved to Enschede, the Netherlands to start his Master at University of Twente. For his Master's thesis, Yaxing worked on the experimental study on the evaporation of sessile droplets, in the Physics of Fluids group. After his Master defence, he spent three months in Venlo for an internship at Canon Production Printing. He obtained his Master's diploma in 2016. In August 2016 he continued his PhD research in the same group with Prof. Detlef Lohse and Prof. Michel Versluis.

Apart from doing research, Yaxing likes to spend time with his friends, in football fields, in basketball courts, in swimming pools and on snow mountains. He is passionate on food and cooking. In the spare time, he enjoys music by playing saxophone and acoustic guitar. He also learned painting for six years in the primary school, including three-year traditional Chinese ink painting. 
